\title{
Estudios Sobre Criptógamas Colombianas III. Lista comentada de los Musgos de Colombia ${ }^{1}$
}

\author{
J. Florschütz-de WAARD ${ }^{2}$ AND P. A. FlorschÜTZ†
}

\begin{abstract}
A survey of the literature in which species of Musci are reported for Colombia, amplified by unpublished identifications of recent collections, indicates a known flora of 750 species. About 600 published names are treated as synonyms. An annotated list of the collectors is also provided, as well as notes on critical localities and itineraries, especially those of Purdie, Lindig and Wallis. Moss collections of Moritz, Wagner and Osculati are not from Colombia. Two new combinations are proposed: Campylopus pittieri Williams var. congestum (Thér.) comb. nov. and $\mathrm{C}$. pittieri var. latilimbatum (Thér.) comb. nov.
\end{abstract}

En este trabajo se presenta una lista de 750 taxa, como una contribución al conocimiento de la distribución de los musgos en Suramérica, particularmente en Colombia. La mayor parte de los datos para la preparación de ésta lista, así como gran parte de la literatura briológica correspondiente, fueron recopilados por mi esposo, el Dr. P. A. Florschütz, quien desafortunadamente falleció antes de completarla.

Existen varias listas similares para otros paises suraméricanos. Con el fin de dar una idea de la información existente, incluyo a continuación un resumen de las contribuciones más importantes, en orden cronológico. Ecuador: 781 especies, Steere, 1948 (datos adicionales: Crum, 1957; Bartram, 1955, 1964; Robinson et al., 1971, 1977). Venezuela: 626 especies, Pursell, 1973 (datos adicionales: Griffin, 1973, 1975, 1977a). Peru: 568 especies, Hegewald, 1975 (datos adicionales: Hegewald, 1977). Bolivia: 1222 especies, Hermann 1976. Colombia: 750 especies, esta publicación. Esta reseña refleja claramente las actividades del eminente briólogo alemán, el Dr. Th. Herzog, en Bolivia en la primera parte de este siglo. En los demás países, especialmente en el Perú, la información disponible es bastante escasa.

En la composición y el orden de las familias se sigue a Brotherus (1924-25) salvo en los casos señalados. Por ejemplo, Amphidium se considera nuevamente como perteniciente a las Orthotrichaceae según estudios recientes (Lewinsky, 1976). Acerca del rango de los géneros incluidos en los Hookeriales se han publicado nuevas ideas (Miller, 1971; Crosby, 1974). La familia de las Pilotrichaceae es uno de los grupos en discusión y ahora el nombre no es legítimo, ya que el nombre genérico Pilotrichum ha sido cambiado en Callicosta (Crosby, 1978). Sin embargo seguimos aquí la clasificación de Brotherus, porque todavia falta la publicación de un nuevo concepto final. No hemos usado los nombres genéricos de Calyptrochaeta (correcto para Eriopus), y Schizomitrium (correcto para Callicostella), porque todavia no se han publicado las nuevas combinaciones con los epítetos específicos de estos musgos colombianos. Según los autores una lista de recopilación basado en datos bibliográficos, no debe contener nuevas combinaciones hechas sin ver el material original.

Fué necasario modificar la nomenclatura en cerca de 600 datos obtenidos de la

${ }^{1}$ I thank A. M. Cleef for his encouragement and assistance in completing this publication and Dr. S. R. Gradstein, Dr. W. D. Margadant and Dr. F. A. Stafleu for advice in taxonomical problems. I am also grateful to Dr. E. Forero and Mrs. A. van der Hammen-Malo for kindly correcting the Spanish translation.

${ }^{2}$ Institute for Systematic Botany, Heidelberglaan 2, Utrecht, Netherlands.

$0007-2745 / 79 / 215-259 \$ 4.75 / 0$ 
literatura; las referencias originales se encuentran en la lista de sinónimos que aparece al final del trabajo. La nomenclatura se basa principalmente en el Index Muscorum (1959-1969). Los cambios propuestos en estudios mas recientes se incluyen también en la lista de sinónimos.

La literatura más antigua contiene muchas descripciones de especies nuevas. Esos datos se indican en la presente lista como "n.sp." Las nuevas especies de la primera enumeración de Hampe (1862) fueron descritas en otra parte (Linnaea 31 \& 32; 1862 , 1863); no obstante, en este caso también se emplea la denominación "n.sp."

Las colecciones indicadas con* fueron hechas por T. van der Hammen y R. Jaramillo Mejía, por A. M. Cleef, y por mi esposo, durante sus investigaciones fitosociológicas en las selvas andinas y páramos colombianos. Esos ejemplares, que alcanzan a unos 4000 números, están depositados en el Herbario Nacional Colombiano (COL), del Instituto de Ciencias Naturales, Bogotá, y en el Herbario del Instituut voor Systematische Plantkunde, Utrecht (U). De cado departamento no citado antes, se refiere únicamente una collección. Los siguientes briólogos colaboraron en el trabajo de determinación del material: H. Ando, I. Bruggeman-Nannenga, J.-P. Frahm, D. Griffin, A. Koponen, H. Ochi, y R. H. Zander. Aunque todavía continua el estudio de dichas colecciones, consideré util publicar ahora los datos obtenidos de estos especialistas, a quienes expreso mis agradecimientos. Además reconozco los aportes del Dr. K. Mägdefrau con datos del material colectado por el en Colombia (también indicados con*), y identificado por el Dr. H. Crum. La lista presentada aquí contiene todos los taxa de musgos conocido por nosotros hasta Enero de 1978.

El total de 750 especies de ninguna manera dá una reseña completa de la flora de musgos de Colombia. Todavía falta muchísimo trabajo: hacer inventarios, coleccionar material en prácticamente todo el país, y estudiarlo. En bastantes regiones del país se han coleccionado poco musgos; tal es el caso de la zona de la selva amazónica donde muy pocas especies son conocidas (leg. Schultes y Cabrera). Humboldt y Bonpland (véase Stearn, 1968), y Spruce (1908) coleccionaron a lo largo de las cabeceras del Rio Orinoco y del Río Negro, que actualmente delimitan la República de Colombia. Teniendo en cuenta que no existe la certeza de que sus colecciones provengan realmente del territorio nacional colombiano, éstas no aparecen registradas en la presente lista.

En la lista de colectores (p. 218) se encuentran datos de los colectores de musgos colombianos en orden cronológico, con las correspondientes referencias bibliográficas. Colecciones de Moritz y de Wagner, publicadas por Hampe (1847) y Müller (1847, 1852) bajo "Columbische Laubmoose," provienen de zonas que pertenecen en la actualidad a Venezuela o al Brasil, como consecuencia de los cambios fronterizos ocurridos en el siglo pasado. Por esa razón, erróneamente se han referido algunas localidades a Colombia en publicaciones recientes (Florschütz, 1964); así el material tipo: Leucobryum crispum Müll. (Moritz 131, Cocollar: Venezuela), Leucobryum giganteum Müll. (Moritz 134a, Mérida: Venezuela), Octoblepharum cocuiense Mitt. (Spruce s.n., Monte Cocui: Brasil), Octoblepharum stramineum Mitt. (Spruce s.n., San Carlos: Venezuela), Macromitrium pellucidum (Spruce 80, Río Vaupés: Brasil). El único dato para Colombia de Pilotrichum andersonii Crosby (Crosby, 1969) se debe adscribir también a Venezuela según el número de colección (90) de Wagner (fide Müller, 1852). Material coleccionado cerca del Mt. Napo (leg. Osculati) y descrito para Colombia por De Notaris como Macromitrium osculatianum, Orthodontium osculatianum, Philonotis osculatianum y Osculatia columbica proviene de una región actualmente ecuatoriana desde el Tratado de 1916. 


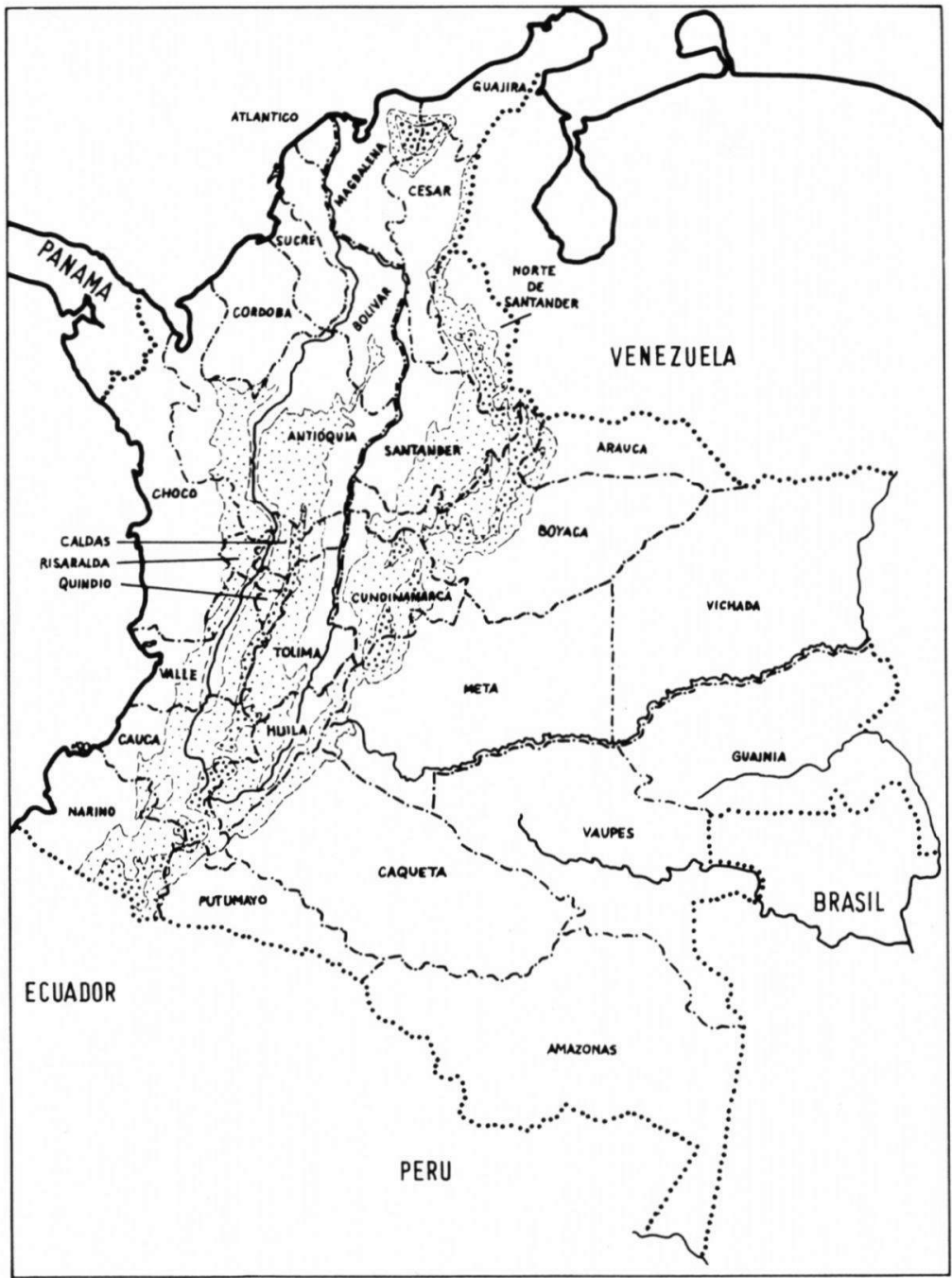

Figure 1. División territorial de Colombia.

Fué dificil localizar geográficamente muchas colecciones antiguas con indicaciones incompletas o de localidades que no pertenecen más a los departamentos actuales (Fig. 1) que han cambiado de nombre. Este problema se solucionó en gran parte con la consulta del Diccionario Geográfico de Colombia (ed. 1971) y de las cópias de mapas antiguos en el Atlas de Colombia (ed. 1969), ambos editados por el Instituto Geográfico “Angustin Codazzi” en Bogotá. Así, por ejemplo, la Provincia de Ocaña (Schlim) y la Provincia de San Pedro (Funck y Schlim) estaban localizadas en los departamentos actuales de Norte de Santander y Magdalena respectivamente. Las indicaciones de localidades de Purdie son bastante limitadas ("Páramo de Ruiz," "S. Marta," etc.). Según Ewan (1948), Purdie pasó dos veces por el flanco Sur del Macizo de la Sierra Nevada de Santa Marta. De manera que sus colecciones de "S. Marta" 
(con indicación de altura sobre el nivél del mar) podrián provenir de cualquiera de los actuales departamentos de Guajíra, Cesar o Magdalena. En este caso, cuando no se conoce la localidad por caracer de otros detalles, se usa en esta lista la denominación "Sierra Nevada de Santa Marta." El "Páramo de Ruiz" se indica como "Caldas/Tolima," porque esta región está situada en el límite de ambos departamentos. Así también el "Páramo de Quindío" (Humboldt y Bonpland) está localizado en los departamentos actuales de Quindio y Tolima; para mayor seguridad se utiliza en la lista la denominación "Quindío/Tolima." Estos mismos botánicos recogieron musgos "inter Pasto et Almaguer," según las etiquetas. Dichas colecciones las hemos registrado como "Cauca/Nariño."

La localidad "inter Bucaramanga et Pamplona" de las etiquetas de la colección de Weir, es interpretada como "Santander/Norte de Santander." Este colector inglés trabajó también a lo largo del Río Magdalena sin registrar la localidad exacta, indicando únicamente la altura sobre el nivél del mar. Dichas colecciones figuran aqui como "Fl. Magdalena."

Hampe (1865-66), cuando se refiere a las colecciones de Lindig, siempre cita 2 localidades en la Cordillera Oriental colombiana ("Andes Bogotenses"): Bogotá y el sitio propiamente dicho. En esta lista presentamos el Departamento actual, donde está situada esta segunda localidad. Por ejemplo Trematodon brevirostris Hampe es citado por Hampe (l.c.): "Bogotá, Pie de Cuesta, 1200 met., . . . . . ." y referido por nosotros como "Santander." "Río Negro, altit. $1200 \mathrm{~m}$ " de Lindig, dada la altura, estaba localizado en el Tolima; "Río Negro $2000 \mathrm{~m}$ " de Wallis se ha podido localizar en Antioquia, porque gran parte de sus colecciones provenian de ese departamento y aparentemente Wallis no coleccionó en el Tolima (véase también Barkley, 1949). La localidad "Canoas," mencionada con frecuencia por Lindig, constituye otro problema. Ewan (1948) identificó “Canoas," registrado por Purdie, como el pueblo actual de San Carlos en las cabeceras del Rio Nare en Antioquia. Pero esta localidad está fuera del área de colecciones de Lindig. En el mapa de la Sabana de Bogotá y alrededores de la CAR (Ortiz, 1963), hemos encontrado el nombre "Canoas" indicando una finca en las orillas del Río Bogotá unos $9 \mathrm{~km}$ al Sur de Mosquera. Esta sitio nos pareció como la localidad mas probable de las colecciones de Lindig y está situado en el actual Departamento de Cundinamarca. "Monte del Morro" y "Tocarema," por donde Lindig también coleccionó, no se han podido localizar; pero según el orden del itinerario es muy probable, que se trate de localidades cundinamarquesas. Finalmente, no ha sido posible establecer la localización de "Osson," citado por Mitten de una colección de Purdie.

Hace algunos años se creo el Departamento de Casanare en la parte llanera del antiguo Departamento de Boyacá. En esta lista utilizamos los límites del antiguo Departamento de Boyacá según el Atlas de Colombia (ed. 1969), que actualmente forman dos departamentos: Boyacá y Casanare.

\section{Lista de Colectores}

F. H. A. von Humboldt \& A. J. A. Bonpland (1801)_Cauca, Cundinamarca, Nariño, Quindio, Tolima, Vichada. (Hooker, 1818-20; Kunth, 1822; Hampe, 1865, 1866; Mitten, 1869).

W. Purdie (1844/46)—Boyacá, Caldas, Cesar, Cundinamarca, Guajíra, Magdalena, Norte de Santander, Santander, Tolima. (Wilson, 1847; Mitten, 1869).

H. Funck (1847)-Magdalena, Norte de Santander. (Müller, 1848, 1857; Hampe, 1865, 1866; Mitten, 1869).

L. Schlim (1847/52)—Guajíra, Magdalena, Norte de Santander. (Müller, 1848, 1857; Hampe, 1865, 1866; Mitten, 1869). 
J. Triana (1853/57)-Boyacá, Cundinamarca, Nariño, Norte de Santander, Quindio. (Müller, 1857; Hampe, 1865, 1866; Mitten, 1869).

J. Goudot (1841/45)-Cundinamarca, Tolima. (Hampe, 1865, 1866; Mitten, 1869).

A. Lindig (1859/63)-Boyacá, Cundinamarca, Santander, Tolima. (Hampe, 1862, 1865, 1866; Mitten, 1869).

J. Weir (1863/64)-Boyacá, Cundinamarca, Magdalena, Norte de Santander, Santander, Tolima. (Mitten, 1869).

G. Wallis (1872/74)-Antioquia, Norte de Santander. (Müller, 1874, 1875).

F. C. Lehmann (1880/84)-Antioquia, Cauca, Cundinamarca, Nariño, Tolima, Valle. (Bescherelle, 1894).

C. F. Baker (1895/98)-Magdalena. (Kindberg, 1901).

Fr. Apollinaire-Marie (1904/06)-Boyacá, Cundinamarca. (Paris, 1906; Thériot, 1906, 1928).

H. Pittier (1905/06)—Cauca, Huila, Magdalena, Valle. (Williams, 1908; Robinson, 1967).

E. Mayor (1910/11)-Antioquia, Caldas, Cundinamarca, Tolima. (Irmscher, 1914).

E. P. Killip (1922)-Cauca, Valle. (Williams, 1925).

E. P. Killip \& A. C. Smith (1926/27)—Norte de Santander, Santander. (Williams, 1930; Robinson, 1967).

C. Troll (1928/29)—Cundinamarca. (Thériot, 1939; Herzog, 1934, 1949).

E. Aubert de la Rue (1933)-Valle. (Thériot, 1937; Reese, 1977).

W. R. Taylor (1934)-Chocó, Nariño. (Steere, 1936).

J. Cuatrecasas (1938/66)-Boyacá, Caldas, Caquetá, Cauca, Chocó, Cundinamarca, Huila, Magdalena, Meta, Nariño, Norte de Santander, Putumayo, Santander, Valle, Vaupés. (Robinson, 1967).

A. H. G. Alston (1939)—Cundinamarca, Norte de Santander, Santander. (Bartram, 1953).

R. M. King (\& R. Jaramillo-Mejía \& A. Guevara) (1965)-Caldas, Cundinamarca, Putumayo, Tolima. (Robinson, 1967).

*K. Mägdefrau (1967)-Antioquia, Boyacá, Chocó, Cundinamarca, Magdalena.

*T. van der Hammen \& R. Jaramillo-Mejía (1967 y 1974/75)-Boyacá, Cundinamarca, Santander, Tolima.

*A. M. Cleef (1971/73-Arauca, Boyacá, Caldas, Cauca, Cundinamarca, Huila, Meta, Santander, Tolima, Valle.

*P. A. Florschütz (1972 y 1975)—Arauca, Boyacá, Caldas, Cundinamarca, Tolima.

De los siguientes colectores se ha registrado ocasionalmente algunos números: Ariste-Joseph, Barclay \& Juajibioy, Blagborne (?), Correll, Charetier, Cuervo, Daniel, Dugand, de Dryander, García \& Gutiérrez, de Garganta, Grubb \& Guymer, Guzmán, Hermann, Holdridge, Holton, Martin, Mutis, Pennell, Pérez-Arbeláez, Schultes \& Cabrera, Taylor y Uribe - Uribe.

\section{Sphagnaceae}

Sphagnum capillifolium (Ehrh.) Hedw. TOLImA: Kunth, 1822 (como S. acutifolium). cUndNAMARCA: Mitten, 1869 (como S. acutifolium); Robinson, 1967 (como S. capillaceum). NORTE DE SANTANDER, VALLE: Robinson, 1967 (como S. capillaceum).

S. compactum Cand. in Lam. \& Cand. CundinamarCA: Robinson, 1967.

S. cuspidatum Ehrh. ex Hoffm. CAUCA: Bescherelle, 1894; Robinson, 1967. cundinamarCa: Irmscher, 1914 (como S. lehmannii var. equiporosum, n.var.); Robinson, 1967. CALDAS, NARIÑo, SANTANDER, VALLE: Robinson, 1967.

*S. cyclophyllum Sull. \& Lesq. ArauCa: Cleef 10341. BoyaCÁ: Cleef 1900. Cundinamarca: Cleef 1722. META: Cleef 1052. (det. Griffin).

S. erythrocalyx Hampe in C.Müll. vaUPÉs: Robinson, 1967.

S. magellanicum Brid. antioquia: Müller, 1874 (como S. wallisii, n.sp.). CundinamarCa: Hampe, 1866 (como S. andinum, n.sp.); Mitten, 1869 (como S. andinum); Irmscher, 1914 (como S. medium); Robinson, 1967. NARIÑo; Bescherelle, 1894 (como S. medium). CaUCA: Williams, 1908 (como S. medium var. purpurascens); Robinson, 1967. CALDAS, HUILA, NORTE DE SANTANDER, pUtumayo, valle: Robinson, 1967. *arauca: Cleef 10069. "Boyaca: Cleef 9391. *META: Cleef 7728. (det. Griffin).

- var. pallescens (Warnst.) C.Jens. ANTIOQuiA: Bescherelle, 1894 (como S. medium var. albescens).

S. meridense (Hampe) C.Müll. Cundinamanca: Hampe, 1866; Mitten, 1869; Robinson, 1967. ANTIOQUiA: Mitten, 1869 (como S. limbatum). CAUCA, NARIÑo: Bescherelle, 1894 (como S. coryphaeum). PUTUMAYO: Robinson, 1967. 
*S. oxyphyllum Wamst. BOYACA: Cleef 9936. CaUCA: Cleef 538. cUndiNamARCa: Cleef 9595. HUILA: Cleef 5067. META: Cleef 7731. (det. Griffin).

S. palustre L. CUNDINAMARCA: Mitten, 1869 (como S. cymbifolium).

S. pylaesii Brid. SANTANDER: Maass, 1966.

S. recuroum P.Beauv. CundinamarCa: Paris, 1906 (como S. pulchricoma); Thériot, 1906 (como S. pulchricoma); Robinson, 1967. SANTANDER: Bartram, 1953.

-var. amblyphyllum (Russ.) Warnst. CAUCA: Bescherelle, 1894. *BOYACÁ: Cleef 9230 (det. Griffin).

*S. sancto-josephense Crum \& Crosby. BOYACA: Cleef 9408. CAUCA: Cleef 535. Cundinamarca: Cleef 280. META: Cleef 7578 (det. Griffin).

S. sparsum Hampe. CUndinamarCA: Paris, 1906 (como S. apollinairei, n.sp.); Robinson, 1967. CAUCA, VALLE: Robinson, 1967.

S. subsecundum Nees in Sturm. valle: Robinson, 1967.

\section{Andreaeaceae}

Andreaea nitida Hook.f. \& Wils. CALDAS/TOLIMA: Wilson, 1847 (como A. subenervis, n. sp.); Hampe, 1866 \& Mitten, 1869 (como A. subenervis); Schultze-Motel, 1970. *BOYACÁ: Florschütz $4070 a$ (det. Florschütz).

A. rupestris Hedw. cundinamarca: Mitten, 1869 (como A. brevipes). "arauca: Cleef 8974. *BoyacA: Cleef 2152. "CALDaS: Cleef 2530. *META: Cleef 1495 (det. Griffin). "Magdalena: Mägdefrau 1205 (det. Crum).

A. subulata Harv. in Hook. CUndinamarCa: Herzog, 1934 (como A. aristata, n.sp.). BoyacA: Schultze-Motel, 1970.

\section{Fissidentaceae}

Fissidens asplenioides Hedw. CundinamarCa: Mitten, 1869; Herzog, 1934; Robinson, 1967. TOLIMA: Robinson, 1967. *BOYACÁ: van der Hammen 2795 (det. Florschütz). *MAGDALENA: Mägdefrau 1049 (det. Crum).

F. camptodontius Kindb. Magdalena: Kindberg, 1901, n.sp.

$F$. divisus Hampe. CuNDINAMARCA: Hampe, 1866, n.sp.

*F. elegans Brid. Magdalena: Mägdefrau 1097 (det. Crum).

F. flavinervis Mitt. TOLIMA/CUNDINAMARCA: Mitten, 1869, n.sp.

F. hancockianus Steere. CHOcó: Steere, 1936, n.sp.

F. intromarginatus (Hampe) Mitt. CUNDINAMARCA: Hampe, 1862, 1866 (como Conomitrium intromarginatum, n.sp.); Mitten, 1869.

F. lindigii (Hampe) Mitt. CundinamarCa: Hampe, 1862, 1866 (como Conomitrium lindigii, n.sp.); Mitten, 1869 (como F. lindigii y como F. tequendamensis, n.sp.).

*F. polypodioides Hedw. BOYACA: van der Hammen 2793 (det. Bruggeman-Nannenga). MAGDALENA: Mägdefrau 1259 'det. Crum).

F. prionodes Mont. (fo. flexinervis (Mitt.) Florsch.) FL. Magdalena: Mitten, 1869 (como $F$. flexinervis, n.sp.).

F. repandus Wils. CUNDINAMARCA: Herzog, 1934 (como F. tortilis); Robinson, 1967. *Boyacá: van der Hammen 2794.) *TOLIMA: Cleef 3268a (det. Bruggeman-Nannenga). *MAGDALENA: Mägdefrau 1094 (det. Crum).

F. rigidulus Hook.f. \& Wils. CUNDinamarCa: Mitten, 1869. *META: Cleef 7562. *SANTANDER: Hermann 25043a (det. Bruggeman-Nannenga).

F. wallisii C.Müll. ANTIOQuiA: Müller, 1874, n.sp.

\section{Ditrichaceae}

Ceratodon novo-granatensis Hampe. CUndinamarCa, GUajIRA: Hampe, 1865a, n.sp.

C. purpureus (Hedw.) Brid. CundinamarCa: Mitten, 1869; Paris, 1906. Caldas/Tolima: Irmscher, 1914. *MAGDALENA: Mägdefrau 1209 (det. Crum).

C. stenocatpus B.S.G. CUndinamarCa: Hampe, 1862; Herzog, 1934; Robinson, 1967. BoyacÁ, VALLE: Robinson, 1967.

C. venezuelensis C.Müll. CUndINAMARCA: Irmscher, 1914.

Cheilothela boliviana (Williams) Thér. CundinamarCA: Robinson, 1967.

*Distichium capillaceum (Hedw.) B.S.G. CAUCA: Cleef 557 (det. Frahm). META: Cleef 1427 (det. Florschütz). 
Ditrichum bogotense (Hampe) Broth. CundinamarCa: Hampe, 1862, 1865a (como Cynodontium bogotense, n.sp.).

D. crinale (Tayl.) O.Kuntze. CundinamaRCA: Bartram, 1953; Robinson, 1967. BOYACÁ, TOLIMA: Robinson, 1967.

*D. gracile (Mitt.) O.Kuntze. BoYACÁ: Cleef 8640. CALDAS: Florschütz 4272a. CUNDINAMARCA: Cleef 379. Tolima: van der Hammen 3254. (det. Florschütz).

D. heteromallum (Hedw.) Britt. CundinamarCA: Robinson, 1967.

D. rufescens (Hampe) Hampe. CUNDINAMARCA: Hampe, 1862, 1865a (como Leptotrichum rufescens, n.sp.); Hampe, 1869; Mitten, 1869 (como Cynontodium rufescens, también como Cynontodium strictum); Herzog, 1934; Robinson, 1967. SANTANDER: Anderson \& Bryan, 1958.

D. submersum Card. \& Herz. valle: Robinson, 1967. *ARAUCA: Cleef 9093. *BOYACA: Cleef 8740. *CundinamarCa: Cleef 3265. (det. Frahm).

Tristichium mirabile (C.Müll.) Herz. CALDAS: Robinson, 1967.

\section{Seligeriaceae}

*Blindia acuta (Hedw.) B.S.G. META: Cleef 1378 (det. Florschütz).

${ }^{*}$ B. magellanica Schimp. var. inundata (Card.) Herz. META: Cleef 7767 (det. Florschütz).

B. sonsoniae C.Müll. ANTIOQULA: Müller, 1874, n.sp.

Brachydontium flexisetum (Hampe) Par. CUNDINAMARCA: Hampe, 1862, 1865a (como Brachyodus flexisetus, n.sp.); Mitten, 1869 (como Brachyodus).

\section{Dicranaceae}

Amphidium. Véase Orthotrichaceae (Lewinsky, 1976).

Anisothecium convolutum (Hampe) Mitt. CundinamarCA: Hampe, 1866 (como Aongstroemia convoluta, n.sp.); Mitten, 1869.

A. hookeri (C.Müll.) Broth. ANDES BogotenSES: Mitten, 1869 (como A. jamesonii, hom. illeg.).

A. vaginatum (Hook.) Mitt. CAUCA/NARuNo: Hooker, 1820 y Kunth, 1822 (como Dicranum); Hampe, 1865a (como Aongstroemia); Mitten, 1869. CUndinamarCA: Hampe, 1865a (como Aongstroemia acerosa, n.sp.); Mitten, 1869. Tolima: Robinson, 1967 (como Dicranella).

Aongstroemia julacea (Hook.) Mitt. CundinamarcA: Hampe, 1862, 1865a (como Illecebraria); Mitten, 1869.

A. maculata C.Müll. NORTE de SANTANDER: Müller, 1875, n. sp.

Atractylocarpus longisetus (Hook.) Bartr. CUNDINAMARCA: Hooker, 1820 y Kunth, 1822 (como Dicranum); Hampe, 1865a (como Dicranum); Mitten, 1869 (como Dicranum); Herzog, 1934 (como Dicranodontium, también como Dicranodontium pusillum); Thériot, 1939 (como Dicranodontium. *BOYACA: Cleef 9855 . META: Cleef 7794 (det. Frahm.). *SANTANDER: van der Hammen 1651. *Tolima: van der Hammen 3343. (det Florschütz).

A. spiripes (C.Müll.) Herm. ANTIOQULA: Müller, 1874 (como Dicranum spiripes, n.sp.).

Bruchia lindigiana (Hampe) Broth. Cundinamarca: Hampe, 1865a (como Sporledera lindigiana, n.sp.); Mitten, 1869 (como Sporledera).

B. subenervis (Hampe) Broth. CundinamaRCA: Hampe, 1865a (como Sporledera subenervis, n.sp.); Mitten, 1869 (como Sporledera).

Campylopodium curvisetum (Hampe) Par. CundinamarCa: Hampe, 1865a (como Aongstroemia curviseta, n.sp.); Mitten, 1869 (como Dicranella).

Campylopus annotinus Mitt. CUNDINAMARCA: Mitten, 1869, n.sp.; Herzog, 1934 (fo. minor). CAUCA: Robinson, 1967. *BOYACÁ: van der Hammen 2873 (det. Florschütz).

C. apollinairei Thér. CUNDINAMARCA: Thériot, 1928. BOYACÁ: Robinson, 1967.

C. arctocarpus (Hornsch.) Mitt. BOYACÁ, CUNDINAMARCA: Robinson, 1967.

C. areodictyon (C.Müll.) Mitt. CUNDinamarCa: Mitten, 1869. CaUCa: Robinson, 1967. *aRauCa: Cleef 9026. *META: Cleef 7895. (det. Frahm).

C. argyrocaulon (C.Müll.) Broth. ANTIOQUIA: Müller, 1874 (como Dicranum argyrocaulon, n.sp.). Según nuestra opinión las colecciones citadas por Robinson (1967) como C. argyrocaulon pertenecen a $C$. pittieri. * CundinAmaRCA: Mägdefrau 1366 (det. Crum).

*C. benedictii Herz. BOYACÁ: Florschütz 4108. META: Cleef $1160 a$ (det. Florschütz).

C. bogotensis Thér. CundinamaRCa: Thériot, 1939, n.sp.

*C. breweri Bartr. BOYACÁ: Cleef 2125a. CUNDINAMARCA: van der Hammen 3101. META: Cleef 1384. TOLIMA: van der Hammen 3341. (det. Florschütz). 
*C. cavifolius Mitt. arauca: Cleef 9150. BoyaCA: Cleef 7293. CaUca: Cleef 658. cundinamarCA: Cleef 5269. META: Cleef 1361. (det. Frahm).

C. chionophilus (C.Müll.) Mitt. CundinamarCa: Hampe, 1862, 1865a (como Dicranum); Mitten, 1869; Herzog, 1934. BoYACA, SANTANDER: Hampe, 1865a (como Dicranum); Mitten, 1869. Como Müller (1849) indica en la descripción de la especie, el material tipo está mezclado con Atractylocarpus longisetus: "ubi inter Dicr. longisetum nives habitat - Funck et Schlim in Coll. Linden No. 996 intermixt." (Syn. I: 398). Este hecho causa bastante confusión. 1. Brotherus cita bajo $C$. chionophilus material perteneciente a Dicranodontium o Atractylocarpus (Florschütz, 1964). 2. Robinson (1967) identifica un fragmento del material tipo (NY) como Dicranodontium denudatum. 3. Por eso Frahm (1975) indica como neotipo el material colectado por Lindig y citado por Mitten (1869). Como se quemó el material del holotipo en Berlin, será necesario localizar a los isotipos.

C. chrismarii (C.Müll.) Mitt. CUNDINAMARCA: Herzog, 1934 (como C. friabilis). BoYACA, CAUCA: Robinson, 1967.

C. chrysodictyon (Hampe) Mitt. CUNDiNAMARCA: Hampe, 1866 (como Dicranum chrysodictyon, n.sp.); Mitten, 1869. *HuILA: Cleef 4990 (det. Frahm).

C. concolor (Hook.) Brid. NARIÑo: Hooker, 1820 y Kunth, 1822 (como Dicranum); Hampe, 1865a (como Dicranum); Mitten, 1869; Thériot, 1928. BoYACA, CUNDINAMARCA: Hampe, 1865a (como Dicranum); Mitten, 1869; Paris, 1906. NORTE DE SANTANDER/SANTANDER: Mitten, 1869. *HUILA: Williams, 1908.

C. cuatrecasii H.Robins. BOYACA, CAUCA: Robinson, 1967, n.sp.

C. cucullatifolius Herz. CUNDINAMARCA, PUTUMAYO, VALLE: Robinson, 1967. *BOYACA: Cleef 1820. *CAUCA: Cleef 2636. *HUILA: Cleef 5084. *META: Cleef 8206. (det. Florschütz).

${ }^{*}$ C. densicoma (C.Müll.) Par. CundinamarCa: Cleef 9658 (det. Frahm).

C. filicaulis (Hampe) Mitt. CUNDiNamarCa: Hampe, 1866 (como Dicranum filicaule, n.sp.); Mitten, 1869.

${ }^{*}$ C. filicuspis Broth. in Herz. BOYACA: Cleef 9896 (det. Frahm).

C. filifolius (Hornsch.) Mitt. SANTANDER: Hampe, 1866 (como Dicranum porphyreodictyon); Mitten, 1869 (como C. porphyreodictyon). ANTIOQUIA: Irmscher, 1914 (como C. porphyreodictyon). PUTUMAYO: Robinson, 1967 (como C. porphyreodictyon). *BOYACÁ: van der Hammen 2889. *TOLIMA: Echeverri 828 col. (det. Florschütz).

C. flexuosus (Hedw.) Brid. boYaCA, CaUCA, CUndinamarCa, PUTUmayo: Robinson, 1967.

C. fragilis (Brid.) B.S.G. CALDAS, CAUCA: Robinson, 1967. *ARAUCA: Cleef 8971a. *BOYACA: Cleef 5764. ${ }^{*}$ CundinamarCa: Florschütz 3900. *META: Cleef 8169. (det. Florschütz).

${ }^{*} C$. harpophyllus Herz. BOYACA: Cleef 5947 (det. Frahm).

C. heterophyllus Mitt. NORTE DE SANTANDER/SANTANDER: Mitten, 1869, n.sp. BoyaCÁ: Robinson, 1967.

${ }^{*}$ C. huallagensis Broth. HUILA: Cleef 5037 (det. Frahm).

C. incertus Thér. in Herz. CUNDINAMARCA: Herzog, 1934, n.sp.; Thériot, 1939. "BoyaCA: Cleef 2138 (det. Florschütz).

C. jamesonii (Hook.) Jaeg. CundinamarCa: Hampe, 1862, 1865a (como Dicranum); Thériot, 1928; Herzog, 1934. ANTIOQUIA: Müller, 1875 (como Dicranum altissimum, n.sp.). *BOYACÁ: Cleef 1820. *META: Cleef 806. *SANTANDER: van der Hammen 2881. (det. Florschütz).

${ }^{*}$ C. jugorum Herz. BOYACÁ: Cleef 8696 (det. Frahm).

C. kingii H.Robins. *CUNDIMAMarCA: Robinson, 1967, n.sp.

C. lamellinervis (C.Müll.) Mitt. valle: Robinson, 1967 (como C. penicillatus). *CundinamaRCA: Mägdefrau 1538 (det. Crum).

C. lamprodictyon (Hampe) Mitt. CUndinamarca: Hampe, 1866 (como Dicranum lamprodictyon, n.sp.); Mitten, 1869. BOYACÁ: Robinson, 1967.

C. leucognodes (C.Müll.) Par. CundinamarCA: Herzog, 1934. valle: Robinson, 1967. *BoyaCÁ: Florschütz 4034. *CALDAS: Florschütz 4269. (det. Florschütz).

C. mexiae Bartr. et Thér. ex Frahm, nom. nud. NARIÑo: Frahm, 1975 (como C. ynesiae, nom.herb., Ynes Mexia $7630 \mathrm{NY}$ ).

C. oblongus Thér. CUNDINAMARCA: Thériot, 1939, n.sp.

C. occultus Mitt. CundivamarCa: Herzog, 1934.

${ }^{*}$ C. paramoensis Bowers. BOYACA: Cleef 4551 (det. Frahm).

C. pauper (Hampe) Mitt. CUNDINAMARCA: Hampe, 1862, 1865a (como Dicranum pauperum, n.sp.); Hampe, 1865a (como Dicranum propinquum, n.sp.); Mitten, 1869 (como C. propinquus); Robinson, 1967. ANTIOQUIA: Robinson, 1967. 
-var. minor (Hampe) Jaeg. CUNDINAMARCA: Hampe, 1865a (como Dicranum pauperum var. minus, n.var.).

C. pilifer Brid. CaUCa, CUNDinamarCa, valle: Robinson, 1967 (como C. introflexus); Sipman identificó la colección de King (C-762) de Cundinamarca como C. pilifer $(=C$. polytrichoides). *ARaUCa: Cleef 9181. *BoyaCÁ: Cleef 4705. (det. Sipman).

C. pittieri Williams. CaUCA: Williams, 1908, n.sp. CundinamarCa: Herzog, 1934 y Thériot 1939 (como Paraleucobryum densifolium, n.sp.); Bizot, Pierrot \& Pocs, 1974 (como Bizotia densifolia). valle: Robinson, 1967 (véase bajo C. argyrocaulon). *ARAUCA: Cleef $8887 a$ (det. Frahm). *BOYACÁ: Florschütz 4038. *CALDAS: Florschütz 4360. *META: Cleef 808 (det. Florschütz).

- var. congestum (Thér.) comb. nov. (Paraleucobryum densifolium Thér. var. congestum Thér., Rev. Bryol. Lichénol. 11: 66. 1939). CundinamarCa: Herzog, 1934 y Thériot, 1939.

- var. latilimbatum (Thér.) comb. nov. (Paraleucobryum densifolium Thér. var. latilimbatum Thér., Rev. Bryol. Lichénol. 11: 66. 1939). CundinamarCA: Herzog, 1934 y Thériot, 1939.

C. reflexisetus (C.Müll.) Broth. ANTIOQUiA: Müller, 1874 (como Dicranum reflexisetum, n.sp.).

C. revolvens Herz. in Thér. CAQUETA: Thériot, 1939, n.sp.

C. richardii Brid. CUNDINAMARCA: Hampe, 1865 a (como Thysanomitrium muelleri, n.sp.); Mitten, 1869; Herzog, 1934 (como Thysanomitrium); Robinson, 1967. ANTIOQUIA: Irmscher, 1914 (como Pilopogon muelleri). NORTE DE SANTANDER: Robinson, 1967. *BOYACÁ: Cleef 5918. *META: Cleef 1496. *SANTANDER: van der Hammen 1763. *TOLIMA: van der Hammen 3291. (det. Florschütz).

C. rosulatus (Hampe) Mitt. CUNDINAMARCa: Hampe, 1862, 1865a (como Dicranum rosulatum, n.sp.; 1862-var. iteratum, nom. nud.); Mitten, 1869; Paris, 1906; Thériot, 1906; Herzog, 1934 (fo. brevifolia); Thériot, 1939 (fo. brevifolia). *BOYACÁ van der Hammen 2861 (det. Florschütz).

${ }^{*}$ C. savannarum (C.Müll.) Mitt. BOYACÁ: van der Hammen 2849 (det. Florschütz).

C. strictulus (C.Müll.) H.Robins. ANTIOQUIA: Müller, 1874 (como Dicranum strictulum, n.sp.). CaUCa: Robinson, 1967. *ArauCa: Cleef 8923. "BOYACA: Cleef 7056a. "Cundinamarca: Cleef 1636. *META: Cleef 1408. (det. Frahm y Florschütz).

C. subconcolor (Hampe) Mitt. CUNDINAMARCA: Hampe, 1862, 1865a (como Dicranum subconcolor, n.sp.); Mitten, 1869; Paris, 1906. BOYACÁ, CAUCA, VAlle: Robinson, 1967. *ARAUCA: Florschútz 4120. *CALDAS: Florschütz 4266. *META: Cleef 1148. (det. Florschütz).

${ }^{*}$ C. surinamensis C.Müll. BOYACÁ: Florschütz 3979 (det. Florschütz).

C. tequendamensis Thér. in Herz. CundinamarCa: Herzog, 1934 y Thériot, 1939, n.sp.

${ }^{*}$ C. thysanomitrioides Thér. BOYACA: Cleef 5773. CaUCA: Cleef 579a (det. Frahm).

C. trichophorus Hampe ex Herz. CundinamarCa: Robinson, 1967. *BoyaCÁ: Cleef 9495 (det. Frahm).

C. tunariensis Herz. CundinamarCa: Herzog, 1934. *arauca: Cleef 8971. *Boyacá: Cleef 5876. *CALDAS: Cleef 2517. "CAUCA: Cleef 653. *META: Cleef 1285. *Tolima: van der Hammen 3340. (det. Florschütz).

${ }^{*} C$. yungarum Herz. CUNDiNAMARCA: van der Hammen 2847 (det. Florschütz).

Chorisodontium speciosum (Hook. f. \& Wils.) Broth. SIN/LOC.: Wilson, 1847 (como Dicranum speciosum, n.sp.); Hampe, 1865a (como Dicranum); Mitten, 1869 (como Dicranum). CaUCA: Williams, 1908 (como Dicranum). CundinamarCA: Herzog, 1934.

C. wallisii (C.Müll.) Broth. ANTIOQUiA: Müller, 1874 (como Dicranum wallisii, n.sp.). NARIÑo: Bescherelle, 1894 (como Dicranum), CundinamarCa: Irmscher, 1914 (como Dicranum).

Dicranella angustifolia Mitt. CUNDINAMARCA: Mitten, 1869, n.sp.

D. bogotensis (Hampe) Mitt. CundinamarCA: Hampe, 1865a (como Aongstroemia bogotensis, n.sp.); Mitten, 1869.

D. callosa (Hampe) Mitt. Cundinamarca: Hampe, 1862, 1865a (como Aongstroemia callosa, n.sp.); Mitten, 1869.

D. consimilis (Hampe) Mitt. cundinamarca: Hampe, 1866 (como Aongstroemia consimilis, n.sp.); Mitten, 1869.

D. ditissima (Hampe) Mitt. Cundinamarca: Hampe, 1862, 1865a (como Aongstroemia ditissima, n.sp.); Mitten, 1869 .

D. heteromalla (Hedw.) Schimp. Cundinamarca: Hampe, 1862, 1865a (como Aongstroemia crassinervis, n.sp.); Mitten, 1869.

D. hilariana (Mont.) Mitt. CAUCA, TOLIMA: Robinson, 1967. 
D. mayorii Broth. \& Irmsch. ANTIOQUIA: Irmscher, 1914, n.sp.

D. perrottetii (Mont.) Mitt. SIN/LOC.: Wilson, 1847 (como Dicranum sclerocarpum, n.sp.). SANTANDER: Mitten, 1869. VALLE: Williams, 1908; Robinson, 1967).

D. strumulosa (Hampe) Mitt. CundinamarCa: Hampe, 1862, 1865a (como Aongstroemia strumulosa, n.sp.); Mitten, 1869. *MAGDAlenA: Mägdefrau 1119 (det. Crum).

-var. minor (Hampe) Jaeg. CundinAMARCA: Hampe, 1865 (como Aongstroemia strumulosa var. minor, n. var.).

D. subinclinata Lor. CAUCA, PUTUMAYo, TOLIMA: Robinson, 1967.

Dicranodontium denudatum (Brid.) Britt. in Williams. HUILA: Robinson, 1967.

D. setosum Williams. CAUCA: Williams, 1908, n.sp.

Dicranum bolivianum C.Müll. (fo. flaccidissimum (C.Müll.) Herz.) ANTIOQUiA: Müller, 1874 (como D. flaccidissimum, n.sp.).

D. deflexicaulon C.Müll. ANTIOQuia: Müller, 1874, n.sp.

D. frigidum C. Müll. cundinamarCa: Hampe, 1862, 1865a; Mitten, 1869; Paris, 1906; Thériot, 1906; Herzog, 1934; Robinson, 1967. BOYACA: Hampe, 1865a; Robinson, 1967. CAUCA: Bescherelle, 1894; Williams, 1908. ANTIOQUIA, CALDAS, TOLIMA, VALlE: Robinson, 1967. *SANTANDER: van der Hammen 1618 (det. Florschütz).

- var. brevifolium Herz. CundiNamanCa: Herzog, 1934, n.var.

D. goudotii Hampe. TOlima: Hampe, 1865a, n.sp.; Mitten, 1869.

D. paramicola C.Müll. ANTIOQUiA: Müller, 1874, n.sp.

D. strictiusculum Hampe. CUNDINAMARCA: Hampe, 1862, nom.nud.

D. vesiculare Hampe. CundinamarCA: Hampe, 1866, n.sp.; Mitten, 1869.

Holomitrium crispulum Mart. CUNDINAMARCA: Hampe, 1862. CAUCA: Bescherelle, 1894.

H. lehmannii Besch. CAUCA: Bescherelle, 1894, n.sp.

H. longifolium Hampe. CUNDINAMARCA: Hampe, 1865a, n.sp.; Mitten, 1869.

Kingiobryum paramicola H.Robins. CUNDINAMARCA: Robinson, 1967, n.sp. ${ }^{*}$ META: Cleef 8086 (det. Florschütz).

Leucoloma macrodon (Hook.) Jaeg. CUndinamarCA: Hampe, 1862, 1865a (como Dicranum).

L. procumbens (Mitt.) Jaeg. CUNDINAMARCA: Mitten, 1869 (como Poecilophyllum procumbens, n.sp.).

L. serrulatum Brid. valle: Robinson, 1967. *Magdalena: Mägdefrau 1335 (det. Crum).

Microdus densus (Hook.) Besch. in Par. Tolima: Hooker, 1820 (como Dicranum); Mitten 1869 (como Dicranella).

M. lindigianus (Hampe) Besch. in Par. SANTANDER: Hampe, 1865a (como Seligeria lindigiana, n.sp.); Mitten, 1869 (como Dicranella).

M. macrocarpus (Broth. \& Irmsch.) Broth. ANTIOQuIA, CALDAS: Irmscher, 1914 (como Dicranella macrocarpa, n.sp.).

M. muralis (Hampe) Par. CUndinamarca: Hampe, 1865a (como Aongstroemia muralis, n.sp.); Mitten 1869 (como Dicranella).

M. pusillus (Hampe) Besch. in Par. SANTANDER: Hampe, 1865a (como Aongstroemia pusilla, n.sp.); Mitten, 1869 (como Dicranella).

Oreoweisia bogotensis (Hampe) Mitt. cundinamarCa: Hampe, 1862, 1865a (como Weissia bogotensis, n.sp.); Mitten, 1869 (como O. bogotensis, y también como O. ligularis, n.sp.); Herzog, 1934 (como O. ligularis). *CALDAS: Cleef 2533 (det. Zander).

*Orthodicranum flagellare (Hedw.) Loeske. BOYACÁ: van der Hammen 2601 (det. Florschütz).

Pilopogon gracilis (Hook.) Brid. QUINDIO/TOLIMA: Hooker, 1818 (como Didymodon gracile, n.sp.); Kunth, 1822 (como Didymodon). CundinamarCA: Hampe, 1862, 1865a; Mitten, 1869; Paris, 1906; Irmscher, 1914; Herzog, 1934; Robinson, 1967. Caldas/TOLIMA: Irmscher, 1914. NORTE DE SANTANDER: Bartram, 1953. BOYACÁ, CALDAS, CAUCA, PUTUMAYO, TOLIMA: Robinson, 1967. *MAGDALENA: Mägdefrau s.n. (det. Crum).

P. laevis (Tayl.) Thér. Cundinamarca: Hampe, 1865a (como $P$. piliferus, n.sp. var. proliferocomosus, nom.nud.); Mitten, 1869 (como P. piliferus). *BOYACA: Cleef 4236. *CAUCA: Cleef 9114. (det. Frahm).

P. longirostratus Mitt. VALLE: Robinson, 1967.

P. nanus Hampe. CundinamarCA: Hampe, 1862, 1865a, n.sp.; Mitten, 1869; Herzog, 1934.

P. paleaceus C.Müll. ANTIOQuia: Müller, 1874, n.sp.

Rhabdoweissia crenulata (Mitt.) Jameson. CUndinAmarca: Zander, 1977a.

R. fugax (Hedw.) B.S.G. CUNDINAMARCA: Hampe, 1865a (como Weissia lindigiana, n.sp.); Mitten, 1869 (como R. lindigiana).

Schliephackea meteorioides (Williams) Broth. VALLE: Robinson, 1967.

S. prostrata C.Müll. ANTIOQUIA: Müller, 1875, n.sp. 
Sphaerothecium phascoides (Hampe) Hampe. CUNDINAMARCA: Hampe, 1862 (como Thysanomitrium phascoides, n.sp.); Hampe, 1865a (como S. comosum, nom. illeg.); Mitten, 1869 (como S. comosum); Hampe, 1869.

-var. elatius (Hampe) Wijk \& Marg. Cundinamarca: Hampe, 1865a (como S. comosum var. elatius, n.var.); Mitten, 1869 (como S. comosum var. elatius).

Symblepharis lindigii Hampe. CundinamarCA: Hampe, 1862, 1865a, n.sp.; Mitten, 1869.

Trematodon brevirostris Hampe. SANTANDER: Hampe, 1866, n.sp.; Mitten, 1869.

\section{Leucobryaceae}

Leucobryum antillarum Schimp. ex Besch. VAlle: Robinson, 1967. CundiNAMARCA: van der Hammen 2598. *SANTANDER: van der Hammen 1734. (det. Florschütz).

L. giganteum C.Müll. VALLE: Robinson, 1967.

L. glaucum (Hedw.) Ångst. CUNDINAMARCA: Hampe, 1865a (como L. vulgare, nom. illeg.); Mitten, 1869.

L. martianum (Hornsch.) Hampe. SANTANDER: Hampe, 1865a; Mitten, 1869. PUTUMaYo, VALLE: Robinson, 1967.

*L. polakowskyi (Besch.) Card. Magdalena: Mägdefrau 1265 (det. Crum).

Ochrobryum obtusifolium (C.Müll.) Mitt. MAgDALENA: Müller, 1857 (como Leucobryum obtusifolium, n.sp.); Hampe, 1865a (como Schistomitrium); Mitten, 1869.

Octoblepharum albidum Hedw. CUNDINAmarCA: Hampe, 1862, 1865a. BoYaCA: Hampe, 1862, 1865a (var. violaceum, nom. nud.). TOLIMA: Hampe, 1862, 1865a; Mitten, 1869. SANTANDER: Hampe, 1865a. magdalena: Kindberg, 1901. VAlle: Williams, 1908. PUTUMAYO, VaUPÉs: Robinson, 1967.

*O. cocuiense Mitt. META: Idrobo 464 col (det. Florschütz).

*O. pellucidum C.Müll. NORTE DE SANTANDER: Bischler 2566 coL (det. Florschütz).

O. pulvinatum (Dozy \& Molk.) Mitt. FL. MAgdalena: Mitten, 1869. NARIN̄o: Steere, 1936. PUTUMAYO: Robinson, 1967.

\section{Calymperaceae}

Calymperes bartramii Reese. VAupt́s: Reese, 1961, n.sp.

C. erosum C.Müll. NARuÑo: Steere, 1936 (como C. donellii, corección por este autor); Reese, 1961.

C. lonchophyllum Schwaegr. PUTUMAyo: Robinson, 1967.

C. richardii C.Müll. NORTHERN COLOMBIA: Reese, 1961.

C. uleanum Broth. SIN/LOC.: Reese, 1961.

Syrrhopodon cryptocarpos Dozy \& Molk. PUTUMAYo: Robinson, 1967. Amazonas, VaupÉs: Reese, 1977.

S. gaudichaudii Mont. ANDES Bogotenses: Mitten, 1869 (como S. inflexus); Reese, 1978.

S. helicophyllus Mitt. AMAZONAS: Reese, 1978.

S. hornschuchii Mart. AMAzONAS, CAQUETA, VAUPÉs: Reese, 1977.

S. incompletus Schwaegr. PUTUMAYO: Robinson, 1967. ${ }^{*}$ CHOCo: Mägdefrau 1498 (det. Crum).

S. leprieurii Mont. Santander: Mitten, 1869 (como S. pusillus, n.sp.); Reese, 1978. Valle: Thériot, 1937 (como S. iridans).

S. lycopodioides (Brid.) C.Müll. GuajıRA: Müller, 1857; Hampe, 1865a; Mitten, 1869. CuNDINAMARCA: Mitten, 1869. CAUCA: Bescherelle, 1894. SIN/LOC.: Reese, 1978. *Magdalena: Mägdefrau 1068 (det. Crum).

S. parasiticus (Brid.) Besch. valle: Robinson, 1967.

S. prolifer Schwaegr. CUNDinamaRCa, SANTANDER: Hampe, 1865a; Mitten, 1869; Reese, 1978. *BOYACA: van der Hammen s.n. (det. Florschütz). *MAGDALENA: Mägdefrau 1274 (det. Crum).

S. quintasii Broth. META: Reese, 1974.

S. rigidus Hook. \& Grev. SANTANDER: Mitten, 1869. NARIÑo: Bescherelle, 1894. valle: Thériot, 1937. AMAZONAS, VAUPÉS: Reese, 1977.

S. rupestris Mitt. AMAZONAS, VAuPÉs: Reese, 1977.

S. simmondsii Steere. AMAZONAS, VAUPÉS: Reese, 1978.

\section{Pottiaceae}

Astomum lindigii (Hampe) Jaeg. CUndinamarCA: Hampe, 1865a (como Systegium lindigii, n.sp.); Mitten, 1869 (como Weissia hampei, nom. nov.). 
*Barbula appressifolia (Mitt.) Jaeg. MAGdalena: Mägdefrau 1186 (det. Crum).

B. campylocarpa (Tayl.) C.Müll. CUndinamarCa, PUTUMayo: Robinson, 1967.

B. glaucescens Hampe. Cundinamarca: Hampe, 1862, 1865a, n.sp.; Mitten, 1869 (como Tortula).

B. inaequalifolia Tayl. SANTANDER: Zander, 1968.

B. laevigata (Mitt.) Jaeg. BOYACÁ, CUNDINAMARCA: Robinson, 1967.

B. longirostris Hampe. CUNDINAMARCA: Hampe, 1865a, n.sp.; Mitten, 1869 (como Tortula).

B. novo-granatensis Hampe. CUNDINAMARCA: Hampe, 1862, 1865a, n.sp.; Mitten, 1869 (como Tortula).

-var. gracilior Hampe. CUNDINAMARCA: Hampe, 1865a, n.var.

-var. teretiuscula Schimp. in Hampe. CundiNamarca: Hampe 1865a, nom. nud.

B. rectifolia Tayl. cundinamarCA: Hampe, 1862; Mitten, 1869 (como Tortula).

B. replicata Tayl. cUNDINAMARCA: Hampe, 1862, 1865a (como Barbula apiculata, n.sp., hom. illeg.); Mitten, 1869 (como Tortula apiculata); Irmscher, 1914 (como Barbula apiculata); Robinson, 1967. BOYACA: Robinson, 1967. *MaGdaleNA: Mägdefrau 1178 (det. Crum).

B. stenocarpa Hampe. CundinamarCa: Hampe, 1865a, n.sp.; Mitten, 1869 (como Tortula).

B. subcespitosa (Hampe) Broth. CUNDINAMARCA: Hampe, 1862, 1865a (como Anacalypta subcespitosa, n.sp.); Mitten 1869 (como Weissia).

Bryoerythrophyllum arcuatum (Mitt.) Crum. CundinamaRCA: Mitten, 1869 (como Tortula arcuata, n.sp.).

B. campylocarpum (C.Müll.) Crum. CUndinamarCa: Hampe, 1862, 1865a (como Trichostomum); Hampe, 1866 (como Syrrhopodon crispatus, n.sp.); Mitten, 1869 (como Syrrhopodon crispatus); Herzog, 1934 (como Erythrophyllum); Robinson, 1967. BOYACA: Hampe, 1865a (como Trichostomum).

*B. jamesonii (Tayl.) Crum. BoyaCÁ: van der Hammen 2569. cundinamarca: Cleef 4834 (det. Zander).

Didymodon killipii Williams. SANTANDER: Williams, 1930, n.sp.

Erythrophyllopsis andina (Sull.) Zander. META: Zander, 1977a.

Gyroweisia lindigii (Hampe) Broth. Cundinamarca: Hampe, 1865a (como Hyophila lindigii, n.sp.); Hampe, 1869 (como Barbula); Mitten, 1869 (como Weissia).

Hyophila tortula (Schwaegr.) Hampe. Fl. MAgdelena: Mitten, 1869 (como Weissia).

Leptodontium brachyphyllum Broth. \& Thér. CUnDINAMARCA: Thériot, 1906, n.sp.; Robinson, 1967 [como L. gracilescens. Zander (1972) citó la misma colección]. *BOYACÁ: van der Hammen 2561 (det. Zander).

L. capituligerum C.Müll. CUndinamaRCA: Herzog, 1934 (como L. gracile); Robinson, 1967 (como L. gracile, y también como L. gracilescens); Zander, 1972. BOYACA: Robinson, 1967 (como L. gracile); Zander, 1972.

L. erythroneuron Herz. CundinamarCa: Herzog, 1934; Zander, 1972. *META: Cleef 1459 (det. Zander).

L. filicola Herz. cundinamarca, NAruÑo: Zander, 1972. *BoyacA: Cleef 3477 (det. Zander).

L. flexifolium (Dicks. ex With.) Hampe. CundinamarcA: Hampe, 1862, 1865a (como Trichostomum filescens, n.sp.); Mitten, 1869 (como L. filescens). MAGDALENA: Robinson, 1967 (como L. filescens). BOYACA: Zander, 1972.

L. fuhrmannii Broth. \& Irmsch. CUNDINAMARCA: Irmscher, 1914, n.sp.

L. longicaule Mitt. var. longicaule Zander. CUNDINAMARCA: Herzog, 1934 (como L. subgracile); Robinson, 1967. ANTIOQUIA: Robinson, 1967; Zander, 1972.

- $^{*}$ var. microruncinatum (Dus.) Zander. BOYACA: Cleef 1781. CAUCA: Cleef 592. META: Cleef 1291 (det. Zander).

L. luteum (Tayl.) Mitt. CUNDinamarca: Hampe, 1862, 1865a (como Trichostomum); Mitten, 1869; Paris, 1906; Thériot, 1906; Irmscher, 1914; Herzog, 1934; Robinson, 1967; Zander, 1972. antioquia: Zander, 1972. *BoyaCA: Cleef 1759. *CAUCA: Cleef $482 .{ }^{*}$ "SANTANDER: Cleef 3495. (det. Zander).

L. orthotrichoides (C.Müll.) Par. ANTroquia: Müller, 1874 (como Trichostomum orthotrichoides, n.sp.). Probablemente se trata del género Zygodon (véase Zander, 1972).

L. planifolium Herz. CUnDINAMARCA: Zander, 1972.

L. proliferum Herz. CALDAS/TOLIMA: Zander, 1972.

L. pungens (Mitt.) Kindb. CundiNAMARCA: Thériot, 1906 (como L. subgrimmiodes); Robinson, 1967 (como L. acutifolium); Zander, 1972. BOYACA, CALDAS/TOLIMA: Zander, 1972. "ARAUCA: Cleef 8936 (det. Zander). *Magdalena: Mägdefrau 1188 (det. Crum).

L. stoloniferum Zander. CUNDINAMARCA: Zander, 1972, n.sp. 
L. syntrichioides (C.Müll.) Kindb. ANTIOQUIA: Müller, 1874 (como Trichostomum syntrichioides, n. sp.); Zander, 1972. *Tolima: van der Hammen 3285 (det. Florschütz).

L. viticulosoides (P.-Beauv.) Wijk \& Marg.-*var. viticulosoides Zander. CAUCA: Cleef 498 (det. Zander).

- var. exasperatum (Card.) Zander. BOYACÁ: Cleef 9271 (det. Zander). -var. panamense (Lor.) Zander. CUNDINAMARCA: Hampe, 1865b (como Macromitrium undulatum, n.sp.); Mitten, 1869 (como Macromitrium ramosissimum, n.sp.); Zander, 1972. ANTIOQUIA: Müller, 1874 (como Trichostomum subcirrhifolium, n.sp.); Zander 1972. CALDAS: Robinson, 1967 (como L. sulphureum). vaLLE: Robinson, 1967 (como L. ulocalyx, y también como L. ulocalyx var. cirrhifolium). BOYACÁ, MAGDALENA, SANTANDER: Zander, 1972.

L. wallisii (C.Müll.) Kindb. ANTIOQUIA: Müller, 1874 (como Trichostomum wallisii, n.sp.). CaldaS, CundinamarCa: Robinson, 1967 (como L. vaginatum); Zander, 1972. *ARauCA: Cleef 10070. *BoyaCÁ: Cleef 2051. *CAUCA: Cleef 549. *META: Cleef 1416. (det. Zander).

Molendoa sendtneriana (B.S.G.) Limpr. META: Zander, $1977 \mathrm{~b}$.

*Morinia ehrenbergiana (C.Müll.) Thér. META: Cleef 854 (det. Zander).

*Pleurochaete luteola (Besch.) Thér. BOYACA: van der Hammen 2233 (det. Florschütz).

Pottia cucullata (Hampe) Jaeg. CUndinamarca: Hampe, 1866 (como Anacalypta cucullata, n.sp.); Mitten, 1869 (como Weissia).

Streptopogon calymperes C.Müll. ex Geh. CundinamarCA: Hampe, 1865a (como Calymperes lindigii, n.sp.); Mitten, 1869 (como Calymperes lindigii); Salmon, 1903 (como S. rigidus); Herzog, 1934 (como S. rigidus). CaUCA: Robinson, 1967.

S. cavifolius Mitt. Cundinamarca: Salmon, 1903.

S. erythrodontus (Tayl.) Wils. CundinamarCA: Hampe, 1862, 1865a; Mitten, 1869; Salmon, 1903; Herzog, 1934; Robinson, 1967. PUTUMAYO: Robinson, 1967.

S. lindigii Hampe. Cundinamarca: Hampe, 1865a, n.sp.; Mitten, 1869 (como S. lindigii, y también como S. latifolius, n.sp., y como S. setiferus, n.sp.); Salmon, 1903.

Tortula andicola Mont. BOYACÁ, CALDAS: Robinson, 1967. *ARAUCA: Cleef 9084. *META: Cleef 7974. (det. Florschütz). *MAGDalena: Mägdefrau 1190 (det. Crum).

T. bogotensis (Hampe) Mitt. CundinamarCa: Hampe, 1865a (como Barbula bogotensis, n.sp.); Mitten, 1869 (como T. bogotensis, y también como T. decidua, n.sp.).

T. fragilis Tayl. sIN/LOC.: Müller, 1857 (como Barbula trianae, n.sp.); Hampe, 1865a (como Barbula trianae); Mitten, 1869 (como $T$. trianae). CundinamarCA: Hampe, 1862, 1865a (como Barbula fragilis); Mitten, 1869; Robinson, 1967.

T. goudotii (Hampe) Mitt. TOlima: Hampe, 1865a (como Barbula goudotii, n.sp.); Mitten, 1869.

T. kingii H.Robins. CundinamaRCa: Robinson, 1967, n.sp.

T. pichinchensis Tayl. CUNDINAMARCA: Hampe, 1862, 1865a (como Barbula affinis, n.sp.): Mitten, 1869.

Trichostomopsis australasiae (Hook. \& Grev.) H.Robins. CUnDINAMARCA: Hampe, 1865a (como Barbula decolorans, n.sp.); Mitten, 1869 (como Tortula decolorans); Herzog, 1934 (como Asteriscium decolorans); Robinson, 1967 (como Barbula decolorans).

Trichostomum brachydontium Bruch. BOYACÁ: Robinson, 1967. *META: Cleef 1398 (det. Zander. "MAGDALENA: Mägdefrau 1235 (det. Crum).

T. novogranatense Broth. \& Irmsch. ANTIOQUIA: Irmscher, 1914, n.sp.

T. raapii Broth. ANTIOQUIA: Irmscher, 1914.

T. schlimii C.Müll. MAGDALENA: Müller, 1857, n.sp.; Hampe, 1865a (también como Syrrhopodon fragilis, n.sp.); Mitten, 1869 (como Tortula, y también como Syrrhopodon fragilis). CUNDINAMARCA: Hampe, 1865a (idem); Mitten, 1869 (idem).

Weissia controversa Hedw. cundiNAMARCA: Hampe, 1862 (como W. canaliculata, nom.nud.); Hampe, 1865a (como W. viridula, var. canaliculata, n.var.); Mitten, 1869.

\section{Grimmiaceae}

*Grimmia affinis Hornsch. MAGDALENA: Mägdefrau 1187 (det. Crum).

G. bogotensis (Hampe) Jaeg. CUNDINAMARCA: Hampe, 1862, 1865a (como Guembelia bogotensis, n.sp.); Thériot, 1906.

G. ovalis (Hedw.) Lindb. Cundinamarca: Mitten, 1869 (como G. ovata).

Racomitrium crispulum (Hook. f. \& Wils.) Hook. f. \& Wils. SIN/LOC.; Müller, 1857 (como Grimmia contermina); Hampe, 1865a (como R. conterminum); Mitten, 1869 (como Grimmia crispipila). CUNDINAMARCA: Herzog, 1934 (como R. crispipilum); Robinson, 1967. BOYACA: Robinson, 1967. *ARAUCA: Cleef 8850 (det. Florschütz). *MAGDalena: Mägdefrau 1061 (det. Crum). 
R. cucullatifolium Hampe. CUNDINAMARCA: Hampe, 1862, 1865a, n.sp.; Mitten, 1869 (como Grimmia); Herzog, 1934.

R. dichelymoides Herz. CUndinamarca: Herzog, 1934, n.sp.; Bartram, 1953. *Caldas: Cleef 2434 (det. Florschütz).

R. lanuginosum (Hedw.) Brid. TOLIMA: Hampe, 1865a; Mitten, 1869 (como Grimmia). NARIÑo: Robinson, 1967.

\section{Funariaceae}

Funaria bonplandii (Hook.) Broth. CundinamarCa: Kunth, 1822 (como Gymnostomum).

F. hygrometrica Hedw. CundinamarCA: Hampe, 1865a; Mitten, 1869; Robinson, 1967. CAUCA: Bescherelle, 1894. valle: Williams, 1908; Robinson, 1967. CALdAS, PUTUMAYO: Robinson, 1967. *Magdalena: Mägdefrau 1181 (det. Crum).

- var. calvescens (Schwaegr.) Mont. NARIÑo: Kunth, 1822 (como F. calvescens). CUNDINAMARCA: Hampe, 1862, 1865a (como F. calvescens); Mitten, 1869 (idem); Paris, 1906 (idem); Herzog, 1934 (idem). NORTE DE SANTANDER: Hampe, 1862, 1865a (idem). SANTANDER: Mitten, 1869 (idem). MAGDALENA: Kindberg, 1901 (idem). vallE: Williams, 1908 (idem). ANTIOQUIA: Irmscher, 1914 (idem).

F. jamesonii (Tayl.) Tayl. Cundinamarca: Hampe, 1862 (como Entosthodon); Hampe, 1865a (como Amphoritheca).

F. lindigii (Hampe) Broth. Cundinamarca: Hampe, 1865a (como Amphoritheca lindigii, n.sp.); Mitten, 1869 (como Entosthodon).

F. lutescens (Hampe) Broth. Cundinamarca: Hampe, 1865a (como Amphoritheca lutescens, n.sp.); Mitten, 1869 (como Entosthodon).

F. trumpffii (C.Müll.) Broth. CUNDINAMARCA: Hampe, 1862 (como Entosthodon pseudo-marginatus, nom. nud.); Hampe, 1865a (como Amphoritheca pseudo-marginata, n.sp.); Mitten, 1869 (como Entosthodon pseudo-marginatus).

F. undulata (Hampe) Broth. CundinamarCA: Hampe, 1865a (como Amphoritheca undulata, n.sp.); Mitten, 1869 (como Entosthodon).

\section{Splachnaceae}

Brachymitrion jamesonii Tayl. cundinamarca: Herzog, 1934 (como Tayloria); Koponen, 1977.

B. moritzianum (C.Müll.) A.Kop. CundinamarCA: Hampe, 1862, 1865a (como Tayloria); Mitten, 1869 (como Tayloria); Irmscher, 1914 (como Tayloria mayorii, n.sp.); Koponen, 1977.

Splachnobryum obtusum (Brid.) C.Müll. ATLANTICO: Robinson, 1967.

*Splachnum weberbaueri Reim. BOYACÁ: Cleef 4770. META: Cleef $9541 a$ (det. A. Koponen).

*Tayloria altorum Herz. MAgDAlENA: Mägdefrau 1565 (det. Crum).

T. scabriseta (Hook.) Mitt. CUNDINAMARCA: Hampe, 1865a (como Dissodon); Mitten, 1869. *ARAUCA: Cleef 8959. *BOYACA: Cleef 4770a. (det. A. Koponen).

*Tetraplodon mnioides (Hedw.) B.S.G. META: Cleef 1412 (det. A. Koponen).

\section{Bryaceae}

Acidodontium megalocarpum (Hook.) Ren. \& Card. Cundinamarca: Mitten, 1869 (como Bryum); Herzog, 1934.

A. rhamphostegium (Hampe) Jaeg. CundiNamarCa: Hampe, 1865b (como Cladodium rhamphostegium, n.sp.); Mitten, 1869 (como Bryum).

Anomobryum clavicaule (C.Müll.) Broth. ANTIOQUiA: Müller, 1875, (como Bryum clavicaule, n.sp.).

A. filiforme (Dicks.) Husn., hom. illeg. NARIÑo: Kunth, 1822 (como Bryum julaceum). CUNDINAMARCA: Hampe, 1862, 1865b (como Bryum prostratum); Mitten, 1869 (como Bryum prostratum, y también como Bryum julaceum); Herzog, 1934 (como A. prostratum); Robinson, 1967. VALLE: Robinson, 1967.

*A. plicatum Card. BOYACA: Cleef 4367 (det. Griffin).

A. semi-ovatum (Brid.) Besch. in Fourn. CUNDINAMARCA: Hampe, 1862, 1865b (como Bryum),

Brachymenium consimile (Mitt.) Jaeg. NORTE DE SANTANDER: Robinson, 1967.

B. daguense (Besch.) Par. valle: Bescherelle, 1894 (como Peromnium daguense, n.sp.).

B. morasicum Besch. CaUCA: Bescherelle, 1894, n.sp.

B. speciosum (Hook. f. \& Wils.) Steere. CAUCA: Bescherelle, 1894 (como Peromnium jamesonii).

Bryum argenteum Hedw. CAUCA/NARIÑO: Kunth, 1822. CUNDINAMARCA: Hampe, 1862, 1865b; Hampe, 1869 (como B. corrugatum, n.sp.); Mitten, 1869; Paris, 1906; Herzog, 1934 (fo. mi- 
crophylla); Robinson, 1967. SIN/LOC.: Williams, 1908. BOYACA, CALDAS, CAUCA, VALLE: Robinson, 1967. *MAGDALENA: Mägdefrau 1202 (det. Crum).

-var. lanatum (P. Beauv.) Hampe. CundinamarCA: Hampe, 1865b; Irmscher, 1914.

B. billardieri Schwaegr. TOLIMA: Hampe, $1865 \mathrm{~b}$ (como B. goudotii, n.sp.); Mitten, 1869 (como B. goudotii). BOYACA, CUNDINAMARCA; Robinson, 1967 (como B. truncorum).

*B. candicans Tayl. CALDAS: Cleef 2407. META: Cleef 7919. (det. Florschütz).

B. coronatum Schwaegr. SANTANDER: Hampe, 1865b. MAGDALENA: Mitten, 1869; Kindberg, 1901.

B. cruegeri Hampe in C.Müll. CAldas, PUTUmayo: Robinson, 1967 (como Pohlia).

B. dichotomum Hedw. MAGDalenA: Kindberg, 1901. ANTIOQUIA: Irmscher, 1914 (como $B$. bulbillosum).

B. erythroneuron Mitt. CUNDINAMARCA: Mitten, 1869, n.sp.

B. fragilicuspis Herz. CUNDINAMARCA: Herzog, 1934.

${ }^{*}$ B. laevigatum Hook. f. \& Wils. BOYACA: Cleef 7513 (det. Ochi).

B. leptochaeton Hampe. CUNDINAMARCA: Hampe, 1862, nom.nud.

B. lindigianum Hampe. CundiNAmarCA: Hampe, 1865b, n.sp.; Mitten, 1869; Herzog, 1934.

B. mayorii Broth. \& Irmsch. ANTIOQUIA: Irmscher, 1914, n.sp.

B. microchaeton Hampe. CUNDINAMARCA: Hampe, 1865b, n.sp.

B. procerum Schimp. ex Besch. BOYACA, CUNDINAMARCA: Robinson, 1967.

B. proliferum Mitt. CUndinamarCa: Mitten, 1869, n.sp.

B. pseudotriquetrum (Hedw.) Gaertn., Meyer \& Scherb.-subsp. bimum (Schreb.) Hartm. CUNDINAMARCA: Mitten, 1869 (como B. bimum).

B. remotifolium Hampe. CUNDINAMARCA: Hampe, 1862, 1865b, n.sp.; Mitten, 1869.

B. semi-marginatum Hampe. CundinamarCa: Hampe, 1862, 1865b, n.sp.; Mitten, 1869.

B. socorrense (Hampe) Mitt. SANTANDER: Hampe, 1865b (como Cladodium socorrense, n.sp.); Mitten, 1869. Tolima: Bescherelle, 1894.

B. sordidum Hampe. cundinamarca: Hampe, 1865b; Mitten, 1869.

B. terebellum Hampe. CundiNAMARCA: Hampe, 1862, 1865b, n.sp.; Mitten, 1869; Paris, 1906; Herzog, 1934.

Haplodontium megalocarpum (Arnott) Jaeg. CAUCA/NARIÑo: Kunth, 1822 (como Bryum turbinatum var. minus Hook., n.var.); Hampe, 1865b (como H. jamesonii); Mitten, 1869 (como Mielichhoferia megalocarpa). CUNDINAMARCA: Robinson, 1967 (como H. jamesonii).

*Leptobryum pyriforme (Hedw.) Wils. CundinAMARCA: Cleef 3062a (det. Florschütz).

Mielichhoferia antisanensis (Bartr.) H.Robins. CUNDINAMARCA: Robinson, 1967.

M. bogotensis Hampe. CundinamarCa: Hampe, 1865b, n.sp.; Mitten, 1869; Herzog, 1934; Robinson, 1967. CAUCA: Robinson, 1967.

M. brevicaulis Hornsch. CUNDINAMARCA: Mitten, 1869; Robinson, 1967. CALDAS: Mitten, 1869.

*M. campylocarpa (Hook. \& Arnott) Mitt. CUNDINAMARCA: Mägdefrau 1376. MAGDALENA: Mägdefrau 1194. (det. Crum).

M. clavellata Mitt. Cundinamarca: Mitten, 1869, n.sp.

$M$. dolichotheca Herz. Cundinamarca: Herzog, 1934, n.sp.

M. elegans Herz. cundinamarca: Herzog, 1934.

$M$. graciliseta (Hampe) Mitt. CUNDINAMARCA: Hampe, 1862, 1865b (como Leptochlaena graciliseta, n.sp.); Mitten, 1869.

M. Lindigii Hampe. CUNDINAMARCA: Hampe, 1862, 1865b, n.sp.; Mitten, 1869.

$M$. pectinata C.Müll. NORTE DE SANTANDER: Müller, 1875 , n.sp.

M. pusilla (Hook. f. \& Wils.) Mitt. CundinamarCa: Hampe, 1865b (como M. microstoma, n.sp.); Mitten, 1869; Herzog, 1934.

M. subobliqua Hampe. CUNDINAMARCA: Hanpe, 1869, n.sp.

Mniobryum wahlenbergii (Web. \& Mohr.) Jenn. CundiNAMARCA: Mitten, 1869 (como Bryum albicans).

Orthodontium longisetum Hampe. CundinamarCa: Hampe, 1862, 1865b, n.sp.; Herzog, 1934; Meijer, 1952.

O. pellucens (Hook.) B.S.G. cundinamarca: Hampe, 1865b (como O. confine, n.sp.); Mitten, 1869 (como Apalodium); Meijer, 1952. TOLIMA: Meijer, 1952.

Osculatia columbica DeNot. valle: Williams, 1925 (como Neobryum costatum, n.sp.); CAUCA: Robinson, 1967.

Pohlia flexuosa Hook. Caldas, CaQueta, CUndinamarca: Robinson, 1967.

P. fusifera (Mitt.) Broth. CAUCA, CUNDINAMARCA: Robinson, 1967.

P. integridens (C.Müll.) Broth. CundinamarCA: Hampe, 1862, 1865b y Mitten, 1869 (como 
Bryum spectabile); Herzog, 1934 (como Webera spectabilis); Robinson, 1967. BOYACA: Hampe, 1865b y Mitten, 1869 (como Bryum spectabile).

P. leptodontium (Mitt.) Broth. CundinamarCA: Mitten, 1869 (como Bryum leptodontium, n.sp.).

P. papillosa (Jaeg.) Broth. CUNDINAMARCA: Hampe, $1865 \mathrm{~b}$ (como Brachymenium, nom. nud.); Mitten, 1869 (como Bryum); Herzog, 1934 (como Webera). SANTANDER: Hampe, 1865b (como Brachymenium); Mitten, 1869 (como Bryum).

P. paucifolia (Jaeg.) Broth. SIN/Loc.: Hampe, 1865b (como Bryum paucifolium, n.sp., hom. illeg.); Mitten, 1869 (como Bryum).

P. salaminae (C.Müll.) Broth. ANTIOQUiA: Müller, 1874 (como Bryum salaminae, n.sp.).

P. subcurvata (Mitt.) Broth. CUNDINAMARCA: Mitten, 1869 (como Bryum subcurvatum, n.sp.); Robinson, 1967.

Rhodobryum beyrichianum (Hornsch.) C.Müll. in Hampe. SANTANDER: Mitten, 1869 (como Bryum). Cundinamarca: Paris, 1906. *Magdalena: Mägdefrau 1278 (det. Crum).

R. grandifolium (Tayl.) Schimp. in Par. CundinamarCa: Hampe, 1862, 1865b (como Bryum); Mitten, 1869 (como Bryum). CAUCA: Bescherelle, 1894 (como Bryum); Robinson, 1967. *ANTIOQuia: Mägdefrau 1301. *Magdalena: Mägdefrau 1331. (det. Crum).

R. mniopsis (C.Müll.) Par. ANTIOQUla: Müller, 1874 (Bryum mniopsis, n.sp.).

R. pycnopyxis (C.Müll.) Par. ANTIOQULA: Irmscher, 1914 (como Bryum).

\section{Mniaceae}

Plagiomnium rhynchophorum (Hook.) T.Kop. CUNDINAMARCA: Hampe, 1862 (como Mnium rostratum var. occidentale, n.var., nom.nud.); Hampe, 1865b y Mitten, 1869 (como Mnium rostratum); Robinson, 1967 (como Mnium rostratum var. ligulatum). CALDAS: Robinson, 1967 (idem). *Magdalena: Mägdefrau 1100 (det. Crum). Según T. Koponen (1979, en curso de publicación) Plagiomnium rhynchophorum es la única especie de Plagiomnium, sect. Rostrata en Suramérica.

\section{Phyllodrepaniaceae}

Phyllodrepanium falcifolium (Schwaegr.) Crosby. VALLE: Robinson, 1967 (como Drepanophyllum).

\section{Rhizogoniaceae}

Rhizogonium lindigii (Hampe) Mitt. Cundinamarca: Hampe, 1865b (como Mnium lindigii, n.sp.); Mitten, 1869. CAUCA: Bescherelle, 1894.

R. mnioides (Hook.) Wils. in Hook. f. MAgdalena/NoRTE DE SANTANDER: Müller, 1857 y Hampe, 1865b y Mitten, 1869 (como Mnium polycarpum). CUNDINAMARCA: Hampe, 1865b (como Mnium polycarpum).

R. spiniforme (Hedw.) Bruch. NORTE DE SANTANDER: Müller, 1848 (como Mnium). CUNDiNAMARCA: Hampe, 1862, 1865b (como Mnium); Mitten, 1869; Paris, 1906. BoYACÁ: Hampe, 1865b (como Mnium). SANTANDER: Mitten, 1869. CaUCA: Williams, 1908. Valle: Robinson, 1967. *Magdalena: Mägdefrau 1267 (det. Crum).

\section{Aulacomniaceae}

Leptotheca costaricensis Card. \& Thér. VALLE: Robinson, 1967.

\section{Meesiaceae}

Meesia lavardei Thér. valle: Thériot, 1937, n.sp.

M. longiseta Hedw. SANTANDER: Robinson, 1967.

\section{Bartramiaceae}

Anacolia laevisphaera (Tayl.) Flow. in Grout. CUNDINAMARCA: Hampe, 1862, 1865a (como Glyphocarpa taylori, nom. illeg.); Mitten, 1869 (como Bartramia subsessilis); Paris, 1906 (como Glyphocarpus); Herzog, 1934 (como A. subsessilis); Robinson, 1967. BOYACÁ: Robinson, 1967.

Bartramia angustifolia Mitt. BOYACÁ, CUNDINAMARCA, NORTE DE SANTANDER: Robinson, 1967 *CAUCA: Cleef 536. *META: Cleef 7614 (det. Griffin).

B. defoliata C.Müll. ANTIOQUIA: Müller, 1874.

B. dilatata Broth. \& Irmsch. CundinamaRCA: Irmscher, 1914, n.sp. 
B. flavicans Mitt. CundinamarCa: Hampe, 1862, 1865a (como B. bogotensis, n.sp.); Mitten, 1869. BOYACÁ: Hampe, 1865a (como B. subpatens, n.sp.); Mitten, 1869 (como B. subpatens); Robinson, 1967. CALDAS: Robinson, 1967.

B. fragilifolia C.Müll. CUNDINAMARCA: Irmscher, 1914; Herzog, 1934.

B. longifolia Hook. QUINDIO/TOLIMA: Hooker, 1818, n.sp.; Kunth, 1822; Mitten, 1869. TOLIMA: Robinson, 1967. *Magdalena: Mägdefrau 1057 (det. Crum).

*B. mathewsii Mitt. CundinamaRCA: Cleef 4033 (det. Florschütz).

B. polytrichoides C.Müll. ANTIOQUIA: Müller, 1874, n.sp.

B. potosica Mont. Tolima: Hampe, 1865a; Mitten, 1869. *ARauCA: Cleef 9006. *BoyaCÁ: Cleef 9210 (det. Griffin). *Magdalena: Mägdefrau 1179 (det. Crum).

B. strumosa (Hampe) Mitt. CUNDINAMARCA: Hampe, 1862, 1865a (como Glyphocarpa strumosa, n.sp.); Mitten, 1869; Herzog, 1934.

B. thelioides C.Müll. NORTE DE SANTANDER: Müller, 1875, n.sp.

Bartramidula lindigii (Hampe) Par. CUNDINAMARCA: Hampe, 1862, 1865a (como Glyphocarpa lindigii, n.sp.); Mitten, 1869 (como Bartramia).

Breutelia allionii Broth. BOYACA, CAUCA, PUTUMAYo: Robinson, 1967. *Caldas: Cleef 2569. *Cundinamarca: Cleef 1623. (det. Florschütz). *MAgdalena: Mägdefrau 1365 (det. Crum).

B. brittoniae Ren. \& Card. sIN/Loc.: Robinson, 1967. *CundiNAMARCA: Cleef 3842 (det. Zander).

B. chrysea (C.Müll.) Jaeg. ANTIOQUIA: Müller, 1874 (como Bartramia chrysea, n.sp.). CUNDINAMARCA: Irmscher, 1914 (como B. sphagneticola, n.sp.); Herzog, 1934. BOYACA: Robinson, 1967.

B. falcatula Broth. \& Irmsch. CALDAS/TOLIMA: Irmscher, 1914, n.sp.

B. inclinata (Hampe \& Lor.) Jaeg. CAUCA, CUNDINAMARCA, NORTE DE SANTANDER, VALLE: Robinson, 1967.

${ }^{*} B$. integrifolia (Tayl.) Jaeg. BOYACÁ: Cleef 7071. META: Cleef 8078. (det. Florschütz).

B. karsteniana (C.Müll.) Jaeg. CUNDINAMARCA: Müller, 1874 (como Bartramia karsteniana, n.sp.); CALDAS: Robinson, 1967.

B. lorentzii (C.Müll.) Kindb. CUNdinamarCA: Robinson, 1967.

B. rhythidioides Herz. CUNDINAMARCA: Herzog, 1934, n.sp.

B. squarrosa Jaeg. CUNDINAMARCA: Mitten, 1869 (como Bartramia squarrosa, n.sp.).

B. subarcuata (C.Müll.) Schimp. in Besch. CuNdiNAMARCA: Hampe, 1862, 1865a (como Bartramia); Mitten, 1869 (como Bartramia); Robinson, 1967. BOYACÁ, CALDAS, SANTANDER, VAlLE: Robinson, 1967. *CAUCA: Cleef 670 (det. Florschütz). *MAGDALENA: Mägdefrau 1324 (det. Crum).

B. tomentosa (Brid.) Jaeg. CundinamaRCA: Hampe, 1862, 1865a (como Bartramia macrotheca, n.sp.); Mitten, 1869 (como Bartramia); Paris, 1906; Robinson, 1967. CAUCA: Bescherelle, 1894. CALDAS, PUTUMAYO, TOlima: Robinson, 1967.

B. trianae (Hampe) Jaeg. CundinamarCA: Hampe, 1865a (como Bartramia trianae, n.sp.); Mitten, 1869 (como Bartramia).

Conostomum jamesonii (Tayl.) Steere. CUNDINAMARCA: Mitten, 1869 (como Bartramia humilis). Steere (1948) coloca el material de Mitten en el género Conostomum, pero según Robinson (1967) parte de esta colección claramente pertenece al género Bartramia.

*C. pentastichum (Brid.) Lindb. BOYACA: Cleef 5877. cundiNAMARCA: Cleef 5423. (det. Griffin).

Leiomela aristifolia (Jaeg.) Wijk \& Marg. CundinAMARCA: Bartram, 1953 (como L. aristata).

L. bartramioides (Hook.) Par. CundinamarCA: Hampe, 1862, 1865a (como Cryptopodium hookeri); Mitten, 1869 (como Bartramia jamesonii).

L. lopezii Griffin. CUNDINAMARCA: Griffin, 1977b.

L. lutescens (Hampe) Broth. CUNDINAMARCA: Hampe, 1866 (como Bartramia lutescens, n.sp.); Mitten, 1869 (como Bartramia).

Philonotis andina (Mitt.) Jaeg. CaUCA, Cundinamarca: Robinson, 1967.

$P$. angulata (Tayl.) Jaeg. CuNDiNAMARCA, VAlLE: Robinson, 1967 (según Robinson (1967) no idéntico con P. rufiflora, como indica el Index Muscorum). *META: Cleef 8244 (det. Griffin).

P. crassinervia Broth. \& Par. Cundinamarca: Paris, 1906, n.sp.

P. curvata (Hampe) Jaeg. CUNDINAMARCA: Hampe, 1862, 1865a (como Bartramia curvata, n.sp.); Mitten, 1869 (como Bartramia); Robinson, 1967. Valle: Robinson, 1967.

P. elegantula (Tayl.) Jaeg. CUNDINAMARCA: Hampe, 1862, 1865a (como Bartramia, var. minor, nom. nud.); Mitten, 1869 (como Bartramia); Paris, 1906; Robinson, 1967. SANTANDER: Robinson, 1967 (según Robinson (1967) no idéntico con P. rufiflora, como indica el Index Muscorum).

P. fontanella (Hampe) Jaeg. CUNDINAMARCA: Hampe, 1962, 1865a (como Bartramia fontanella, 
n.sp.); Mitten, 1869 (como Bartramia); Bescherelle, 1894; Paris, 1906; Herzog, 1934; Robinson, 1967. valle: Robinson, 1967. *CAUCA: Cleef 4982 (det. Griffin).

$P$. marchica (Hedw.) Brid. Magdalena: Kindberg, 1901 (como P. umbonata, n.sp.).

P. osculatiana DeNot. MAgdalenA: Kindberg, 1901.

$P$. revoluta Bosch \& Lac. PUTUMAYO: Robinson, 1967.

P. scabrifolia (Hook. f. \& Wils.) Braithw. Tolima: Hampe, 1865a (como Bartramia pinnata, n.sp.); Mitten, 1869 (como Bartramia pinnata). CUNDINAMARCA: Robinson, 1967.

$P$. sphaericarpa (Hedw.) Brid. CUNDinamafCA: Robinson, 1967.

P. striatula (Mitt.) Jaeg. CUNDINAMARCA: Mitten, 1869 (como Bartramia striatula, n.sp.).

$P$ uncinata (Schwaegr.) Brid. var. uncinata Florsch. CALDAS, CUNDINAMARCA, PUTUMAYo, VALLE: Robinson, 1967.

-var. glaucescens (Hornsch.) Florsch. CundinamaRCa: Hampe, 1862, 1865a (como Bartramia minuta); Hampe, 1865a (como Bartramia versifolia, n.sp.); Mitten, 1869 (como Bartramia minuta, y también como Bartramia versifolia y como Bartramia tenella). SANTANDER: Mitten, 1869 (como Bartramia tenella). ANTIOQUIA: Irmscher, 1914 (como P. tenella). NARIÑo: Steere, 1936 (como P. tenella).

-var. gracilenta (Hampe) Dism. CundinamarCa: Hampe, 1865a (como Bartramia gracilenta, n.sp.); Mitten, 1869 (como Bartramia gracilenta); Robinson, 1967. *MAGDALENA: Mägdefrau 1048 (det. Crum).

-var. gracillima (Aongstr.) Florsch. CUndinamaRCA: Robinson, 1967.

\section{Erpodiaceae}

Erpodium coronatum (Hook. f. \& Wils.) Mitt. CundiNamarCA: Crum, 1972.

Ptychomitriaceae

Ptychomitrium lindigii (Hampe) Jaeg. CundinamarCa: Hampe, 1862, 1865b (como Brachysteleum lindigii, n.sp.); Mitten, 1869 (como Glyphomitrium).

P. lobuliferum (Mitt.) Jaeg. CUNDINAMARCA: Mitten, 1869 (como Glyphomitrium lobuliferum, n.sp.).

\section{Orthotrichaceae}

Amphidium cyathicarpum (Mont.) Broth. CUNDINAMARCA: Mitten, 1869 (como Rhabdoweisia). Groutiella mucronifolia (Hook. \& Grev.) Crum \& Steere. CundinamarCA: Mitten, 1869 (como Macromitrium apiculatum). NARIÑo: Steere, 1936 (como Macromitrium mucronifolium). VALLE: Robinson, 1967 (como G. apiculata).

G. tomentosa (Hornsch.) Wijk \& Marg. Cundinamarca, PUTUmayo: Robinson, 1967 (como G. fragilis).

Macrocoma frigidum (C.Müll.) Vitt. GUajıRA: Müller, 1857 (como Macromitrium frigidum, n.sp.); Hampe, 1865b (como Macromitrium); Mitten, 1869 (como Macromitrium).

Macromitrium attenuatum Hampe. CUNDINAMARCA: Hampe, 1865b; Mitten, 1869.

$M$. aureum C.Müll. NORTE DE SANTANDER: Müller, 1857, n.sp.; Hampe, 1865b; Mitten, 1869.

$M$. cirrosum (Hedw.) Brid. valle: Bescherelle, 1894; Robinson, 1967. *arauCa: Cleef 10121 (det. Florschütz).

M. crenulatum Hampe. Cundinamanca: Hampe, 1862, 1865b, n.sp.; Mitten, 1869; Bescherelle, 1894; Paris, 1906. GuajIRA: Hampe, 1865b; Mitten, 1869. CaUCA: Bescherelle, 1894.

M. guatemalense C.Müll. sin/LOC.: Müller, 1857 (como $M$. trianae, n.sp.). CundinamaRCA: Hampe, 1862, $1865 b$ (como M. trianae); Mitten, 1869 (como M. trianae).

M. longifolium (Hook.) Brid. Cundinamarca: Hampe, 1865b; Mitten, 1869. Tolima: Hampe, 1865b. Cauca: Bescherelle, 1894. *Magdalena: Mägdefrau 1030 (det. Crum).

M. longirostre (Hook.) Schwaegr. TOLIMA: Kunth, 1822 (como Orthotrichum).

M. portoricense Williams. PUTUMAYO: Robinson, 1967 (como M. podocarpi; corección por este autor).

M. proliferum Mitt. ANDES BOGOTENSES: Mitten, 1869, n.sp.

M. punctatum (Hook. \& Grev.) Brid. Tolima: Hampe, 1865b (como M. contextum, n.sp.); Mitten, 1869 (como $M$. contextum). CUNDINAMARCA: Mitten, 1869 (como $M$. liberum, n.sp.). sin/ LOC.: Mitten, 1869.

M. richardii Schwaegr. CaUCA: Bescherelle, 1894 (como M. didymodon).

M. serrulatum Mitt. CAUCA, VALLE: Robinson, 1967. 
M. swainsonii (Hook.) Brid. CUndinamarCa, Magdalena, SANTANDER: Mitten, 1869.

M. tocatemae Hampe. CUNDinamarca: Hampe, 1862, 1865b, n.sp.; Mitten, 1869.

M. trachypodium Mitt. VALLE: Robinson, 1967.

Orthotrichum aristatum Hampe. CUNDINAMARCA: Hampe, 1862, 1865b, n.sp., hom. illeg. (var. minus, nom. nud.); Mitten, 1869.

O. elongatum Tayl. CUNDINAMARCA: Hampe, 1865b; Robinson, 1967. CAUCA: Robinson, 1967.

O. mandonii Schimp. ex Hampe. Cundinamarca: Hampe, 1865b; Mitten, 1869; Herzog, 1934; Robinson, 1967.

O. penicillatum Mitt. cundinamarca: Mitten, 1869, n.sp. * Magdalena: Mägdefrau 1227 (det. Crum).

O. pycnophyllum Schimp. in C.Müll. cundinamarca: Hampe, 1865b (como O. recurvans). *MaGdalena: Mägdefrau 1080 (det. Crum).

O. tuberculatum Mitt. CUNDINAMARCA: Mitten, 1869, n.sp.; Herzog, 1934.

O. wallisii C.Müll. ANTIOQuia: Müller, 1874, n.sp. CundinamarCA: Herzog, 1934

*Zygodon campylophyllus C.Müll. BOYACÁ: Cleef 4634. META: Cleef 7610 (det. Zander).

Z. goudotii Hampe. TOLIMA: Hampe, 1865b, n.sp.; Mitten, 1869; Malta, 1926. NORTE DE SANTANDER: Müller, 1875 (como Z. aureus n. sp.), Malta, 1926. BOYACÁ: Robinson, 1967.

Z. nivalis Hampe. TOLIMA: Hampe, 1865b, n.sp.; Mitten, 1869; Malta, 1926.

Z. obtusifolius Hook. Cundinamarca: Hampe, 1865b (como Z. linguiformis); Mitten, 1869 (como Z. linguiformis); Malta, 1926.

Z. pichinchensis (Tayl.) Mitt. SANTANDER: Williams, 1930 (como Leptodontium spinosum n.sp.); Robinson, 1967 (como Leptodontium spinosum). *ARAUCA: Cleef 8845. *BOYACÁ: Cleef 5565. (det. Zander).

Z. reinwardtii (Hornsch.) Braun in B.S.G. CUNDINAMARCA: Hampe, 1862 (como Z. moritzianus, y también como Z. denticulatus, y var. minor, nom. nud.); Malta, 1926; Robinson, 1967. CAUCA: Robinson, 1967. *BOYACA: van der Hammen 2023 (det. Florschütz). "MAGdalena: Mägdefrau 1044 (det. Crum). Z. subdenticulatus Hampe no es tratado como sinónimo de Z. reinwardtii var. subintegrifolius Malta (Véase Florschütz, 1964).

Z. rufescens (Hampe) Broth. in Par. Cundinamarca: Hampe, 1865b (como Orthotrichum rufescens, n.sp.); Mitten, 1869 (como Orthotrichum); Malta, 1926.

Z. setulosus Mitt. ANDES NOvo-Granatenses: Mitten, 1869, n.sp. CUndinamarCa: Paris, 1906; Malta, 1926.

Z. sordidus C.Müll. SIN/LOC.: Müller, 1857, n.sp. CundiNAMARCA: Hampe, 1865b; Mitten, 1869; Malta, 1926.

*Z. squarrosus (Tayl.) C.Müll. META: Cleef 8151 (det. Zander).

Z. subdenticulatus Hampe. CundinamarCA: Hampe, 1865b, n.sp.; Hampe, 1866 (como Z. stenocarpus, identificado por Malta (1926) como Z. reinwardtii var. subintegrifolius); Mitten, 1869; Malta, 1926 y Herzog, 1934 (como Z. reinwardtii var. subintegrifolius). BOYACA: Hampe, 1865b. NORTE DE SANTANDER: Müller, 1875 (como Z. ceratodontoides, n.sp.); Malta, 1926 (como Z. reinwardtii var. subintegrifolius, fo. ceratodontoides). ANTIOQULA: Irmscher, 1914.

\section{Helicophyllaceae}

Helicophyllum torquatum (Hook.) Brid. TOLima: Hampe, 1866.

Racopilaceae

Racopilum intermedium Hampe. CUNDINAMARCA: Hampe, 1862, 1866, n.sp.; Mitten, 1869.

R. tomentosum (Hedw.) Brid. cundinamarca: Hampe, 1862, 1866; Mitten, 1869; Robinson, 1967. SANTANDER: Hampe, 1866. MAGDALENA: Kindberg, 1901. ANTIOQUIA: Irmscher, 1914. TOLIMA, NORTE DE SANTANDER: Robinson, 1967. *MAGDALENA: Mägdefrau 1119 (det. Crum).

\section{Fontinalaceae}

Fontinalis bogotensis Hampe. CUndinamarCA: Hampe, 1862, 1865b, n.sp.; Mitten, 1869; Paris, 1906; Thériot, 1906; Welch, 1960; Robinson, 1967. NORTE DE SANTANDER: Bartram, 1953.

\section{Hedwigiaceae}

Hedwigia ciliata (Hedw.) P.-Beauv. CUNDINAMARCA: Hampe, 1862 (como H. subrevoluta, var. incana, nom. nud.); Mitten, 1869; Paris, 1906. ${ }^{*}$ Magdalena: Mägdefrau 1176 (det. Crum). - var. nivalis. CUNDINAMARCA: Hampe. 1865b, n.var. 
Hedwigidium integrifolium (P.-Beauv.) Dix. in C.Jens. CundinamarCA: Hampe, 1862 (como Harrisonia rhabdocarpa, n.sp.); Mitten, 1869 (como Hedwigia imberbis). *MAGDALENA: Mägdefrau 1060 (det. Crum).

Rhacocarpus purpurascens (Brid.) Par. QUINDIO/TOLIMA: Hooker, 1820 y Kunth, 1822 (como Hedwigia humboldtii); Hampe, $1865 \mathrm{~b}$ (como Harrisonia humboldtii); Mitten, 1869 (como Hedwigia humboldtii). CUNDINAMARCA: Hampe, 1862, 1865b (como Harrisonia humboldtii); Irmscher, 1914 (como R. humboldtii); Herzog, 1934 (como R. humboldtii var. orbiculatus); Robinson, 1967 (como R. humboldtii). BOYACA, CALDAS, CAUCA, Valle: Robinson, 1967 (como R. humboldtii). *ARAUCÁ: Cleef 9133. *META: Cleef 1446. *TOLIMA: van der Hammen 3222. (det. Cleef).

\section{Cryphaeaceae}

Acrocryphaea gardneri (Mitt.) Jaeg. CundinAmARCA: Mitten, 1869 (como Cryphaea).

Cryphaea lindigiana Schimp. in Par. Cundinamarca: Hampe, 1865b (como C. cuspidata Hampe, n.sp., hom. illeg.).

C. novo-granatensis Hampe. CUNDINAMARCA: Hampe, 1865b, n.sp.

C. patens Hornsch. ex C.Müll. cundinamarCa: Hampe, 1865b. "Magdalena: Mägdefrau 1174 (det. Crum).

C. pilifera Mitt. CundinamarCa: Mitten, 1869, n.sp.

\section{Leucodontaceae}

Leucodontopsis geniculata (Mitt.) Crum \& Steere. PUTUmayo: Robinson, 1967.

\section{Lepyrodontaceae}

Lepyrodon tomentosus (Hook.) Mitt. CUndinamarCA, TOLima: Hampe, 1862, 1865b (como L. suborthostichus); Mitten, 1869. CALDAS/TOLIMA: Mitten, 1869; Irmscher, 1914. CALDAS, valle: Robinson, 1967. *arauca: Cleef 9031. " BoyacA: Cleef 1791. *CaUCa: Cleef 515. *META: Cleef 883. (det. Griffin).

\section{Prionodontaceae}

Prionodon densus Hedw. (C.Müll.) SIN/Loc.: Wilson, 1847 (como Neckera). Magdalena: Müller, 1857 (como Pilotrichum lycopodium, n.sp.). cundinamaRCA: Hampe, 1862; Mitten, 1869 (como $P$. undulatus, n.sp.); Paris, 1906 (como P. undulatus). NORTE DE SANTANDER: Hampe, 1865b (como P. mülleri, n.sp., nom. illeg.); Mitten, 1869 (como P. mülleri). CALdAs: Mitten, 1869 (como $P$. undulatus). CaUCA, valle: Robinson, 1967.

P. dichotomus Hampe. CUNDiNAmarCA: Hampe, 1862, 1865b, n.sp.; Mitten, 1869; Paris, 1906. *SANTANDER: van der Hammen 1778 (det. Griffin).

P. fusco-lutescens Hampe. CundinamarCa: Hampe, 1865b, n.sp.; Mitten, 1869 (como P. fuscolutescens, y también como P. divaricatus, n.sp.); Paris, 1906 (como P. divaricatus); Robinson, 1967. Caldas, valle: Robinson, 1967. "Boyaca: Cleef 9496. "CaUCA: Cleef 4992. "TOlima: Cleef 3355. (det. Griffin).

P. luteo-virens (Tayl.) Mitt. SIN/LOC.: Wilson, 1847 (como Neckera). cundiNAMARCA: Hampe, 1865b (como $P$. flagellaris, n.sp.); Mitten, 1869 (como P. flagellaris, y también como $P$. laeviusculus, n.sp.). TOLIMA: Hampe, 1865 b y Mitten, 1869 (como P. flagellaris). valle: Robinson, 1967 (como P. dichotomus. Véase Griffin, 1973). *BoyACÁ: van der Hammen 2613 (det. Griffin). *MAGDalenA: Mägdefrau 1319 (det. Crum).

P. lycopodioides Hampe. CundiNAMARCA: Hampe, 1862, 1865b, n.sp.; Mitten, 1869.

P. pinnatus Hampe. CUNDiNamarCA: Hampe, 1865b, n.sp.; Mitten, 1869.

\section{Pterobryaceae}

Orthostichidium guyanense (Mont.) Broth. ANDES BOGOTENSES: Mitten, 1869 (como Meteorium excavatum, n.sp.).

Orthostichopsis auricosta (C.Müll.) Broth. PUTUMA YO: Robinson, 1967.

\section{Meteoriaceae}

Barbella tenuissima (Hook. f. \& Wils.) Fleisch. in Broth. CundinamarCa: Hampe, 1862 (como Pilotrichella); Hampe, 1865b (como Cryphaea); Mitten, 1869 (como Meteorium). 
Lindigia aciculata (Tayl.) Hampe. CUNDINAMARCA: Hampe, 1862 (como Hypnum anomalum, n.sp.); Hampe, 1865b (como L. hypnoides, nom. illeg.); Hampe, 1869.

L. debilis (Mitt.) Jaeg. CundinamarCA: Hampe, 1862 (como L. curtipes, n.sp.).

Meteoridium remotifolium (C.Müll.) Manuel. CundinamarCa: Hampe, 1862 (como Pilotrichella); 1865b (como Neckera remotifolia, y también como Neckera consimilis, n.sp.); Mitten, 1869 (como Meteorium remotifolium, y también como Meteorium consimile); Paris, 1906 (como Pilotrichella consimilis); Robinson, 1967 (como Meteoriopsis); Manuel, 1977. SIERRA NEv. SANTA MARTA, TOlima: Hampe, 1865b (como Neckera). valle: Thériot, 1937 (como Meteoriopsis remotifolia, y como Meteoriopsis consimilis); Robinson, 1967 (como Meteoriopsis); Manuel, 1977b. CaUCa, PUTUMaYo, TOlima: Robinson, 1967 (como Meteoriopsis); Manuel, 1977b. ANTIOQUIA, BOYACA, CAQUETÁ, NORTE DE SANTANDER, SANTANDER: Manuel, 1977b. *MAGDalenA: Mägdefrau 1039 (det. Crum).

Meteorium atro-luteum (C.Müll.) Jaeg. ANTIOQUiA: Müller, 1874 (como Neckera atro-lutea, n.sp.).

M. illecebrum Sull. cundinamarCa: Hampe, 1862 (como Pilotrichella fusco-viridis, nom. nud.); Hampe, 1865b (como Neckera fusco-viridis, n.sp.); Mitten, 1869 (como M. fusco-viride, y también como M. illecebrum); Paris, 1906 (como Papillaria fusco-viridis); Herzog, 1934; Robinson, 1967. valle: Robinson, 1967. *BoYACÁ: van der Hammen 1717 (det. Florschütz).

Papillaria deppei (C.Müll.) Jaeg. ANDES Bogotenses: Mitten, 1869 (como Meteorium). CUNDinamarCa, Valle: Robinson, 1967.

P. imponderosa (Tayl.) Broth. CUNDINAMARCA: Hampe, 1865b (como Lindigia pilotrichelloides, n.sp., nom. illeg.); Hampe, 1869 (como Lindigia imponderosa); Mitten, 1869 (como Meteorium); Paris, 1906 (como Lindigia); Robinson, 1967. *MAGdalena: Mägdefrau 1160 (det. Crum).

P. nigrescens (Hedw.) Jaeg. CundinamarCa: Hampe, 1865b (como Meteorium, var. gracile, n.var. y var. illecebrum, nom.nud.). SIN/LOC.: Mitten, 1869 (como Meteorium). SANTANDER' NORTE DE SANTANDER: Mitten, 1869 (como Meteorium appressum). CALDAS: Irmscher, 1914 (como P. appressa).

P. penicillata (Dozy \& Molk.) Broth. valLE: Robinson, 1967.

$P$. subsquamatula C.Müll. CUNDINAMARCA: Paris, 1906.

Pilotrichella flexilis (Hedw.) Ångstr. CUNDINAMARCA: Hampe, 1862; Hampe, 1865b (como Neckera); Mitten, 1869 (como Meteorium); Paris, 1906; Irmscher, 1914; Herzog, 1934. SANTANDER/ NORTE DE SANTANDER: Mitten, 1869 (como Meteorium). NORTE DE SANTANDER: Müller, 1857 (como Neckera turgescens); Hampe, 1865 b (como Neckera turgescens); Robinson 1967. CAUCA, VALle: Robinson, 1967. "BoYACA: van der Hammen 1713. "SANTANDER: van der Hammen 1755. (det. Florschütz). *Magdalena: Mägdefrau 1066 (det. Crum.).

P. pachygaster (C.Müll.) Jaeg. CUNDINAMARCA, MAgDALENA: Hampe, 1865b (como Neckera). —var. gracilis (C.Müll.) Par. MAGDALENA: Müller, 1857 (como Neckera, var. gracilis, n.var.).

P. pentasticha (Brid.) Wijk \& Marg. CUNDINAMARCA, PUTUMaYo, valle: Robinson, 1967. *MAGDALENA: Mägdefrau 1154 (det. Crum).

P. rigida (C.Müll.) Besch. CundinamarCa: Paris, 1906.

$P$. viridis (C.Müll.) Jaeg. MagdalEna: Mitten, 1869 (como Meteorium).

Squamidium filiferum (C.Müll.) Broth. TOLIMA: Hampe, 1865b (como Neckera). CundinamakCA: Mitten, 1869 (como Meteorium).

S. leuchotrichum (Tayl.) Broth. NORTE DE SANTANDER: Müller, 1857 (como Neckera rufescens); Hampe, 1865b (como Neckera rufescens). CundinamarCA: Hampe, 1862 (como Pilotrichella longibarbata, nom. nud.); Hampe, 1862a, 1865b (como Neckera longebarbata, n.sp.); Mitten, 1869 (como Meteorium longebarbatum); Robinson, 1967. SANTANDER/NORTE DE SANTANDER: Mitten, 1869 (como Meteorium). CALDAS, CAUCA, NORTE DE SANTANDER, PUTUMA YO, VALLE: Robinson, 1967.

S. nigricans (Hook.) Broth. NARIÑo: Kunth, 1822 (como Hypnum nigricans Hook., n.sp.); Hampe, 1865b (como Neckera); Mitten, 1869 (como Meteorium). CundinamarCa: Hampe, 1862, 1865b (como Pilotrichum macranthum); Paris, 1906 (como Pilotrichella); Herzog, 1934; Robinson, 1967. ANTIOQUIA: Müller, 1874 (como Pilotrichum sticticola, n.sp.); Irmscher, 1914. Caldas, CaUCa, PUTUmayo, valle: Robinson, 1967. *Magdalena: Mägdefrau 1153 (det. Crum).

S. ternstroemiae (Brid.) Broth. Cundinamarca: Hampe, 1865b (como Neckera). sin/Loc.: Mitten, 1869 (como Meteorium).

Zelometeorium patens (Hook.) Manuel. CAUCA/Narino: Hooker, 1818 (como Hypnum patens, n.sp.); Kunth, 1822 (como Hypnum); Hampe, 1865b (como Pilotrichum); Mitten, 1869 (como Meteorium). CUNDINAMARCA: Robinson, 1967 (como Meteoriopsis). CAQUETA: Manuel, 1977a. 
Z. patulum (Hedw.) Manuel. ANTIOQUIA: Irmscher, 1914 (como Meteoriopsis). CAUCA, CHOCÓ, CUNDinamarCa, pUtumayo, valle: Robinson, 1967 (como Meteoriopsis). AMazonas, META: Manuel, 1977a. *BOYACA: van der Hammen 1718 (det. Florschütz).

Z. recurvifolium (Hornsch.) Manuel. CHOCó, PUTUMAyo, VALLE: Robinson, 1967 (como Meteoriopsis). CAQUETA, META: Manuel, 1977a.

\section{Phyllogoniaceae}

Catagonium politum (Hook. f. \& Wils.) Broth. CundinamarCA: Mitten, 1869 (como Acrocladium); Paris, 1906 (como Acrocladium); Herzog, 1934 (como Eucatagonium).

-var. phyllogonium (C.Müll.) Card. CundinamarCA: Hampe, 1866 (como Hypnum phyllogonium).

Phyllogonium fulgens (Hedw.) Brid. TOLIMA: Kunth, 1822 (como Pterogonium). NORTE DE SANTANDER: Müller, 1857; Hampe, 1865b. CUNDINAMARCA: Hampe, 1862 var. latius, nom.nud.); Hampe, 1865b; Mitten, 1869. ANTIOQUIA: Irmscher, 1914. CAUCA, VALlE: Robinson, 1967. *CALDAS: Cleef 2565 (det. Florschütz).

P. viscosum (P.Beauv.) Mitt. CAQUeta, valle: Robinson, 1967. *CundinamarCa: Mägdefrau 1346 (det. Crum).

\section{Neckeraceae}

Calyptothecium duplicatum (Schwaegr.) Broth. SIN/LOC.: Mitten, 1869 (como Pterobryon).

C. moritzii (Hampe) Broth. CUndiNAMARCA: Hampe, 1862, 1865b (como Neckera moritzii, n.sp.).

Homaliodendron decompositum (Brid.) Wagn. CundinamarCa: Paris, 1906 (como Porotrichum).

Neckera bogotensis Mitt. Cundinamarca: Mitten, 1869, n.sp.

N. chilensis Schimp. ex Mont. Magdalena: Mitten, 1869.

N. jamesonii Tayl. CundinamarCa: Mitten, 1869.

N. lindigii Hampe. CUNdinamarCa: Hampe, 1862, 1865b, n.sp.; Mitten, 1869. BoyacÁ: Robinson, 1967. *Magdalena: Mägdefrau 1166 (det. Crum.).

N. novae-granadae C.Müll. GUAJIRA: Müller, 1857, n.sp.; Hampe, 1865b. CUNDINAMARCA: Hampe, 1862, $1865 b$.

Neckeradelphus obtusifolius (Tayl.) Steere. ANDES BOGOTENSES: Mitten, 1869 (como Neckera).

Neckeropsis disticha (Hedw.) Kindb. CundinamarCa: Hampe, 1866 (como Neckera). SANTANDER: Mitten, 1869 (como Neckera). CHOCó: Steere, 1936. valle: Thériot, 1937; Robinson, 1967. PUTUMaYo: Robinson, 1967. *MagdaleNA: Mägdefrau 1296 (det. Crum.).

N. undulata (Hedw.) Reichdt. CHOCo: Steere, 1936. PUTUMAYO: Robinson, 1967. *MaGdalENA: Mägdefrau 1248 (det. Crum.).

Porothamnium elatulum (C.Müll.) Fleisch. in Broth. ANTIOQUIA: Müller, 1874 (como Hypnum elatulum, n.sp.).

*P. expansum (Tayl.) Fleisch. in Broth. Cundinamarca: Mägdefrau 1349. MagdaLENA: Mägdefrau 1076. (det. Crum).

P. fasciculatum (Hedw.) Fleisch. CundinamarCA: Hampe, 1862, 1866 (como Hypnum); Mitten, 1869 (como Porotrichum); Paris, 1906 (como Porotrichum).

$P$. flagelliferum (Hampe) Fleisch. in Broth. CUNDINAMARCA: Hampe, 1866 (como Hypnum flagelliferum, n.sp.); Mitten, 1869 (como Porotrichum).

$P$. lancifrons (Hampe) Fleisch. in Broth. CUNDinamarCa: Hampe, 1866 (como Hypnum lancifrons, n.sp.); Mitten, 1869 (como Porotrichum).

$P$. neckeraeforme (Hampe) Herz. CUndinamaRCA: Hampe, 1866 (como Hypnum neckeraeforme, n.sp.); Mitten, 1869 (como Porotrichum). CaUCA, Valle: Robinson, 1967.

P. sparsiflorum (Hampe) Fleisch. in Broth. CUNDINAMARCA: Hampe, 1862 (como Hypnum sparsum, nom. nud.); Hampe, 1863, 1866 (como Hypnum sparsiflorum n.sp.); Mitten, 1869 (como Porotrichum).

P. substriatum (Hampe) Fleisch. in Broth. CUNDINAMARCA: Hampe, 1866 (como Neckera substriata, n.sp.); Mitten, 1869 (como Porotrichum).

Porotrichum cobanense C.Müll. VALLE: Robinson, 1967.

P. lindigii (Hampe) Mitt. CUndinamarca: Hampe, 1866 (como Pilotrichum lindigii, n.sp.); Mitten, 1869; Bescherelle, 1894 (como Pterobryon).

P. longirostre (Hook.) Mitt. CUNDiNamarCa: Mitten, 1869; Robinson, 1967. CaUCA, VALle: Robinson, 1967. *Magdalena: Mägdefrau 1307 (det. Crum). 
P. minus Hampe. CundinamarCa: Hampe, 1865b, n.sp.; Mitten, 1869.

P. mutabile Hampe. CUNDINAMARCA: Hampe, 1862, n.sp. (1862a como Neckera flabellata, nom. illeg.); Hampe, 1865b (como P. variabile, nom. nov.); Mitten, 1869 (como P. variabile); Paris, 1906 (como P. variabile).

P. plicatulum Mitt. PUTUMAYo, valle: Robinson, 1967.

P. scoposum Hampe. Cundinamarca: Hampe, 1865b, n.sp.: Mitten, 1869.

\section{Lembophyllaceae}

Porotrichondendron nitidum (Hampe) Broth. CUNDINAMARCA: Hampe, 1866 (como Pilotrichum nitidum, n.sp.); Mitten, 1869 (como Porotrichum). CALDAS/Tolima: Irmscher, 1914 (como Porotrichum).

$P$. robustum Broth. in Herz. VALLE: Robinson, 1967.

P. superbum (Tayl.) Broth. in Herz. CUNDINAMARCA: Hampe, 1862, 1865b (como Porotrichum superbum, y también como Porotrichum stolonaceum, n.sp.); Mitten, 1869 (como Porotrichum superbum y también como Porotrichum stolonaceum); Paris, 1906 (como Porotrichum superbum); Herzog, 1934; Robinson, 1967. TOLIMA: Bescherelle, 1894 (como Porotrichum stolonaceum). CAUDA, VALLE: Robinson, 1967. *BOYACÁ: van der Hammen 1967. *SANTANDER: van der Hammen 1657 (det. Florschütz).

Rigodium solutum (Tayl.) Par. CUNDinamarCA: Hampe, 1862, 1866 (como Hypnum).

R. toxarioides Broth. \& Par. CundinamarCa: Paris, 1906, n.sp. (=Oxyrrhynchium spec. fid. Broth., Nat. Pfl. 1(3): 1160. 1909).

R. toxarion (Schwaegr.) Jaeg. CUNDINAMARCA: Mitten, 1869 (como Hypnum); Paris, 1906; Herzog, 1934.

Pilotrichaceae, nom. illeg. ${ }^{3}$

Callicosta asperifolia (Mitt.) Crosby. SANTANDER: Mitten, 1869 (como Pilotrichum asperifolium, n.sp.); Crosby, 1969 (como Pilotrichum).

C. bipinnata (Schwaegr.) C.Müll. CHOCO: Steere, 1936 y Crosby, 1969 (como Pilotrichum).

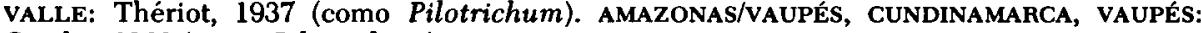
Crosby, 1969 (como Pilotrichum).

C. fendleri (C.Müll.) Crosby. ANTIOQuia, nORTe De SANTANder, putumayo: Crosby, 1969 (como Pilotrichum).

C. ramosissima (Mitt.) Crosby. Cundinamarca: Mitten, 1869 (como Pilotrichum ramosissimum, n.sp.); Crosby, 1969 (como Pilotrichum). ANTIOQUIA: Müller, 1875 (como Pilotrichum wallisii, n.sp.). valle: Robinson, 1967 (como Pilotrichum).

\section{Hookeriaceae}

Actinodontium sprucei (Mitt.) Jaeg. ANTIOQUIA: Irmscher, 1914 (como Lepidopilum).

Adelothecium bogotense (Hampe) Mitt. CundinamarCA: Hampe, 1862, 1866 (como Hookeria bogotensis, n.sp.); Mitten, 1869. valle: Robinson, 1967. *BOYACÁ: van der Hammen 1958 (det. Florschütz).

Amblytropis gemmacea (Mitt.) Broth. CundinamarCA: Mitten, 1869 (como Hookeria gemmacea, n.sp.).

A. hispidula (Mitt.) Broth. SIN/Loc.: Mitten, 1869 (como Hookeria hispidula, n.sp.).

A. ovata (Mitt.) Broth. Cundinamarca: Mitten, 1869 (como Hookeria ovata, n.sp.); Paris, 1906 (como Hookeria).

Callicostella. El nombre correcto para el género Callicostella es Schizomitrium (Crosby, 1975). Como todavia no han sido publicadas nuevas combinaciones para las especies colombianas, aquellas están referidas aquí bajo Callicostella.

C. acutifolia Thér. VALLE: Thériot, 1937, n.sp.

C. colombica Williams. valle: Williams, 1925, n.sp.

C. pallida (Hornsch.) Ångstr. ANDES BOGOTENSES: Mitten, 1869 (como Hookeria). PUTUMAYo, VALLE: Robinson, 1967.

C. plicatula Thér. valle: Thériot, 1937, n.sp.

Crossomitrium oerstedianum C.Müll. VALLE: Robinson, 1967.

C. patrisiae (Brid.) C.Müll. PUTUMAYO, valLE: Robinson, 1967.

${ }^{3}$ basado en Pilotrichum P.-Beauv., nom. illeg. (Véase Crosby, 1978). 
C. wallisii C.Müll. ANTIOQUIA: Müller, 1875, n.sp. valle: Robinson, 1967.

Calyptrochaeta. Véase Eriopus.

Cyclodictyon albicans (Hedw.) O.Kuntze. SANTANDER: Mitten, 1869 (como Hookeria). CUNDINamarCa: Paris, 1906 (como Hookeria). Cauca: Robinson, 1967.

C. capillatum (Mitt.) O.Kuntze. CundinamarCA: Mitten, 1869 (como Hookeria capillata, n.sp.).

C. cuspidatum O.Kuntze. Cundinamarca: Hampe, 1866 (como Hookeria); Mitten, 1869 (como Hookeria).

C. humile (Mitt.) O.Kuntze. SANTANDER: Mitten, 1869 (como Hookeria humilis, n.sp.). MagDALENA: Kindberg, 1901 (como Hookeria).

C. lindigianum (Hampe) O.Kuntze. CundinamarCa: Hampe, 1862, 1866 (como Hookeria lindigiana, n.sp., y var. flaccida, n. var.); Mitten, 1869 (como Hookeria).

C. nivale (C.Müll.) O.Kuntze. CundinamarCA: Hampe, 1866 (como Hookeria); Mitten, 1869 (como Hookeria).

C. obscurifolium (Mitt.) O.Kuntze. cundinamaRCA: Herzog, 1934.

C. roridum (Hampe) O.Kuntze. CundinamarCa: Hampe, 1866 (como Hookeria rorida, n.sp.); Mitten, 1869 (como Hookeria); valle: Thériot, 1937 (como C. riparium). *Magdalena: Mägdefrau 1090 (det. Crum).

C. rubrisetum (Mitt.) O.Kuntze. ANDES BOGoTENSES: Mitten, 1869 (como Hookeria rubriseta, n.sp.). CALDAS: Robinson, 1967. "CHOCÓ: Mägdefrau 1480 (det. Crum).

C. varians (Sull.) O.Kuntze. SIN/LOC.: Welch, 1971.

Daltonia bilimbata Hampe. CUNDINAMARCA: Hampe, 1862, 1865b, n.sp.; Mitten, 1869 (como D. bilimbata, y también como D. compressa, n.sp.); Herzog, 1934.

D. brevinervis Bartr. CundinamarCa: Herzog, 1934.

D. cucullata Hampe. cundinamarca: Hampe, 1865b, n.sp.; Mitten, 1869.

D. gracilis Mitt. ANTIOQuia: Müller, 1875 (como D. wallisii, n.sp.). NORTE DE SANTANDER: Müller, 1875 (como D. ocanniana, n.sp.). CUndinamaRCA, PUTUMAYO: Robinson, 1967.

D. lindigiana Hampe. CundinamarCA: Hampe, 1865b, n.sp.; Mitten, 1869 (como D. irrorata, n.sp.); Herzog, 1934.

D. longifolia Tayl. CundinamarCa: Hampe, 1865b. PUTUma Yo: Robinson, 1967. *Magdalena: Mägdefrau 1074 (det. Crum).

D. ovalis Tayl. cundinamarCa: Hampe, 1862.

D. pulvinata Mitt. CUNDINAMARCA: Mitten, 1869, n.sp.

D. tenuifolia Mitt. SIN/LOC.: Welch, 1974.

Distichophyllum elongatum Mitt. CUNDINAMARCA: Mitten, 1869, n.sp.

Eriopus. Hasta la fecha el nombre Eriopus no ha sido conservado, de manera que este género en realidad debería llamarse Calyptrochaeta (véase Margadant, 1959 y Crosby, 1974).

E. deflexus C.Müll. antioquia: Müller, 1874, n.sp.

E. nutans (Hampe) Mitt. CUNDINAMARCA: Hampe, 1862, 1865b (como Lepidopilum nutans, n.sp.); Mitten, 1869.

Helicoblepharum daltoniaceum (Hampe) Broth. Cundinamarca: Hampe, 1862 (como Lepidopilum daltoniaceum, n.sp.); Hampe, 1865b (como Daltonia binervis, nom. nov.); Mitten, 1869 (como Lepidopilum).

Hemiragis aurea (Brid.) Kindb. sin/LOc.: Welch, 1971.

Hookeriopsis acuminata (Mitt.) Jaeg. PUTUMAYO: Robinson, 1967.

H. crispa (C.Müll.) Jaeg. MAgDalena/NORTE DE SANTANDER: Müller, 1857 (como Hookeria crispa, n.sp.); Hampe, 1866 y Mitten, 1869 (como Hookeria). CundinamarCa: Hampe, 1862, 1866 (como Hookeria); Mitten, 1869 (como Hookeria). SANTANDER, SIERRA NEV. SANTA MARTA: Mitten, 1869 (como Hookeria). CAUCA: Bescherelle, 1894 (como Hookeria). valLE: Robinson, 1967.

H. cruegeriana (C.Müll.) Jaeg. CundinamarCa: Mitten, 1869 (como Hookeria). valle: Thériot, 1937; Robinson, 1967. NORTE DE SANTANDER, PUTUMAYO: Robinson, 1967.

H. diffusa (Wils.) Jaeg. CHOCó: Steere, 1936. VAlLE: Robinson, 1967.

H. falcata (Hook.) Jaeg. CAUCA/NARIÑO: Hooker, 1818 (como Hookeria falcata, n.sp.); Kunth, 1822 (idem); Hampe, 1866 (idem); Mitten, 1869 (idem). sıN/LOC.: Müller, 1857 (como Hookeria). cundinamarCa: Hampe, 1862 (como Hookeria); Mitten, 1869 (idem).

H. glandulifera (Hampe) Jaeg. CUNDINAMARCA: Hampe, 1862, 1866 (como Hookeria glandulifera, n.sp.); Mitten, 1869 (como Hookeria).

H. gracilis (Mitt.) Jaeg. CAUCA: Williams, 1908.

H. hypnacea (C.Müll.) Jaeg. CUNDINAMARCA: Hampe, 1866 (como Hookeria); Mitten, 1869 (como Hookeria). 
H. negrensis Broth. ex Thér. valLE: Thériot, 1937.

H. subfalcata (Hampe) Jaeg. CuNDINAMARCA: Hampe, 1862, 1866 (como Hookeria subfalcata, n.sp.); Mitten, 1869 (como Hookeria). CAUCA: Bescherelle, 1894 (como Hookeria). VALLE: Robinson, 1967.

H. taylorii (C.Müll.) Wijk \& Marg. CundinamanCA: Hampe, 1862, 1866 (como Hookeria). CAUCA: Bescherelle, 1894 (como Hookeria).

H. undata (Hedw.) Jaeg. SIN/LOC.: Welch, 1969.

H. variabilis (Mitt.) Jaeg. ANDES BOGOTENSES: Mitten, 1869 (como Hookeria variabilis, n.sp.).

H. velutina (Hampe) Jaeg. CUNDINAMARCA: Hampe, 1862, 1866 (como Hookeria velutina, n.sp.); Mitten, 1869 (como Hookeria).

H. viridissima (Mitt.) Jaeg. FL. MAGDalena: Mitten, 1869 (como Hookeria viridissima, n.sp.). VALLE: Robinson, 1967.

Hypnella pilifera (Hook. f. \& Wils.) Jaeg. ANDES Bogotenses: Mitten, 1869 (como Hookeria). * Magdalena: Mägdefrau 1310 (det. Crum).

Isodrepanium lentulum (Wils.) Britt. VALLE: Thériot, 1937.

Lepidopilum angustifrons Hampe. CUNDINAMARCA: Hampe, 1862, n.sp. (también como L. hyalinum, nom. nud.); Hampe, 1865b; Mitten, 1869.

L. apollinairei Broth. \& Par. CundinamarCA: Paris, 1906, n.sp.

L. aubertii Thér. VALLE: Thériot, 1937, n.sp.

L. biductulosum (P.-Beauv.) Wijk \& Marg. CHoco: Steere, 1936 (como L. flexifolium).

L. brevipes Mitt. VALLE: Robinson, 1967.

L. carneum Bartr. SIN/LOC.: Welch, 1966.

L. caudatum C.Müll. ANTIOQUiA: Müller, 1875, n.sp.

L. erubescens C.Müll. ANTIOQUiA: Müller, 1875, n.sp. VAllE: Robinson, 1967.

L. excelsum C.Müll. ANTIOQUIA: Müller, 1875, n.sp. VALLE: Robinson, 1967.

L. frondosum Mitt. SIN/LOC.: Mitten, 1869. VALLE: Thériot, 1937.

L. longifolium Hampe. CundinamarCa: Hampe, 1865b, n.sp.; Mitten, 1869.

L. maculatum C.Müll. ANTIOQUiA: Müller, 1875, n.sp.

L. mniaceum C.Müll. ANTIOQUIA: Müller, 1875, n.sp.

L. muelleri (Hampe) Spruce. CUNDinamarCa: Hampe, 1865b. Santander: Mitten, 1869.

L. pergracile C.Müll. ANTIOQUiA: Müller, 1875, n.sp.

L. perlaxum Thér. valle: Thériot, 1937, n.sp.

L. permarginatum Williams. VALlE: Williams, 1925, n.sp.

L. polytrichoides (Hedw.) Brid. SANTANDER: Mitten, 1869.

L. radicale Mitt. VALLE: Thériot, 1937.

L. robustum Mitt. CUNDINAMARCA: Mitten, 1869, n.sp.

L. scabrisetum (Schwaegr.) Steere. CUNDINAMARCA: Hampe, 1865b (como L. subenerve).

L. tortifolium Mitt. SanTander: Mitten, 1869, n.sp. PUTUMayo: Robinson, 1967.

L. wallisii C.Müll. ANTIOQUIA: Müller, 1875, n.sp.

Leskeodon andicola (Mitt.) Broth. PUTUMAYO: Robinson, 1967.

L. pusillus (Mitt.) Broth. SIN/LOC.: Mitten, 1869 (como Distichophyllum pusillum, n.sp.).

L. wallisii (C.Müll.) Broth. ex Par. ANTIOQUia: Müller, 1875 (como Mniadelphus wallisii, n.sp.). VALLE: Thériot, 1937, (fo. grandifolia).

Neohypnella diversifolia (Mitt.) Welch \& Crum. CUNDINAMARCA, VALLE: Robinson, 1967.

Schizomitrium. Véase Callicostella.

Stenodesmus latifolius Bartr. \& Herz. CundinamarCa: Herzog, 1934, n.sp.

Thamniopsis killipii (Williams) Bartr. valle: Williams, 1925 (como Hookeriopsis killipii, n.sp.).

T. pendula (Hook.) Fleisch. VALle: Robinson, 1967 (como Hookeriopsis).

\section{Leucomiaceae}

Leucomium acrophyllum (Hampe) Mitt. CUNDINAMARCA: Hampe, 1866 (como Hypnum acrophyllum, n.sp.); Mitten, 1869.

L. compressum Mitt. PUTUMAYO: Robinson, 1967.

L. lignicola Spruce ex Mitt. PUTUMayo: Robinson, 1967.

Hypopterygiaceae

Hypopterygium rigidulum Mitt. ANDES BOGOTENSES: Mitten, 1869, n.sp.

H. tamariscinum (Hedw.) Brid. CundinamarCA: Hampe, 1862, 1866 (como H. scutellatum); Mitten, 1869. CAUCA: Bescherelle, 1894 (como H. scutellatum). valle: Robinson, 1967. 
Fabroniaceae

Fabronia lindigiana Hampe. cundinamarCA: Hampe, 1862, 1865b, n.sp.; Mitten, 1869.

F. polycarpa Hook. TOLIMA: Hooker, 1818; Kunth, 1822. CUNDINAMARCA, SANTANDER: Hampe, $1862,1865 \mathrm{~b}$.

\section{Leskeaceae}

Leskea angustata Tayl. CUNDINAMARCA: Hampe, 1862, 1865b (como Anomodon); Mitten, 1869 (como L. gracillima).

\section{Thuidiaceae}

Thuidium arzobispoae (C.Müll.) Jaeg. CUndinamarCA: Hampe, 1862, 1866 (como Hypnum intermedium, hom. illeg.); Müller, 1875 (como Hypnum arzobispoae, n.sp.).

T. carantae (C.Müll.) Jaeg. ANTIOQUIA: Müller, 1875 (como Hypnum carantae, n.sp.).

T. ciliatum Mitt. MAGDALENA: Kindberg, 1901.

T. complanum Mitt. MAGDALENA: Kindberg, 1901.

T. frontinoae (C.Müll.) Jaeg. ANTiOQUiA: Müller, 1875 (como Hypnum frontinoae, n.sp.).

T. gratum (P.-Beauv.) Jaeg. QUINDIO/TOLIMA: Kunth, 1822 (como Hypnum).

T. involvens (Hedw.) Mitt. QUINDIO/TOLIMA: Kunth, 1822 (como Leskea). MAGDALENA: Kindberg, 1901 .

T. minutulum (Hedw.) B.S.G. CUNDINAMARCA: Hampe, 1866 (como Hypnum pauperum). SANTANDER: Hampe, 1866 (como Hypnum muriculatum, n.sp., hom. illeg.).

T. peruvianum Mitt. CUNDINAMARCA: Mitten, 1869, n.sp.; Herzog, 1934; Robinson, 1967. BoYACA, CALDAS, NARIÑo, SANTANDER, TOLIMA, VAlle: Robinson, 1967.

T. pseudo-delicatulum Jeg. ANTIOQUIA: Müller, 1875 (como Hypnum pseudo-delicatulum, n.sp., hom. illeg.).

T. pseudo-protensum (C.Müll.) Mitt. CundinamarCa: Hampe, 1862 (como Hypnum).

T. recognitum (Hedw.) Lindb.

- subsp. philibertii (Limpr.) Dix. CundinamarCa: Cleef 792 (det. Florschütz).

-var. delicatulum (Hedw.) Warnst. cundinAmarCA: Hampe, 1866 (como Hypnum delicatulum); Mitten, 1869 (como T. delicatulum); Paris, 1906 (como T. delicatulum); Irmscher, 1914 (como T. delicatulum). TOLIMA: Robinson, 1967 (como T. delicatulum). *BOYACA: van der Hammen 2193. *SANTANDER: van der Hammen 1729. (det. Florschütz). *MAGDALENA: Mägdefrau 1042 (det. Crum).

T. tamariscinum (Hedw.) B.S.G. CUNDINAMARCA: Hampe, 1866 (como Hypnum).

T. urceolatum Lor. CUNDINAMARCA: Robinson, 1967 (como T. antillarum). *MAGDALENA: Mägdefrau 1262 (det. Crum).

\section{Amblystegiaceae}

Calliergon cuspidatum (Hedw.) Kindb. BOYACA: Robinson, 1967 (como Calliergonella). *META: Cleef 859 (det. Ando).

${ }^{*}$ C. sarmentosum (Wahlenb.) Kindb. CAUCA: Cleef 624a (det. Florschütz).

Campylium hispidulum (Brid.) Mitt. CUNDINAMARCA: Hampe, 1866 (como Hypnum); Mitten, 1869.

Cratoneuron filicinum (Hedw.) Spruce. CundinamarCa: Robinson, 1967.

Drepanocladus exannulatus (B.S.G.) Warnst. VALLE: Robinson, 1967.

D. fluitans (Hedw.) Warnst. CUNDINAMARCA: Robinson, 1967.

*D. revolvens (Sw.) Warnst. MAgdalena: Mägdefrau 1240 (det. Crum).

Hygrohypnum pelichucense Williams. CUNDINAMARCA: Robinson, 1967.

H. tequendamense Herz. CUNDINAMARCA: Herzog, 1949, n.sp. -var. subfalcatum Herz. CUNDINAMARCA: Herzog, 1949, n.var.

Platyhypnidium. Véase Brachytheciaceae.

Sciaromium crassinervatum Mitt. CUndinamaRCA: Mitten, 1869.

\section{Brachytheciaceae}

Brachythecium asperulum Jaeg. CUNDINAMARCA: Hampe, 1862, 1866 (como Hypnum asperulum, n.sp., hom. illeg.).

B. chrysocladon (Hampe) Jaeg. Cundinamarca: Hampe, 1866 (como Hypnum chrysocladon, n.sp.); Mitten, 1869 (como Hypnum). 
B. conostomum (Tayl.) Jaeg. NORTE DE SANTANDER: Robinson, 1967.

B. flexiventrosum (C.Müll.) Jaeg. CAUCA, CUNDINAMARCA, TOLIMA, VALLE: Robinson, 1967.

B. laevifolium (Mitt.) Jaeg. CUNDINAMARCA: Mitten, 1869 (como Hypnum laevifolium, n.sp.).

B. occidentale (Hampe) Jaeg. CUNDINAMARCA: Hampe, 1866 (como Hypnum occidentale, n.sp.); Mitten, 1869 (como Hypnum); Robinson, 1967.

B. plumosum (Hedw.) B.S.G. CUNDinamarCa: Mitten, 1869 (como Hypnum). Magdalena: Robinson, 1967.

B. prasophyllum (Hampe) Jaeg. BOYACÁ, CUNDINAMARCA: Hampe, 1866 (como Hypnum prasophyllum n.sp.); Mitten, 1869 (como Hypnum); Robinson, 1967. CALDAS, CAUCA, NORTE DE SANTANDER, TOLIMA: Robinson, 1967.

B. pseudo-rutabulum (Hampe) Jaeg. CUNDinamarCA: Hampe, 1862a, 1866 (como Hypnum pseudo-rutabulum, n.sp.); Mitten, 1869 (como Hypnum); Paris, 1906. CalDas: Robinson, 1967.

B. rutabulum (Hedw.) B.S.G. CUNDINAMARCA: Robinson, 1967.

B. stereopoma (Mitt.) Jaeg. ANTIOQUIA: Irmscher, 1914. CUNDiNAMARCA: Herzog, 1934.

Camptothecium pseudo-lutescens (Hampe) Jaeg. CUNDINAMARCA: Hampe, 1866 (como Hypnum pseudo-lutescens, n.sp.); Mitten, 1869 (como Hypnum).

Eurhynchiella semitorta (Sauerb.) Broth. CUNDINAMARCA: Mitten, 1869 (como Hypnum semitortum, n.sp., hom. illeg.).

Eurhynchium oedogonium (C.Müll.) Broth. CUndinamarCA: Herzog, 1934.

E. pseudo-piliferum (Hampe) Sauerb. Cundinamarca: Hampe, 1866 (como Hypnum pseudopiliferum, n.sp.); Mitten, 1869 (como Hypnum).

E. pulchellum (Hedw.) Jenn. CUNDINAMARCA: Robinson, 1967 (como Rhynchostegium).

E. semiscabrum Bartr. CUNDINAMARCA: Robinson, 1967 (como Rhynchostegium).

Homalothecium leskeoides (Hook.) H.Robins. CundinamarCa: Hampe, 1865b (como Leskea bonplandii); Mitten, 1869 (como Hypnum bonplandii); Herzog, 1934 (como Pleuropus bonplandii). CAUCA: Robinson, 1967.

Oxyrrhynchium praelongum (Hedw.) Warnst. CUndinAmarCA: Hampe, 1862, 1866 (como Hypnum exasperatum, n.sp.); Mitten, 1869 (como Hypnum praelongum); Robinson, 1967 (como Stokesiella). *MAGDALENA: Mägdefrau 1101 (det. Crum).

O. remotifolium (Grev.) Broth. CUNDINAMARCA: Hampe, 1862, 1866 (como Hypnum clinocarpum). TOLIMA: Robinson, 1967 (como Rhynchostegium).

O. rugisetum (Hampe) Broth. ex Par. CundinamarCa: Hampe, 1866 (como Hypnum rugisetum, n.sp.).

Platyhypnidium aquaticum (Sauerb.) Fleisch. CUNDINAMARCA: Hampe, 1862, 1866 (como Hypnum aquaticum, n.sp., hom. illeg.); Mitten, 1869 (como Hypnum); Thériot, 1906 (como Rhynchostegium); Herzog, 1934. SANTANDER/NORTE DE SANTANDER: Mitten, 1869 (como Hypnum).

P. riparioides (Hedw.) Dix. CAUCA, CUNDINAMARCa: Robinson, 1967 (como Rhynchostegium). *MAGDalena: Mägdefrau 1255 (det. Crum).

Rhynchostegiella attenuata Bartr. PUTUMAYO, TOLIMA: Robinson, 1967.

Rhynchostegium chrysophylloides Sauerb. in Jaeg. CUnDINAMARCA: Hampe, 1869 (como Hypnum chrysophylloides, n.sp., hom. illeg.).

R. conchophyllum (Tayl.) Sauerb. in Jaeg. CundinamarCa: Hampe, 1862, 1866 (como Hypnum). CAUCA: Williams, 1908.

R. scariosum (Tayl.) Jaeg. CUNDinamanCA: Hampe, 1862, 1866 (como Hypnum); Mitten, 1869 (como Hypnum); Robinson, 1967. ANTIOQUIA: Irmscher, 1914. VAlle: Robinson, 1967. *BOYACÁ: van der Hammen 2694 (det. Florschütz).

R. subrotundum (Hampe) Sauerb. in Jaeg. CUNDINAMARCA: Hampe, 1866 (como Hypnum subrotundum, n.sp.); Mitten, 1869 (como Hypnum).

$R$. tocaremae (Hampe) Sauerb. in Jaeg. CUnDinamarCA: Hampe, 1866 (como Hypnum tocaremae, n.sp.); Mitten, 1869 (como Hypnum).

R. ulicon (Tayl.) Sauerb. in Jaeg. CUnDINAMARCA: Hampe, 1866 (como Hypnum); Mitten, 1869 (como Hypnum).

\section{Entodontaceae}

Entodon aurescens Hampe. CundinamarCA: Hampe, 1865b, n.sp.; Mitten, 1869.

E. beyrichii (Schwaegr.) C.Müll. MAGDALENA: Kindberg, 1901.

E. complicatus (C.Müll.) Mitt. MaGdalena: Kindberg, 1901.

E. cupressiformis Hampe. CUndinamaRCA: Hampe, 1865b, n.sp.; Mitten, 1869. 
E. gracilisetus Hampe. CUndinamarCA: Hampe, 1865b, n.sp.; Mitten, 1869.

E. jamesonii (Tayl.) Mitt. CUNDINAMARCA: Hampe, 1865 b (como E. lutescens, n.sp., y también como E. polycarpus, n.sp.); Mitten, 1869; Thériot, 1906; Robinson, 1967.

E. macropodus (Hedw.) C.Müll. MAGDalena: Mitten, 1869. SANTANDER: Iwatsuki \& Sharp, 1967.

E. propinquus Hampe. CundinamarCa: Hampe, 1865b, n.sp.; Mitten, 1869.

E. ramosissimus Hampe. CundiNAMARCA: Hampe, 1865b, n.sp.; Mitten, 1869.

Erythrodontium consanguineum (Hampe) Par. CUNDINAMarCa, SANTANDER: Hampe, 1865b (como Leptohymenium consanguineum, n.sp.); Hampe, 1869 (como Pterigynandrum); Mitten, 1869 (como Entodon). CUNDINAMARCa: Irmscher, 1914.

E. longisetum (Hook.) Par. QUINDIO/TOLIMA: Hooker, 1818 (como Neckera longiseta, n.sp.); Kunth, 1822 (como Neckera); Mitten, 1869 (como Entodon). SIERRA NEv. SANTA MARTA: Müller, 1857 (como Neckera cylindricaulis); Mitten, 1869 (como Entodon). CUNDINAMARCA: Hampe, 1862, 1865b (como Leptohymenium longisetum, y también como Leptohymenium cylindricaule); Hampe, 1869 (como Pterigynandrum longisetum, y también como Pterigynandrum cylindricaule); Mitten, 1869 (como Entodon); Bescherelle, 1894 (como Entodon). CAUCA: Bescherelle, 1894 (como Entodon); Robinson, 1967. ANTIOQUIA: Irmscher, 1914. -var. rupestre (Hampe) Wijk \& Marg. CUNDINAMARCA: Hampe, 1865b (como Leptohymenium cylindricaule var. rupestris, n.var.).

E. squarrosum (Hampe) Par. CUNDINAMARCA: Hampe, 1862, 1865b (como Leptohymenium squarrosum, n.sp.); Hampe, 1869 (como Pterigynandrum); Mitten, 1869 (como Entodon).

Mesonodon onustus Hampe. SANTANDER: Hampe, 1865b, n.sp.; Mitten, 1869 (como Entodon). CAUCA: Bescherelle, 1894.

Pleurozium schreberi (Brid.) Mitt. CundinamarCa: Hampe, 1866 (como Hypnum); Mitten, 1869; Paris, 1906 (como Hypnum); Herzog, 1934; Robinson, 1967. CAUCA, TOLIMA: Robinson, 1967.

\section{Plagiotheciaceae}

Isopterygium chrismarii (C.Müll.) Mitt. META, VALLE: Robinson, 1967.

l. deplanatiforme (Kindb.) Broth. MAgDalENA: Kindberg, 1901 (como Rhynchostegium deplanatiforme, n.sp.).

I. diminutivum Bart. PUTUMAyo: Robinson, 1967.

I. hospitans (Hampe) Jaeg. CUNDINAMARCA: Hampe, 1866 (como Hypnum hospitans, n.sp.).

I. pseudo-tenerum (Broth. \& Kindb.) Broth. MAgDALENA: Kindberg, 1901 (como Plagiothecium pseudo-tenerum, n.sp.).

I. tenerum (Sw.) Mitt. CUNDINAMARCA: Hampe, 1862, 1866 (como Hypnum pygmaeum). FL. MAGDalena: Mitten, 1869. Magdalena: Kindberg, 1901 (como Plagiothecium). aNTIOQUia: Irmscher, 1914.

Pilosium chlorophyllum (Hornsch.) C.Müll. PUTUMayo: Robinson, 1967.

Plagiothecium andinum (Hampe) Schimp. in Jaeg. BOYACÁ, CUNDINAMARCA: Hampe, 1866 (como Hypnum andinum, n.sp.).

P. denticulatum (Hedw.) B.S.G. VALLE: Robinson, 1967.

P. lucidulum (Hook. f. \& Wils.) Mitt. CundinamarCa: Herzog, 1934.

P. novo-granatense (Hampe) Mitt. CUNDINAMARCA: Hampe, 1862, 1866 (como Hypnum novogranatense, n.sp.); Mitten, 1869.

${ }^{*} P$. roeseanum B.S.G. CUNDiNAMARCA: Mägdefrau 1560 (det. Crum).

\section{Sematophyllaceae}

Acroporium pungens (Hedw.) Broth. SANTANDER: Mitten, 1869 (como Sematophyllum). cHoco: Steere, 1936. valle: Thériot, 1937; Robinson, 1967. NORTE DE SANTANDER: Robinson, 1967. Aptychella colombica Williams. SANTANDER: Williams, 1930, n.sp.

A. proligera (Broth.) Herz. CundinamarCA: Herzog, 1934 (fo. filiramea); Robinson, 1967. SANTANDER: Robinson, 1967.

Aptychopsis pycnodonta Herz. Cundinamarca: Herzog, 1934, n.sp.

Glossadelphus laevifolius (Mitt.) Bartr. ANDES BOGOTENSES: Mitten, 1869 (como Ectropothecium laevifolium, n.sp.).

G. truncatulus (C.Müll.) Fleisch. PUTUMayo: Robinson, 1967.

Heterophyllium nemorosum (Brid.) Kindb. TOLIMA: Kunth, 1822 (como Hypnum affine Hook., n.sp., hom. illeg.); Hampe, 1866 (como Hypnum affine); Mitten, 1869 (como Stereodon af- 
finis). Cundinamarca: Hampe, 1862, 1866 (como Hypnum affine); Mitten, 1869 (como Stereodon affinis). VALLE: Robinson, 1967. *BOYACÁ: van der Hammen 1950. *SANTANDER: van der Hammen 1724. (det. Florschütz).

Pterogonidium pulchellum (Hook.) C.Müll. in Broth. TolıMA: Hooker, 1818 (como Pterogonium pulchellum, n.sp.); Kunth, 1822 (como Pterogonium); Hampe, 1865b (como Pterogonium); Mitten, 1869 (como Potamium). PUTUMayo: Robinson, 1967.

Rhaphidorrhynchitu decumbens (Mitt.) Broth. CUNDINAMARCA: Mitten, 1869 (como Sematophyllum decumbens, n.sp.); Herzog, 1934.

R. erythropodium (Hampe) Broth. CUNDINAMARCA: Mitten, 1869 (como Sematophyllum erythropodium, n.sp.).

R. fragilirostrum (Hampe) Broth. CundinamaRCA: Hampe, 1862, 1866 (como Hypnum fragilirostrum, n.sp.); Mitten, 1869 (como Sematophyllum).

R. lindigii (Hampe) Broth. CundiNamarCa: Hampe, 1862, 1866 (como Hypnum lindigii, n.sp.); Mitten, 1869 (como Sematophyllum). valle: Robinson, 1967 (como Sematophyllum). *BOYACÁ: van der Hammen 2709 (det. Florschütz). *Magdalena: Mägdefrau 1317 (det. Crum).

R. roridum (Mitt.) Broth. CUNDINAMARCA: Mitten, 1869 (como Sematophyllum roridum, n.sp.).

R. schlimii (C.Müll.) Broth. CUNDINAMARCA: Hampe, 1866 (como Hypnum,-var. minor, noin. nud.).

R. sticticola (C.Müll.) Broth. ANTIOQUIA: Müller, 1874 (como Hypnum sticticola, n.sp.).

R. subscabrum (C.Müll.) Broth. CundinamarCA: Hampe, 1862, 1866 (como Hypnum); Mitten, 1869 (como Sematophyllum).

Sematophyllum aureo-sulfureum (C.Müll.) Broth. ANTIOQUiA: Müller, 1874 (como Hypnum aureo-sulfureum, n.sp.).

S. caespitosum (Hedw.) Mitt. Cundinamarca: Hampe, 1862, 1866 (como Hypnum); Hampe, 1866 (también como Hypnum agnatum, n.sp., como Hypnum cucullatifolium, n.sp., y como Hypnum kegelianum var. latior y angustior, nom. nud.); Mitten, 1869 (como S. agnatum, y también como S. cucullatifolium); Herzog, 1934 (como S. cucullatifolium); Robinson, 1967. SANTANDER: Hampe, 1866 (como Hypnum cespitosum, y también como Hypnum cucullatifolium, y como Hypnum loxense). sIN/LOC.: Mitten, 1869 (como Sematophyllum leptothecium, n.sp.). NARIÑo: Bescherelle, 1894 (como S. loxense). MAGDALENA: Kindberg, 1901 (como Rhaphidostegium subloxense, n.sp.). ANTIOQUIA: Irmscher, 1914 (como Rhaphidostegium loxense). CHOCo: Steere, 1936. PUTUMAYO, TOLIMA: Robinson, 1967.

S. chrysostegum (C.Müll.) Mitt. SANTANDER: Hampe, 1869 (como Hypnum).

S. crassiusculum (Brid.) Broth. TOLIMA: Kunth, 1822 (como Hypnum).

S. flavidum Mitt. Cundinamarca: Mitten, 1869, n.sp. Valle: Robinson, 1967. *Magdalena: Mägdefrau 1130 (det. Crum).

S. galipense (C.Müll.) Mitt. CundinamarCa: Mitten, 1869. Magdalena: Kindberg, 1901 (como Rhaphidostegium). VALlE: Robinson, 1967.

S. pennellii Williams. CAUCA: Williams, 1925, n.sp.

S. subbrachycarpum (Hampe) Mitt. CUNDINAMARCA, SANTANDER: Hampe, 1866 (como Hypnum subbrachycarpum, n.sp.); Mitten, 1869.

S. subsimplex (Hedw.) Mitt. SIN/Loc.: Mitten, 1869 (fo. minor). MAGDalena: Kindberg, 1901 (como Rhaphidostegium). CAUCA: (?) Williams, 1908. PUTUMAYO, VALLE, VAUPÉS: Robinson, 1967.

S. tequendamense (Hampe) Mitt. CUNDINAMARCA: Hampe, 1862, 1866 (como Hypnum tequendamense, n.sp. y como Hypnum crithmifolium, n.sp.); Mitten, 1869 (como S. tequendamense y como S. crithmifolium). NARIÑo: Bescherelle, 1894.

Taxithelium planum (Brid.) Mitt. SANTANDER, viCHADA: Mitten, 1869. Chocó: Steere, 1936. PUTUMAYO, VALLE: Robinson, 1967.

Trichosteleum cyparissoides (Hornsch.) H.Robins. VALLE: Robinson, 1967.

T. fluviale (Mitt.) Sauerb. in Jaeg. FL. MAGDALENA: Mitten, 1869 (como Sematophyllum fluviale, n.sp.). PUTUMAYO: Robinson, 1967.

T. papillosum (Hornsch.) Sauerb. in Jaeg. VALLE: Thériot, 1937

Wijkia subnitida (Hampe) Crum. CUNDINAMARCA: Hampe, 1866 (como Hypnum subnitidum, n.sp.).

Hypnaceae

Ctenidium malacodes Mitt. CUndinamarCa: Mitten, 1869, n.sp.; Robinson, 1967. CALDAS, PUTUMAYO, VALlE: Robinson, 1967.

Ectropthecium campanulatum Mitt. CUNDINAMARCA: Mitten, 1869, n.sp.; Paris, 1906. 
E. globitheca (C.Müll.) Mitt. Sierra NEV. SANTA MARTA: Müller, 1857 y Hampe, 1866 (como Hypnum). CUNdiNamarca: Hampe, 1862 (como Hypnum apiculatum); Hampe, 1866 (como Hypnum); Mitten, 1869 (como E. apiculatum , como E. globitheca); Paris, 1906 (como E. apiculatum). PUTUMAYo: Robinson, 1967 (como E. apiculatum). *MAGdalena: Mägdefrau 1147 (det. Crum).

Hypnum amabile (Mitt.) Hampe. CundinamarCA: Hampe, 1866 (como H. subimponens, n.sp., hom. illeg.); Mitten, 1869 (como Ectropothecium amabile, n.sp.); Hampe, 1869; Paris, 1906 (como Ectropothecium); Irmscher, 1914 (como Stereodon); Herzog, 1934; Robinson, 1967. BOYACA: Robinson, 1967.

H. cupressiforme Hedw. cundinamarca: Hampe, 1866; Paris, 1906.

H. hamatum (Mitt.) Sauerb. in Jaeg. (hom. illeg.) CUNDINAMARCA: Mitten, 1869 (como Stereodon hamatus n.sp.); Robinson, 1967 (como Stereodon).

H. spiripes C.Müll. sıN/LOC.: Müller, 1857, n.sp.; Hampe, 1866; Mitten, 1869 (como Stereodon). Isopterygium. Véase Plagiotheciaceae.

Microthamnium pelichucense "(Williams)," nom. nud. CUNDINAMARCA: Herzog, 1934; probablemente basado en un nombre preliminar en el Herbario Williams y posiblemente idéntico con Hygrohypnum pelichucense Williams.

M. smithii Williams, hom. illeg. SANTANDER: Williams, 1930, n.sp.

Mittenothamnium andicola (Hook.) Card. CALDAS, CUndinamarCA: Mitten, 1869 (como Microthamnium).

M. diminutivum (Hampe) Britt. CUNDINAMARCA: Hampe, 1862, 1866 (como Hypnum perspicuum, n.sp.); Hampe, 1866 (también como Hypnum thelistegum). SIERRA NEv. SANTA MARTA: Hampe, 1866 (como Hypnum perspicuum). SANTANDER: Mitten, 1869 (como Microthamnium thelistegum). MAGDALENA: Kindberg, 1901 (como Microthamnium thelistegum). *BOYACA: van der Hammen 2695 (det. Florschütz).

—var. molle (Hampe) Wijk \& Marg. CundinamarCA: Hampe, 1866 (como Hypnum perspicuum var. molle, n.var.).

—var. teres (Hampe) Wijk \& Marg. CundinamarCa: Hampe, 1866 (como Hypnum perspicuum var. teres, n.var.).

M. langsdorffi (Hook.) Card. vicHADA: Kunth, 1822 (como Hypnum langsdorffi Hook., n.sp.). Sin/LOC.: Mitten, 1869 (como Microthamnium). CundinamarCa: Herzog, 1934 (como Microthamnium); Robinson, 1967.

M. lehmannii (Besch.) Card. valle: Robinson, 1967.

M. loriforme (Hampe) Card. CundinamarCa: Hampe, 1862, 1866 (como Hypnum loriforme, n.sp.); Mitten, 1869 (como Microthamnium).

M. reptans (Hedw.) Card. CAUCA: Williams, 1908 (como Microthamnium). CAUCA: Williams, 1908 (como Microthamnium tuerckheimii). CUNDINAMARCA, VALLE: Robinson, 1967. *BOYACA: van der Hammen 2744. *SANTANDER: van der Hammen 1735. (det. Florschütz). *MAGDALENA: Mägdefrau 1078 (det. Crum).

M. subobscurum (Hampe) Card. CUNDINAMARca: Hampe, 1866 (como Hypnum subobscurum, n.sp.).

M. substriatum (Mitt.) Card. CUNDINAMARCA: Mitten, 1869 (como Microthamnium substriatum, n.sp.).

M. viscidulum (Hampe) Card. CUNDINAMARCA: Hampe, 1862, 1866 (como Hypnum viscidulum, n.sp.); Hampe, 1866 var. gracilitus, nom. nud.); Mitten, 1869 (como Microthamnium). SANTANDER: Mitten, 1869 (como Microthamnium).

M. volvatum (Hampe) Card. CUNDINAMARCA: Hampe, 1862, 1866 (como Hypnum volvatum, n.sp.); Paris, 1906 (como Microthamnium).

-var. gracilius (Hampe) Wijk \& Marg. CUNDINAMARCA: Hampe, 1866 (como Hypnum volvatum var. gracilius, n.var.).

*Pylaisiella falcata (B.S.G.) Ando. CundinamarCa: Cleef 6035 (det. Ando).

Vesicularia amphibola (Mitt.) Broth. SIN/LOC.: Mitten, 1869 (como Ectropothecium amphibolum, n.sp.). CHOCó: Steere, 1936. PUTUMAYO, TOLIMA: Robinson, 1967.

V. poeppigiana (Hampe) Crum \& Steere. Fl. MaGdalena: Hampe, 1866 (como Hypnum).

\section{Rhytidiaceae}

Rhytidiadelphus novogranatensis Williams. SANTANDER: Williams, 1930, n.sp.

\section{Polytrichaceae}

Atrichum androgynum (C.Müll.) Jaeg. CAUCA: Bescherelle, 1894 (como A. bogotense, n.sp.). *A. oerstedianum (C.Müll.) Mitt. MagDalenA: Mägdefrau 1253 (det Crum). 
A. polycarpum (C.Müll.) Mitt. CUNDINAMARCA, BOYACA: Hampe, 1862, 1865b (como Catharinea); Mitten, 1869; Herzog, 1934 (como Catharinea). ANTIOQUIA: Irmscher, 1914 (como Catharinea). *SANTANDER: van der Hammen 1728 (det. Florschütz).

Notoligotrichum trichodon (Hook. f. \& Wils.) G.L.Smith. CALDAS/TOLIMA: Wilson, 1847 (como Polytrichum trichodon, n.sp.); Hampe, 1865b (como Catharinea); Mitten, 1869 (como Psilopilum).

Oligotrichum erosum (Hampe) Lindb. CundinamaRCA: Hampe, 1862, 1865b (como Catharinea erosa, n.sp.); Mitten, 1869. SIN/LOC.: Mullen \& Frye, 1947. CAL.DAS: Robinson, 1967.

Pogonatum abbreviatum Mitt. SANTANDER: Mitten, 1869, n.sp.

$P$. andinum (Hampe) Mitt. CUndiNAMARCA: Hampe, 1862, 1865 b (como Polytrichum andinum, n.sp.); Mitten, 1869; Herzog, 1934; Robinson, 1967.

P. arcuatum Mitt. CUNDINAMARCA: Mitten, 1869, n.sp.; Robinson, 1967.

P. cuspidatum Besch. CundinamarCa: Robinson, 1967. *MAgdalena: Mägdefrau 1185 (det. Crum).

P. neglectum (Hampe) Jaeg. CUndinamarCa: Hampe, 1869 (como Polytrichum neglectum, n.sp.). ANTIOQUIA: Irmscher, 1914. CAQUETA: Robinson, 1967. *MAGDALENA: Mägdefrau 1035 (det. Crum).

P. oligodus (C.Müll.) Mitt. Cundinamarca: Hampe, 1862, 1865b (como Polytrichum jamesonii); Hampe, 1865b (como Polytrichum cucullatum, n.sp.); Mitten, 1869 (como P. cucullatum, y como P. oligodus). CAUCA: Bescherelle, 1894. BOYACÁ, vaLLE: Robinson, 1967.

P. psilopiloides (C.Müll.) Par. ANTIOQUia: Müller, 1874 (como Polytrichum psilopiloides, n.sp.).

P. purpurascens Mitt. CUNDINAMARCA: Hampe, 1862, 1865b (como Polytrichum purpurascens, n.sp., hom. illeg.); Mitten, 1869; Paris, 1906.

P. semipellucidum (Hampe) Mitt. QUINDIO/TOLIMA: Kunth, 1822 (como Polytrichum convolutum); Hampe, 1865b (como Polytrichum); Mitten, 1869. NORTE DE SANTANDER: Müller, 1857 (como Polytrichum); Hampe, 1865b (como Polytrichum); Mitten, 1869. Cundinamarca: Hampe, 1862, 1865b (como Polytrichum); Mitten, 1869 (también como P. viride, n.sp.). Loc. IGNOT. (Osson): Mitten, 1869 (como P. subbifarium, n.sp.). valle: Robinson, 1967.

P. subcontortum (Hampe) Mitt. CUNDINAMARCA: Hampe, 1865b (como Polytrichum subcontortum, n.sp.); Mitten, 1869.

P. tortile (Sw.) Brid. CaUCA: Williams, 1908. Magdalena: Robinson, 1967.

P. varians (Hampe) Mitt. CUndinamarca: Hampe, 1865b (como Polytrichum varians, n.sp.); Mitten, 1869.

Polytrichadelphus abriaquiae (C.Müll.) Sauerb. in Jaeg. ANTIOQuiA: Müller, 1875 (como Catharinea abriaquiae, n.sp.).

P. aristatus (Hampe) Mitt. Cundinamarca: Hampe, 1862, 1865b (como Catharinea aristata, n.sp.); Mitten, 1869; Paris, 1906. NORTE DE SANTANDER: Bartram, 1953.

P. ciliatus (Hook. f. \& Wils.) Mitt. NORTE DE SANTANDER: Wilson, 1847 (como Polytrichum ciliatum, n.sp.); Müller, 1848 (como Catharinea); Hampe, 1865b (como Catharinea); Mitten, 1869. NARIÑo: Bescherelle, 1894. CAUCA: Williams, 1908. CALDAS: Robinson, 1967.

P. giganteus (Hook.) Mitt. QUINDIO/TOLIMA: Hooker, 1818 (como Polytrichum giganteum, n.sp.); Kunth, 1822 (como Polytrichum); Mitten, 1869.

P. longisetus (Brid.) Mitt. QUINDIO/TOLIMA: Hooker, 1818 (como Polytrichum longisetum, n.sp., hom. illeg.); Kunth, 1822 (como Polytrichum); Mitten, 1869. Tolima: Müller, 1857 (como Polytrichum trianae, n.sp.); Hampe, 1865b (como Catharinea trianae); Mitten, 1869 (como P. trianae). ANTIOQUIA: Irmscher, 1914 (como P. trianae). CALDAS, CUNDINAMARCA, PUTUMAYO: Robinson, 1967.

P. purpureus Mitt. CALDAS, CAUCA, TOLIMA: Robinson, 1967.

P. rubiginosus Mitt. CUNDINAMARCA: Mitten, 1869, n.sp.

Polytrichastrum tenellum (C.Müll.) G.L.Smith. CUNDINAMARCA: Robinson, 1967 (como Pogonatum alpiniforme); Smith, 1975. * MAGDaleNA: Mägdefrau 1105 (det. Crum).

Polytrichum commune Hedw. BoyaCÁ, Cundinamarca: Robinson, 1967.

P. ericoides Hampe, hom. illeg. CundinamarCA: Hampe, 1865b; Mitten, 1869 (como Polytrichadelphus); Robinson, 1967 (como Polytrichadelphus); Smith, 1976. TOLIMA: Robinson, 1967 (como Polytrichadelphus). BOYACA: Smith, 1976.

P. juniperinum Willd. ex Hedw. CUNDINAMARCA, BOYACA: Hampe, 1862, 1865b; Hampe, 1865b (también como $P$. antillarum); Herzog, 1934 (como $P$. antillarum, subsp. substrictum); Mitten, 1869 (como P. aristiflorum n.sp.); Paris, 1906 (como P. aristiflorum); Irmscher, 1914 (como P. aristiflorum); Robinson, 1967. ANTIOQUIA: Müller, 1874 (como P. equisetiforme, n.sp., como $P$. rhynchomitrium, n.sp., y como $P$. thysanomitrium n.sp.). CALDAS, TOLIMA: Irmscher, 1914 (como P. aristiflorum). Magdalena: Messmer \& Frye, 1947. CAQUeTÁ, CaUCA, PUTUMAYo, SANTANDER, VALlE: Robinson, 1967. *arauca: Cleef 8840 (det. Florschütz). 


\section{SINÓNIMOS}

Cambios de nomenclatura de acuerdo con el Index Muscorum y revisiones taxonómicas subsiguientes. La indicación del nombre solo de un género en esta lista implica que el epiteto específico original fué transferido a ese género.

Acrocladium politum (Hook.f. \& Wils.) Mitt. = Catagonium.

Amphoritheca jamesonii (Tayl.) Hampe = Funaria.

A. lindigii Hampe $=$ Funaria .

A. lutescens Hampe = Funaria.

A. pseudo-marginata Hampe = Funaria trumpffi.

A. undulata Hampe = Funaria.

Anacalypta cucullata Hampe $=$ Pottia.

A. subcaespitosa Hampe = Barbula.

Anacolia subsessilis (Tayl.) Broth. = A. laevisphaera.

Andreaea aristata Herz. = A. subulata (véase Schulze-Motel, 1970).

A. brevipes Spruce $=$ A. rupestris (véase Bartram, 1964).

A. subnervis Hook. f. \& Wils. = A. nitida (véase Schulze-Motel, 1970).

Anisothecium jamesonii Mitt. $=$ A. hookeri.

Anomobryum prostratum (C.Müll.) Besch. $=$ A. filiforme .

Anomodon angustatus (Tayl.) Hampe $=$ Leskea.

Aongstroemia acerosa Hampe $=$ Anisothecium vaginatum .

A. bogotensis Hampe = Dicranella .

A. callosa Hampe = Dicranella.

A. consimilis Hampe $=$ Dicranella .

A. convoluta Hampe = Anisothecium .

A. crassinervis Hampe $=$ Dicranella heteromalla .

A. curviseta Hampe $=$ Campylopodium.

A. ditissima Hampe $=$ Dicranella .

A. muralis Hampe $=$ Microdus.

A. pusilla Hampe $=$ Microdus.

A. strumulosa Hampe = Dicranella.

A. vaginata (Hook.) C.Müll. = Anisothecium.

Apalodium pellucens (Hook.) Mitt. = Orthodontium.

Asteriscium decolorans (Hampe) Hilp. = Trichostomopsis australasiae (véase Robinson, 1970).

Atrichum bogotense Besch. $=$ A. androgynum (véase Nyholm, 1971).

Barbula affinis Hampe = Tortula pichinchensis.

B. apiculata Hampe $=$ B. replicata.

B. bogotensis Hampe $=$ Tortula.

B. decolorans Hampe = Trichostomopsis australasiae (véase Robinson, 1970).

B. fragilis (Tayl.) C.Müll. = Tortula.

B. goudotii Hampe = Tortula.

B. . lindigii Hampe = Gyroweisia .

B. trianae C.Müll. = Tortula fragilis.

Bartramia bogotensis Hampe $=B$. flavicans.

B. chrysea C.Müll. = Breutelia .

B. curvata Hampe $=$ Philonotis.

B. elegantula Tayl. = Philonotis (véase Robinson, 1967).

B. fontanella Hampe $=$ Philonotis.

B. gracilenta Hampe = Philonotis uncata var. gracilenta.

B. humilis Mitt. = Conostomum jamesonii.

$B$. jamesonii Tayl. = Leiomela bartramioides.

B. karsteniana C.Müll. = Breutelia.

B. lindigii (Hampe) Mitt. = Bartramidula.

B. lutescens Hampe $=$ Leiomela.

B. macrotheca Hampe = Breutelia tomentosa.

B. minuta Tayl. = Philonotis uncinata var. glaucescens (véase Florschütz, 1964).

B. pinnata Hampe = Philonotis scabrifolia.

B. squarrosa Mitt. = Breutelia.

B. striatula Mitt. $=$ Philonotis. 
B. subarcuata C.Müll. = Breutelia.

$B$. subpatens Hampe $=B$. flavicans.

B. subsessilis Tayl. = Anacolia laevisphaera.

B. tenella (Brid.) C.Müll. = Philonotis uncinata var. glaucescens (véase Florschütz, 1964).

B. tomentosa (Brid.) Web. \& Mohr = Breutelia.

B. trianae Hampe $=$ Breutelia.

B. versifolia Hampe = Philonotis uncinata var. glaucescens (véase Florschütz, 1964).

Bizotia densifolia (Thér.) Pier. = Campylopus pittieri (véase Florschütz, 1975).

Blindia inundata (Card.) Card. = B. magellanica var. inundata.

Brachymenium columbicum (DeNot.) Broth. = Osculatia columbica (véase Robinson, 1967).

B. papillosum Jaeg. = Pohlia.

Brachyodus flexisetus Hampe $=$ Brachydontium.

Brachysteleum lindigii Hampe = Ptychomitrium.

Breutelia sphagneticola Broth. \& Irmsch. = B. chrysea.

Bryoerythrophyllum angustulum (Herz.) Robins. = Trichostomopsis australasiae? (véase Robinson, 1975).

Bryum albicans Roehl. = Mniobryum wahlenbergii.

B. beyrichianum (Hornsch.) C.Müll. = Rhodobryum.

B. bimum (Schreb.) Turn. = B. pseudotriquetrum.

B. bulbillosum Mont. $=$ B. dichotomum.

B. clavicaule C.Müll. = Anomobryum.

$B$. corrugatum Hampe $=B$. argenteum.

B. goudotii Hampe = B. billardieri (véase Ochi, 1971).

B. grandifolium (Tayl.) C.Müll. = Rhodobryum.

B. julaceum Schrad. ex Gaertn., Meyer \& Scherb. = Anomobryum filiforme.

B. leptodontium Mitt. = Pohlia.

B. megalocarpum Hook. in Kunth = Acidodontium.

B. mniopsis C.Müll. = Rhodobryum.

B. papillosum C.Müll. = Pohlia.

B. paucifolium Hampe $=$ Pohlia.

B. prostratum C.Müll. = Anomobryum filiforme .

B. pycnopyxis C.Müll. = Rhodobryum.

B. rhamphostegium (Hampe) Mitt. = Acidodontium.

B. salaminae C.Müll. = Pohlia.

B. semi-ovatum Brid. = Anomobryum.

B. spectabile C.Müll. = Pohlia integridens (véase Robinson, 1967).

B. subcurvatum Mitt. = Pohlia.

B. truncorum (Brid.) Brid. = B. billardieri (véase Ochi, 1971).

B. turbinatum (Hedw.) Turn. var. minus Hook. in Kunth = Haplodontium jamesonii.

Calliergonella cuspidata (Hedw.) Loeske = Calliergon (véase Karczmarz, 1971).

Calymperes lindigii Hampe = Streptopogon calymperes.

Campylopus altissimus (C.Müll.) Jaeg. = C. jamesonii (véase Robinson, 1967).

C. friabilis (Hampe) Jaeg. =C. chrismarii.

C. penicillatus (Hornsch.) Jaeg. = C. lamellinervis.

C. polytrichoides DeNot. = C. pilifer Brid. (véase Gradstein \& Sipman, 1978).

C. porphyreodictyon (C.Müll.) Mitt. =C. filifolius (véase Frahm, 1975).

C. propinquus (Hampe) Mitt. = C. pauper (véase Robinson, 1967).

Catharinea abriaquiae C.Müll. = Polytrichadelphus.

C. aristata Hampe = Polytrichadelphus.

C. ciliata (Hook.f. \& Wils.) C.Müll. = Polytrichadelphus.

C. erosa Hampe = Oligotrichum.

C. polycarpa C.Müll. = Atrichum.

C. trianae (C.Müll.) Hampe = Polytrichadelphus longisetus (véase Robinson, 1967).

C. trichodon (Hook. f. \& Wils.) C.Müll. = Notoligotrichum (véase Smith, 1971).

Cladodium rhamphostegium Hampe = Acidodontium.

C. socorrense Hampe $=$ Bryum.

Conomitrium intromarginatum Hampe = Fissidens .

Cryphaea lindigii Hampe = Fissidens.

C. cuspidata Hampe $=C$. lindigiana.

C. gardneri Mitt. = Acrocryphaea. 
C. tenuissima Hook.f. \& Wils. = Barbella.

Cryptopodium hookeri Hampe = Leiomela bartramioides.

Cyclodictyon riparium (Mitt.) O.Kuntze $=C$. roridum.

Cynodontium bogotense Hampe $=$ Ditrichum.

Cynontodium rufescens (Hampe) Mitt. = Ditrichum.

C. strictum (Web. \& Mohr) Mitt. = Ditrichum rufescens.

Daltonia binervis Hampe $=$ Helicoblepharum daltoniaceum.

D. compressa Mitt. $=D$. bilimbata.

$D$. irrorata Mitt. $=D$. lindigiana.

D. ocanniana C.Müll. = D. gracilis.

D. wallisii C.Müll. = D. gracilis.

Dicranella crassinervis (Hampe) Jaeg. = D. heteromalla.

D. curviseta (Hampe) Mitt. = Campylopodium.

D. densa (Hook.) Mitt. = Microdus.

D. lindigiana (Hampe) Mitt. = Microdus.

D. macrocarpa Broth. \& Irmsch. = Microdus.

D. muralis (Hampe) Mitt. = Microdus.

D. pusilla (Hampe) Mitt. = Microdus.

D. vaginata (Hook.) Card. = Anisothecium.

Dicranodontium longisetum (Hook.) Williams = Atractylocarpus.

D. pusillum Thér. = Atractylocarpus longisetus.

Dicranum altissimum C.Müll. = Campylopus jamesonii (véase Robinson, 1967).

D. argyrocaulon C.Müll. = Campylopus.

D. chionophilum C.Müll. = Campylopus.

D. chrysodictyon Hampe = Campylopus.

D. concolor Hook. = Campylopus.

D. densum Hook. = Microdus.

D. filicaule Hampe = Campylopus.

D. flaccidissimum C.Mü-l. = D. bolivianum (fo. flaccidissimum véase Herzog, 1916).

D. jamesonii Hook. = Campylopus.

D. lamprodictyon Hampe = Campylopus.

D. longisetum Hook. = Atractylocarpus.

D. macrodon Hook. = Leucoloma.

D. pauperum Hampe = Campylopus.

D. porphyreodictyon C.Müll. = Campylopus filifolius (véase Frahm, 1975).

D. propinquum Hampe = Campylopus pauper (véase Robinson, 1967).

D. reflexisetum C.Müll. = Campylopus.

D. rosulatum Hampe = Campylopus.

D. sclerocarpum Hook.f. \& Wils. = Dicranella perrottetii.

D. speciosum Hook.f. \& Wils. = Chorisodontium.

D. spiripes C.Müll. = Atractylocarpus (véase Hermann, 1976).

D. strictulum C.Müll. = Campylopus (véase Robinson, 1967).

D. subconcolor Hampe = Campylopus.

D. wallisii C.Müll. = Chorisodontium.

Didymodon gracilis Hook. = Pilopogon.

Dissodon scabrisetus (Hook.) Grev. \& Arnott = Tayloria.

Distichophyllum pusillum Mitt. = Leskeodon.

Drepanophyllum facifolium (Schwaegr.) Wijk \& Marg. = Phyllodrepanium (véase Crosby 1970a).

Ectropothecium amabile Mitt. = Hypnum.

E. amphibolum Spruce ex Mitt. = Vesicularia.

E. apiculatum Mitt. = ? E. globitheca.

E. laevifolium Mitt. = Glossadelphus.

Entodon consanguineus (Hampe) Mitt. = Erythrodontium.

E. longisetus (Hook.) Mitt. = Erythrodontium.

E. lutescens Hampe $=E$. jamesonii.

E. onustus (Hampe) Mitt. = Mesonodon.

E. polycarpus Hampe $=$ E. jamesonii.

E. squarrosus (Hampe) Mitt. = Erythrodontium.

Entosthodon jamesonii (Tayl.) Mitt. = Funaria. 
E. lindigii (Hampe) Mitt. = Funaria.

E. lutescens (Hampe) Mitt. = Funaria.

E. pseudo-marginatus (Hampe) Mitt. = Funaria trumpffi.

E. undulatus (Hampe) Mitt. = Funaria.

Erythrophyllum campylocarpum (C.Müll.) Hilp. = Bryoerythrophyllum.

Eucatagonium politum (Hook. f. \& Wils.) Broth. = Catagonium.

Fissidens flexinervis Mitt. = F. prionodes fo. flexinervis (véase Florschütz, 1964).

$F$. tequendamensis Mitt. = F. lindigii.

$F$. tortilis Hampe \& C.Müll. = F. repandus.

Funaria calvescens Schwaegr. =F. hygrometrica var. calvescens.

Glyphocarpa laevisphaera Tayl. = Anacolia.

G. lindigii Hampe = Bartramidula.

G. strumosa Hampe = Bartramia.

G. taylorii Hampe = Anacolia laevisphaera.

Glyphomitrium lindigii (Hampe) Mitt. = Ptychomitrium.

G. lobuliferum Mitt. = Ptychomitrium.

Grimmia contermina C.Müll. = Racomitrium crispulum.

G. crispipila (Tayl.) C.Müll. = Racomitrium crispulum.

G. cucullatifolia (Hampe) Mitt. = Racomitrium.

G. lanuginosa (Hedw.) C.Müll. = Racomitrium.

G. ovata Web. \& Mohr =G. ovalis.

Groutiella apiculata (Hook.) Crum \& Steere = G. mucronifolia (véase Crosby, 1970b).

G. fragilis (Jaeg. \& Sauerb.) Crum \& Steere = G. tomentosa (véase Vitt \& Crum, 1970).

Guembelia bogotensis Hampe = Grimmia.

Gymnostomum bonplandii Hook. = Funaria.

Haplodontium jamesonii (Tayl.) Hampe $=H$. megalocarpum.

Harrisonia humboldtii (Hook.) Spreng. = Rhacocarpus purpurascens.

H. rhabdocarpa Hampe = Hedwigidium integrifolium.

Hedwigia humboldtii (Hook.) Hook. = Rhacocarpus purpurascens.

H. imberbis (Sm.) Spruce $=$ Hedwigidium integrifolium.

H. subrevoluta (C.Müll.) Mitt. = H. ciliata.

Heterophyllium affine (Mitt.) Fleisch. =H. nemorosum (véase Robinson \& Reed, 1966).

Hookeria albicans (Hedw.) Hook. \& Grev. = Cyclodictyon.

$H$. bogotensis Hampe = Adelothecium.

$H$. capillata Mitt. = Cyclodictyon.

H. crispa C.Müll. = Hookeriopsis.

H. cruegeriana C.Müll. = Hookeriopsis.

H. cuspidata C.Müll. = Cyclodictyon.

H. diversifolia Mitt. = Neohypnella.

H. falcata Hook. = Hookeriopsis.

H. gemmacea Mitt. = Amblytropis.

H. glandulifera Hampe $=$ Hookeriopsis.

H. hispidula Mitt. = Amblytropis.

H. humilis Mitt. = Cyclodictyon.

H. hypnacea C.Müll. = Hookeriopsis.

H. lindigiana Hampe = Cyclodictyon.

H. nivalis C.Müll. = Cyclodictyon.

H. ovata Mitt. = Amblytropis.

H. pallida Hornsch. $=$ Callicostella .

H. pilifera Hook. \& Wils. = Hypnella.

H. rorida Hampe $=$ Cyclodictyon.

H. rubriseta Mitt. = Cyclodictyon.

H. subfalcata Hampe = Hookeriopsis.

H. taylorii C.Müll. = Hookeriopsis.

H. variabilis Hornsch. ex Mitt. = Hookeriopsis.

H. velutina Hampe $=$ Hookeriopsis.

H. viridissima Mitt. $=$ Hookeriopsis.

Hookeriopsis killipii Williams = Thamniopsis.

H. pendula (Hook.) Jaeg. = Thamniopsis.

Hyophila lindigii Hampe = Gyroweisia. 
Hypnum acrophyllum Hampe = Leucomium.

H. affine Hook. in Kunth. = Heterophyllium nemorosum (véase Robinson \& Reed, 1966).

H. agnatum Hampe = Sematophyllum caespitosum.

H. andinum Hampe $=$ Plagiothecium.

H. anomalum Hampe = Lindigia aciculata.

H. apiculatum Hornsch. = ? Ectropothecium globitheca .

H. aquaticum Hampe = Platyhypnidium.

H. arzobispoae C.Müll. = Thuidium.

H. asperulum Hampe = Brachythecium.

H. aureo-sulfureum C.Müll. = Sematophyllum.

H. bonplandii Amott $=$ Homalothecium leskeoides.

H. caespitosum (Hedw.) Schrad. = Sematophyllum.

H. carantae C.Müll. = Thuidium.

H. chrysocladon Hampe = Brachythecium .

H. chrysophylloides Hampe $=$ Rhynchostegium.

H. chrysostegum C.Müll. = Sematophyllum.

H. clinocarpum Tayl. = Oxyrrhynchium remotifolium (Grev.) Broth. (véase Steere, 1948).

H. conchophyllum Tayl. = Rhynchostegium.

H. crassiusculum Brid. = Sematophyllum.

H. crithmifolium Hampe = Sematophyllum tequendamense .

H. cucullatifolium Hampe = Sematophyllum caespitosum.

$H$. delicatulum Hedw. = Thuidium recognitum var. delicatulum.

H. elatulum C.Müll. = Porothamnium.

H. exasperatum Hampe = Oxyrrhynchium praelongum.

H. fasciculatum Sw. ex Hedw. = Porothamnium.

H. flagelliferum Hampe = Porothamnium.

$H$. fragilirostrum Hampe $=$ Rhaphidorrhynchium.

H. frontinoae C.Müll. = Thuidium .

H. globitheca C.Müll. = Ectropothecium.

H. gratum P. Beauv. = Thuidium.

H. hispidulum Brid. = Campylium.

$H$. hospitans Hampe $=$ Isopterygium.

H. intermedium Mitt. ex Hampe = Thuidium arzobispoae.

H. kegelianum (C.Müll.) C. Müll. = Sematophyllum caespitosum.

H. laevifolium Mitt. = Brachythecium.

H. lancifrons Hampe = Porothamnium.

H. langsdorffii Hook. in Kunth $=$ Mittenothamnium.

H. lindigii Hampe = Rhaphidorrhynchium.

H. loriforme Hampe = Mittenothamnium.

H. loxense Hook. in Kunth = Sematophyllum caespitosum.

H. muricatulum Hampe = Thuidium minutulum.

H. neckeraeforme Hampe $=$ Porothamnium.

$H$. nigricans Hook. in Kunth = Squamidium.

H. novo-granatense Hampe = Plagiothecium.

H. occidentale Hampe $=$ Brachythecium.

H. patens Hook. = Zelometeorium (Véase Manuel, 1977a)

H. pauperum C.Müll. = Thuidium minutulum.

H. perspicuum Hampe $=$ Mittenothamnium diminutivum.

H. phyllogonium C.Müll. = Catagonium politum.

H. plumosum Hedw. = Brachythecium .

H. poeppigianum (Hampe) Hampe in C.Müll. = Vesicularia .

H. praelongum Hedw. = Oxyrrhynchium.

H. prasophyllum Hampe $=$ Brachythecium.

H. pseudo-delicatulum C.Müll. = Thuidium.

H. pseudo-lutescens Hampe $=$ Camptothecium.

H. pseudo-piliferum Hampe = Eurhynchium.

H. pseudo-protensum C.Müll. $=$ Thuidium.

H. pseudo-rutabulum Hampe = Brachythecium.

H. pygmaeum (Tayl.) C.Müll. = Isopterygium tenerum.

H. rugisetum Hampe $=$ Oxyrrhynchium. 
H. scariosum Tayl. = Rhynchostegium.

H. schlimii C.Müll. = Rhaphidorrhynchium.

H. schreberi Willd. ex Brid. = Pleurozium.

H. semi-tortum Mitt. = Eurhynchiella.

H. solutum Tayl. = Rigodium .

H. sparsiflorum Hampe = Porothamnium.

H. sparsum Hampe = Porothamnium sparsiflorum.

H. sticticola C.Müll. = Rhaphidorrhynchium.

H. subbrachycarpum Hampe = Sematophyllum.

H. subimponens Hampe $=H$. amabile.

H. subnitidum Hampe = Wijkia (véase Crum, 1971).

H. subobscurum Hampe = Mittenothamnium.

H. subrotundum Hampe $=$ Rhynchostegium.

H. subscabrum C.Müll. = Rhaphidorrhynchium.

H. tamariscinum Hedw. = Thuidium.

H. tequendamense Hampe = Sematophyllum.

H. thelistegum C.Müll. = Mittenothamnium diminutivum.

$H$. tocaremae Hampe $=$ Rhynchostegium.

H. toxarion Schwaegr. = Rigodium.

H. ulicon Tayl. = Rhynchostegium.

H. viscidulum Hampe = Mittenothamnium.

$H$. volvatum Hampe $=$ Mittenothamnium .

Hypopterygium scutellatum (Tayl.) C.Müll. $=H$. tamariscinum.

Illecebraria julacea (Hook.) Hampe = Aongstroemia.

Leiomela aristata Broth. $=$ L. aristifolia.

Lepidopilum daltoniaceum. Hampe = Helicoblepharum.

L. flexifolium Mitt. $=$ L. biductulosum.

L. hyalinum Hampe $=L$. angustifrons.

L. nutans Hampe $=$ Eriopus.

L. sprucei Mitt. $=$ Actinodontium.

L. subenerve Brid. $=$ L. scabrisetum.

Leptochlaena graciliseta Hampe = Mielichhoferia .

Leptodontium acutifolium Mitt. $=$ L. pungens (véase Zander, 1972).

L. filescens (Hampe) Mitt. $=$ L. flexifolium (véase Zander, 1972).

L. gracile C.Müll. ex Britt. = L. capituligerum (véase Zander, 1972).

L. gracilescens C.Müll. ex Britt. = L. capituligerum (véase Zander, 1972).

L. spinosum Williams = Zygodon pichinchensis (véase Zander, 1972).

L. subgracile Ren. \& Card. = L. longicaule (véase Zander, 1972).

L. subgrimmioides Broth. \& Thér. = L. pungens (véase Zander, 1972).

L. sulphureum (C.Müll.) Mitt. $=L$. viticulosoides var. panamense (véase Zander, 1972).

L. ulocalyx (C.Müll.) Mitt. = L. viticulosoides var. panamense (véase Zander, 1972).

L. vaginatum Herz. $=L$. wallisii (véase Zander, 1972).

Leptohymenium consanguineum Hampe $=$ Erythrodontium .

L. cylindricaule (C.Müll.) Hampe = Erythrodontium longisetum.

L. longisetum (Hook.) Hampe = Erythrodontium.

L. squarrosum Hampe = Erythrodontium.

Leptotrichum rufescens Hampe $=$ Ditrichum .

Lepyrodon suborthostichus (C.Müll.) Hampe $=$ L. tomentosus.

Leskea bonplandii Hook. = Homalothecium leskeoides.

L. gracillima (Tayl.) Mitt. = L. angustata.

L. involvens $\mathrm{Hedw}$. = Thuidium.

Leucobryum obtusifolium C.Müll. = Ochrobryum.

L. vulgare Hampe $=$ L. glaucum.

Lindigia curtipes Hampe $=L$. debilis.

L. hypnoides Hampe = L. aciculata.

L. imponderosa (Tayl.) Hampe = Papillaria.

L. pilotrichelloides Hampe = Papillaria imponderosa (véase Hampe, 1869).

Macromitrium apiculatum (Hook.) Schwaegr. = Groutiella mucronifolia (véase Crosby, 1970b).

$M$. contextum Hampe $=M$. punctatum.

$M$. didymodon Schwaegr. $=M$. richardii. 
M. frigidum C.Müll. = Macrocoma (véase Vitt, 1973).

$M$. liberum Mitt. $=M$. punctatum.

M. mucronifolium (Hook. \& Grev.) Schwaegr. = Groutiella.

$M$. ramosissimum Mitt. = Leptodontium viticulosoides var. panamense (véase Zander, 1972).

$M$. trianae C.Müll. $=M$. guatemalense.

$M$. undulatum Hampe = Leptodontium viticulosoides var. panamense (véase Zander, 1972).

Meteoriopsis consimilis (Hampe) Broth. = Meteoridium remotifolium (véase Manuel, 1977b).

M. patens (Hook.) Broth. = Zelometeorium (véase Manuel, 1977a).

M. patula (Hedw.) Broth. = Zelometeorium (véase Manuel, 1977a).

M. recurvifolia (Hornsch.) Broth. = Zelometeorium (véase Manuel, 1977a).

M. remotifolia (C.Müll.) Broth. = Meteoridium (véase Manuel, 1977b).

Meteorium appressum (C.Müll.) Mitt. = Papillaria nigrescens.

M. consimile (Hampe) Mitt. = Meteoridium remotifolium (véase Manuel, 1977b).

M. deppei (C.Müll.) Mitt. = Papillaria.

M. excavatum Mitt. = Orthostichidium guyanense (véase Florschütz, 1964).

M. filiferum (C.Müll.) Mitt. = Squamidium.

M. flexile (Hedw.) Mitt. = Pilotrichella.

M. fusco-viride (Hampe) Mitt. = M. illecebrum.

M. imponderosum (Tayl.) Mitt. = Papillaria.

M. leucotrichum (Tayl.) Mitt. = Squamidium.

M. longe-barbatum (Hampe) Mitt. = Squamidium leucotrichum.

$M$. nigrescens (Hedw.) Dozy \& Molk. = Papillaria.

M. nigricans (Hook.) Mitt. = Squamidium.

M. patens (Hook.) Mitt. = Zelometeorium (véase Manuel, 1977a).

M. remotifolium (C.Müll.) Mitt. = Meteoridium (véase Manuel, 1977b).

M. tenuissimum (Hook.f. \& Wils.) Mitt. = Barbella.

M. ternstroemiae (Brid.) Mitt. = Squamidium.

M. viride (C.Müll.) Mitt. = Pilotrichella.

Microthamnium andicola (Hook.) Mitt. = Mittenothamnium.

$M$. langsdorffi (Hook.) Mitt. = Mittenothamnium.

M. loriforme $($ Hampe) Mitt. $=$ Mittenothamnium.

M. reptans (Hedw.) Mitt. = Mittenothamnium.

M. substriatum Mitt. = Mittenothamniun.

M. thelistegum (C.Müll.) Mitt. = Mittenothamnium diminutivum.

M. tuerckheimii C.Müll. = Mittenothamnium reptans.

M. viscidulum (Hampe) Mitt. = Mittenothamnium.

$M$. volvatum (Hampe) Jaeg. = Mittenothamnium.

Mielichhoferia megalocarpa (Arnott) Mitt. = Haplodontium.

$M$. microstoma Hampe $=M$. pusilla.

Mniadelphus wallisii C.Müll. = Leskeodon.

Mnium lindigii Hampe = Rhizogonium.

M. polycarpum C.Müll. = Rhizogonium mnioides .

M. rostratum Schrad. = Plagiomnium rhynchophorum (véase T. Koponen, 1979).

M. spiniforme (Hedw.) C. Müll. = Rhizogonium.

Neckera atro-lutea C.Müll. = Meteorium.

N. consimilis Hampe $=$ Meteoridium remotifolium (véase Manuel, 1977b).

$N$. cylindricaulis C.Müll. = Erythrodontium longisetum.

N. densa (Hedw.) Wils. = Prionodon.

N. disticha Hedw. $=$ Neckeropsis.

$N$. filifera (C.Müll.) C.Müll. = Squamidium.

N. flexilis (Hedw.) C.Müll. = Pilotrichella.

N. fusco-viridis Hampe $=$ Meteorium illecebrum.

N. longe-barbata Hampe = Squamidium leucotrichum.

$N$. longiseta Hook. = Erythrodontium.

N. luteo-virens Tayl. = Prionodon (véase Griffin, 1973).

N. moritzii Hampe $=$ Calyptothecium .

$N$. nigricans (Hook.) Nees $=$ Squamidium .

N. obtusifolia Tayl. $=$ Neckeradelphus.

$N$. pachygaster C.Müll. = Pilotrichella .

N. remotifolia (C.Müll.) Hornsch. ex C.Müll. = Meteoridium (véase Manuel, 1977b). 
N. rufescens C.Müll. = Squamidium leucotrichum.

N. substriata Hampe = Porothamnium.

N. ternstroemiae (Brid.) C.Müll. = Squamidium.

N. turgescens C.Müll. = Pilotrichella flexilis.

Neobryum costatum Williams = Osculatia columbica (véase Robinson, 1967).

Oreoweisia ligularis Mitt. $=O$. bogotensis .

Orthodontium confine Hampe $=O$. pellucens .

Orthostichidium pentagonum (Hampe \& Lor.) C.Müll. = O. guyanense (véase Florschütz, 1964).

Orthotrichum longirostre Hook. = Macromitrium.

$O$. recurvans Schimp. in C.Müll. $=O$. pycnophyllum.

O. rufescens Hampe $=$ Zygodon .

Oxyrrhynchium clinocarpum (Tayl.) Broth. $=$ O. remotifolium (Grev.) Broth. (véase Steere, 1948).

Papillaria appressa (C.Müll.) Ångstr. = P. nigrescens.

P. fusco-viridis (Hampe) Jaeg. = Meteorium illecebrum.

Paraleucobryum densifolium Thér. = Campylopus pittieri (véase Florschütz, 1975).

Peromnium daguense Besch. $=$ Brachymenium.

P. jamesonii (Tayl.) Jaeg. = Brachymenium speciosum.

Philonotis glaucescens (Hornsch.) Broth. $=P$. uncinata var. glaucescens (véase Florschütz, 1964).

P. tenella (Brid.) Jaeg. = P. uncinata var. glaucescens (véase Florschütz, 1964).

$P$. umbonata Kindb. $=P$. marchica.

Pilopogon muelleri (Hampe) Broth. = Campylopus richardii.

$P$. piliferus Hampe $=P$. laevis (véase Thériot, 1936).

Pilopogonella laevis (Tayl.) Bartr. = Pilopogon (véase Thériot, 1936).

Pilotrichella consimilis (Hampe) Jaeg. = Meteoridium remotifolium (véase Manuel, 1977b).

P. fusco-viridis Hampe $=$ Meteorium illecebrum.

P. longibarbata Hampe = Squamidium leucotrichum.

P. nigricans (Hook.) Besch. = Squamidium.

P. remotifolia (C.Müll.) Besch. = Meteoridium (véase Manuel, 1977b).

P. tenuissima (Hook.f. \& Wils.) Hampe = Barbella.

Pilotrichum asperifolium Mitt. = Callicosta (véase Crosby, 1978).

P. bipinnatum (Schwaegr.) Brid. = Callicosta (véase Crosby, 1978).

P. fendleri C.Müll. = Callicosta (véase Crosby, 1978).

P. lindigii Hampe $=$ Porotrichum.

P. lycopodium C.Müll. = Prionodon densus (véase Robinson, 1967).

$P$. macranthum (Dozy \& Molk.) Hampe. = Squamidium nigricans.

$P$. nitidum Hampe $=$ Porotrichodendron.

P. patens (Hook.) C.Müll. = Zelometeorium (véase Manuel, 1977a).

P. ramosissimum Mitt. = Callicosta (véase Crosby, 1978).

P. sticticola C.Müll. = ? Squamidium nigricans.

P. wallisii C.Müll. = Callicosta ramosissima (véase Crosby, 1969 \& 1978).

Plagiothecium pseudo-tenerum Broth. \& Kindb. = Isopterygium.

P. tenerum (Sw.) Kindb. = Isopterygium.

Pleuropus bonplandii Broth. = Homalothecium leskeoides.

Poecilophyllum procumbens Mitt. = Leucoloma.

Pogonatum alpiniforme (Card.) Bartr. = Polytrichastrum tenellum (véase Smith, 1975).

$P$. cucullatum (Hampe) Mitt. $=P$. oligodus.

P. subbifarium Mitt. $=$ P. semipellucidum (véase Robinson, 1967).

P. viride Mitt. $=$ P. semipellucidum (véase Robinson, 1967).

Pohlia cruegeri (Hampe) Andrews in Grout $=$ Bryum.

Polytrichadelphus ericoides Mitt. = Polytrichum (véase Smith, 1976).

$P$. trianae (C.Müll.) Mitt. = P. longisetus (véase Robinson, 1967).

Polytrichum andinum Hampe = Pogonatum.

$P$. antillarum Rich. in Brid. $=P$. juniperinum.

P. aristiflorum Mitt. $=P$. juniperinum.

P. ciliatum Hook.f. \& Wils. = Polytrichadelphus.

P. convolutum Hook. in Kunth = Pogonatum semipellucidum (véase Mitten, 1869).

$P$. cucullatum Hampe = Pogonatum oligodus.

P. equisetiforme C.Müll. = P. juniperinum. 
$P$. giganteum Hook. $=$ Polytrichadelphus.

$P$. jamesonii Tayl. = Pogonatum oligodus .

$P$. longisetum Hook. = Polytrichadelphus.

$P$. neglectum Hampe = Pogonatum.

P. psilopiloides C.Müll. = Pogonatum.

P. purpurascens Hampe = Pogonatum.

$P$. rhynchomitrium C.Müll. $=P$. juniperinum.

$P$. semi-pellucidum Hampe = Pogonatum.

$P$. subcontortum Hampe = Pogonatum.

$P$. thysanomitrium C.Müll. $=P$. juniperinum.

P. trianae C.Müll. = Polytrichadelphus longisetus (véase Robinson, 1967).

P. trichodon Hook.f. \& Wils. = Notoligotrichum (véase Smith, 1971).

$P$. varians Hampe $=$ Pogonatum.

Porotrichum decompositum (Brid.) Mitt. = Homaliodendron.

P. fasciculatum (Hedw.) Mitt. = Porothamnium.

P. flagelliferum (Hampe) Mitt. = Porothamnium.

P. lancifrons $($ Hampe) Mitt. = Porothamnium.

$P$. neckeraeforme (Hampe) Mitt. $=$ Porothamnium.

P. nitidum (Hampe) Mitt. = Porotrichodendron.

P. sparsiflorum (Hampe) Mitt. $=$ Porothamnium.

P. stolonaceum Hampe $=$ Porotrichodendron superbum.

P. substriatum (Hampe) Mitt. = Porothamnium.

P. superbum (Tayl.) Hampe $=$ Porotrichodendron.

P. variabile Hampe $=P$. mutabile.

Potamium pulchellum (Hook.) Mitt. = Pterogonidium.

Prionodon divaricatus Mitt. $=\boldsymbol{P}$. fusco-lutescens (véase Robinson, 1967).

$P$. flagellaris Hampe $=P$. luteo-virens (véase Griffin, 1973).

$P$. laeviusculus Mitt. $=P$. luteo-virens (véase Griffin, 1973).

$P$. muelleri Hampe $=P$. densus (véase Robinson, 1967).

$P$. undulatus Mitt. $=P$. densus (véase Robinson, 1967).

Psilopilum trichodon (Hook.f. \& Wils.) Mitt. = Notoligotrichum (véase Smith, 1971).

Pterigynandrum consanguineum (Hampe) Hampe = Erythrodontium.

$P$. cylindricaule C.Müll. in Polak. = Erythrodontium longisetum.

$P$. longisetum (Hook.) Hampe $=$ Erythrodontium.

P. squarrosum (Hampe) Hampe = Erythrodontium.

Pterobryon duplicatum (Schwaegr.) Mitt. = Calyptothecium.

P. lindigii (Hampe) Besch. = Porotrichum.

Pterogonium fulgens (Hedw.) Sw. = Phyllogonium.

P. pulchellum Hook. $=$ Pterogonidium.

Racomitrium conterminum (C.Müll.) Jaeg. = R. crispulum.

R. crispipilum (Tayl.) Jaeg. = R. crispulum.

Rhabdoweisia cyathicarpa (Mont.) Mitt. = Amphidium.

$\boldsymbol{R}$. lindigiana (Hampe) Mitt. $=R$. fugax.

Rhacocarpus humboldtii (Hook.) Lindb. = R. purpurascens.

Rhaphidostegium galipense (C.Müll.) Ren. \& Card. = Sematophyllum.

R. loxense (Hook.) Jaeg. = Sematophyllum caespitosum.

R. subloxense Kindb. = ? Sematophyllum caespitosum.

R. subsimplex (Hedw.) Besch. = Sematophyllum.

Rhynchostegium aquaticum Jaeg. = Platyhypnidium.

R. deplanatiforme Kindb. = Isopterygium.

R. pulchellum (Hedw.) H.Robins. = Eurhynchium.

R. remotifolium (Grev.) H.Robins. = Oxyrrhynchium.

$R$. riparioides (Hedw.) Card. in Tourret = Platyhypnidium.

R. semiscabrum (Bartr.) H.Robins. = Eurhynchium.

Schistomitrium obtusifolium (C.Müll.) C.Müll. = Ochrobryum.

Seligeria lindigiana Hampe $=$ Microdus .

Sematophyllum agnatum (Hampe) Mitt. $=S$. caespitosum.

S. crinitifolium (Hampe) Mitt. (err. pro S. crithmifolium) =S. tequendamense.

S. cucullatifolium (Hampe) Mitt. = S. caespitosum.

S. decumbens Wils. ex Mitt. = Rhaphidorrhynchium. 
S. erythropodium Mitt. = Rhaphidorrhynchium.

S. fluviale Mitt. = Trichosteleum.

S. fragilirostrum (Hampe) Mitt. = Rhaphidorrhynchium.

S. leptothecium Mitt. $=S$. caespitosum.

S. lindigii (Hampe) Mitt. = Rhaphidorrhynchium.

S. loxense (Hook.) Mitt. = S. caespitosum.

S. pungens (Hedw.) Mitt. = Acroporium.

S. roridum Mitt. = Rhaphidorrhynchium.

S. subscabrum (C.Müll.) Mitt. = Rhaphidorrhynchium.

Sphaerothecium comosum Hampe =S. phascoides.

Sphagnum acutifolium Schrad. = S. capillifolium (véase Crum, 1975).

S. andinum Hampe $=$ S. magellanicum.

S. apollinairei Par. \& Warnst. = S. sparsum.

S. capillaceum (Weiss) Schrank = S. capillifolium (véase Crum, 1975).

S. coryphaeum Warnst. $=S$. meridense.

S. cymbifolium (Ehrh.) Hedw. = S. palustre.

S. lehmannii Warnst. = S. cuspidatum.

S. limbatum Mitt. = S. meridense.

S. medium Limpr. = S. magellanicum.

S. nemoreum Scop. = S. capillifolium (véase Crum, 1975).

S. pulchricoma C.Müll. = S. recurvum.

S. wallisii C.Müll. = S. magellanicum.

Sporledera lindigiana Hampe = Bruchia.

S. subenervis Hampe = Bruchia.

Stereodon affinis Mitt. = Heterophyllium nemorosum (véase Robinson \& Reed, 1966).

S. amabilis (Mitt.) Broth. = Hypnum.

S. hamatus Mitt. = Hypnum.

S. spiripes (C.Müll.) Mitt. = Hypnum.

Stokesiella praelonga (Hedw.) H.Robins. = Oxyrrhynchium.

Streptopogon latifolius Mitt. = S. lindigii.

S. rigidus Mitt. ex Salm. = S. calymperes.

$S$. setiferus Mitt. $=S$. lindigii.

Syrrhopodon crispatus Hampe = Bryoerythrophyllum campylocarpum.

S. fragilis Hampe = Trichostomum schlimii (véase Reese, 1978).

S. inflexus Mitt. = S. gaudichaudii (véase Reese, 1978).

S. iridans Mitt. = S. leprieurii (véase Reese, 1978).

S. pusillus Mitt. = S. leprieurii (véase Florschütz, 1964).

Systegium lindigii Hampe = Astomum.

Tayloria jamesonii (Tayl.) C.Müll. = Brachymitrion (véase A. Koponen, 1977).

T. mayorii Broth. \& Irmsch. = Brachymitrion moritzianum (véase A. Koponen, 1977).

T. moritziana C.Müll. = Brachymitrion (véase A. Koponen, 1977).

Thuidium antillarum Besch. = T. urceolatum.

$T$. delicatulum (Hedw.) Mitt. = T. recognitum var. delicatulum.

Thysanomitrium muelleri Hampe = Campylopus richardii.

$T$. phascoides Hampe = Sphaerothecium.

T. richardii (Brid.) Schwaegr. = Campylopus.

Tortula apiculata Mitt. = Barbula replicata.

T. arcuata Mitt. = Bryoerythrophyllum.

$T$. decidua Mitt. $=T$. bogotensis.

$T$. decolorans (Hampe) Mitt. = Trichostomopsis australasiae (véase Robinson, 1970).

$T$. glaucescens (Hampe) Mitt. = Barbula.

T. longirostris (Hampe) Mitt. = Barbula.

T. novo-granatensis (Hampe) Mitt. = Barbula .

T. rectifolia (Tayl.) Mitt. = Barbula.

T. schlimii (C.Müll.) Mitt. = Trichostomum.

T. stenocarpa (Hampe) Mitt. = Barbula.

$T$. trianae (C.Müll.) Mitt. = T. fragilis.

Trichostomum campylocarpum C.Müll. = Bryoerythrophyllum.

T. filescens Hampe = Leptodontium flexifolium (véase Zander, 1972).

T. luteum (Tayl.) Hampe = Leptodontium. 
T. orthotrichoides C.Müll. $=$ Leptodontium.

T. subcirrhifolium C.Müll. = Leptodontium viticulosoides var. panamense (véase Zander, 1972).

T. syntrichioides C.Müll. = Leptodontium.

T. wallisii C.Müll. = Leptodontium.

Webera papillosa (Jaeg.) Herz. = Pohlia .

W. spectabilis (C.Müll.) Besch. = Pohlia integridens (véase Robinson, 1967).

Weissia bogotensis $=$ Oreoweisia. .

W. cucullata (Hampe) Mitt. = Pottia.

W. hampei Mitt. $=$ Astomum.

W. lindigiana Hampe = Rhabdoweisia fugax.

W. lindigii (Hampe) Mitt. = Gyroweisia.

W. subcaespitosa (Hampe) Mitt. = Barbula.

W. tortula (Schwaegr.) Mitt. = Hyophila.

$W$. viridula Hedw. ex Brid. $=W$. controversa.

Zygodon aureus C.Müll. $=\mathrm{Z}$. goudotii.

Z. ceratodontoides C.Müll. $=Z$. subdenticulatus (véase Florschütz, 1964).

Z. denticulatus Tayl. $=Z$. reinwardtii.

Z. linguiformis C.Müll. = Z. obtusifolius.

Z. moritzianus C.Müll. = Z. reinwardtii.

Z. reinwardtii (Hornsch.) Braun in B.S.G. var. subintegrifolius Malta $=$ Z. subdenticulatus (véase Florschütz, 1964).

\section{BibLIOGRAFIA}

ANDErson, L. E. \& V. S. Bryan. 1958. Systematics of the autoicous species of Ditrichum subg. Ditrichum. Brittonia 10: 121-137.

BARKLEY, F. A. 1949. Colectores de plantas de Colombia. Revista Fac. Nal. Agron. Medellin Colombia Vol. 9, 35: 190.

Bartram, E. B. 1953. Paramo mosses of Venezuela and Colombia, collected by A. H. G. Alston. THE BRYOLOGIST 56: 165-168.

- 1955. Mosses of the Ecuadorian Andes collected by P. R. Bell. Bull. Brit. Mus. (Nat. Hist.) Bot. 2: 51-64.

- 1964. Mosses of Cerro Antisana, Ecuadorian Andes. Rev. Bryol. Lichénol. 33: 1-14.

Bescherelle, E. 1894. Musci in Cryptogamae Centrali-Americanae in Guatemala, Costa-Rica, Colombia \& Ecuador. Bull. Herb. Boiss. 2: 389-401.

Bizot, M., R. B. Pierrot \& T. Pocs. 1974. Bizotia Pierrot, a synonym of Campylopus. Rev. Bryol. Lichénol. 41: 339-341.

Brotherus, V. F. 1924-25. Musci. Spezieller Teil in die natürlichen Pflanzenfamilien. Bnd. $10 \& 11$.

Crosby, M. R. 1968. Proposal for the conservation of the name Pilotrichum Brid., Musci. Taxon 17: 595-597.

. 1969. A revision of the tropical American Moss Genus Pilotrichum. THE BRYOLOGIST 72: $275-343$.

. 1970a. Some remarks on the genus Drepanophyllum Schwaegr. Rev. Bryol. Lichenol. 37: $345-353$. 611 .

- 1974. Toward a revised classification of the Hookeriaceae. Jour. Hattori Bot. Lab. 38: $129-141$

1975. Lectotypification of Schizomitrium B.S.G. Taxon 24: 353-355.

- 1978. New Combinations in Callicosta (Musci), the Correct Name for Pilotrichum. ThE BRYOLOGIST 81: 435-437.

CRuM, H. 1957. A contribution to the moss flora of Ecuador. Svensk Bot. Tidskr. 51: 197-206.

- 1971. Nomenclatural changes in the Musci. THE BRYOLOGIST 74: 165-174.

1972. A taxonomic account of the Erpodiaceae. Nova Hedwigia 23: 201-224.

. 1975. Comments on Sphagnum capillaceum. Contr. Univ. Michigan Herb. 11: 89-93.

- \& M. R. Crosby. 1974. A new Sphagnum from high altitude Costa Rica. Ann. Missouri Bot. Gard. 61: 904-906.

EwAN, J. 1948. A review of Purdieanthus and Lehmanniella, two endemic Colombian genera of Gentianaceae, and biographical notes on Purdie and Lehmann. Caldasia 5: 90-98. 
Florschütz, P. A. 1964. The mosses of Suriname I. Flora of Suriname Vol. VI.

\& J. Florschütz-de WAaRd. 1975. Bizotia Pierrot, a Synonym of Campylopus. Rev. Bryol. Lichénol. 41: 339-341.

Frahm, J. P. 1975. Conspectus der mittel- und südamerikanischen Campylopus-Arten. J. Cramer. Bryophytorum Bibliotheca Bnd. 5.

Gradstein, S. R. \& H. J. M. Sipman. 1978. Taxonomy and World Distribution of Campylopus introflexus and C. pilifer (=C. polytrichoides): a New Synthesis. THE BRYOLOGIST 81: 114121.

Gruffin, D. 1970. Notes on the Tropical Genus Prionodon. Rev. Bryol. Lichénol. 37: $653-656$. 258 .

1975. Additions to the moss flora of Venezuela. The BRYologist 78: 212-215.

1977a. Más adiciones a la flora musqueña de Venezuela. ThE BRYOLOGIST 80: 181-184.

. 1977b. Leiomela lopezii, sp. nov., con observaciones sobre unas especies gimnostomas del genero. Rev. Bryol. Lichénol. 43: 383-387.

, M. LÓPEz Figueiras \& L. RuIz-TERÁN. 1973. Additions to the moss flora of Venezuela from the state of Mérida. Phytologia 25: 107-112.

Hampe, E. 1847. Ein Referat über die Columbischen Moose, welche von Herrn Moritz gesammelt wurden. Linnaea 20: 65-98.

1862. Beitrag zu einer Moosflora von Neu-Granada. Flora 29: 449-458.

—. 1862a, 1863. Species novas muscorum ab Alexandro Lindigio in Nova Granda collectas. Linnaea 31: 518-532; 32: 127-164.

ㄴ. 1865a, 1865b, 1866. Musci in Prodromus Florae Novo-Granatensis (Triana et Planchon). Ann. Sci. Nat. Bot. V, 3: 337-376; 4: 324-378; 5: 301-342.

․ 1869. Allgemeine Bemerkungen und Verbesserungen zu der Synopsis Florae NovaeGranadae. Musci. Bot. Zeit. (Berlin) 27: 865-870.

Hegewald, P. \& E. 1975. Verzeichnis der Laubmoose von Peru nach Literaturangaben. Jour. Hattori Bot. Lab. 39: 39-66.

․ 1977. Eine Moossammlung aus Peru I. Nova Hedwigia Bnd. 28: 731-758.

Hermann, F. J. 1976. Recopilacion de los Musgos de Bolivia. The Bryologist 79: 125-171.

Herzog, Th. 1916. Die Bryophyten meiner zweiten Reise durch Bolivia. Bibl. Bot., Heft 87. 1934. Die Bryophyten der Andenreisen von C. Troll. Musci. Hedwigia 74: 97-114.

\section{2.}

1949. Miscellanea Bryologica I. Neotropica. Memoranda Soc. Fauna Fl. Fenn. 25: 43-

HoOKER, W. J. 1818-20. Musci Exotici I \& II. London.

Instituto Geografico “Agustin Codazzi." 1969. Atlas de Colombia, 2a ed. 1971. Diccionario Geografico de Colombia I \& II.

IRMSCHER, E. 1914. Beiträge zur Laubmoosflora von Columbien. Mém. Soc. Neuchatel Sci. Nat. 5: $994-1012$.

IwATSUKI, Z. \& A. J. ShARP. 1967. The bryogeographical relationships between Eastern Asia and North America I. Jour. Hattori Bot. Lab. 30: 152-170.

KarCzmarz, K. 1971. A monograph of the genus Calliergon (Sull.) Kindb. Monographiae Botanicae XXXIV, Warszawa.

KindBerg, N. C. 1901. Contributions à la flore de l'Amérique du Sud. Rev. Bryol. 28: 54-56.

KOPONEN, A. 1977. Tayloria subgen. Pseudotetraplodon, subgen. nov., and new combinations in Brachymitrion, Moseniella and Tayloria. Ann. Bot. Fennici 14: 193-196.

Koponen, T. 1972. Speciation on the Mniaceae. Jour. Hattori Bot. Lab. 35: 142-154.

1979. A synopsis of Mniaceae (Bryophyta, Musci.) I. South and Central American taxa. Jour. Hattori Bot. Lab 46: (en curso de publicación).

KuNTH, C. S. 1822. Synopsis Plantarum quas, in itinere ad plagam Aequinoctialem orbis novi, collegerunt Al. de Humboldt et Am. Bonpland I: 46-65. Paris.

LEWINSKY, J. 1976. On the systematic position of Amphidium Schimp. Lindbergia 3: $227-231$.

MAAss, W. S. G. 1966. Untersuchungen über die Taxonomie und Verbreitung von Sphagnum VI. Nova Hedwigia 12: 81-105.

Malta, N. 1926. Die Gattung Zygodon Hook. et Tayl. Acta Horti Bot. Univ. Latvian 1: 1-184.

MANUEL, M. G. 1977a. A monograph of the genus Zelometeorium Manuel gen. nov. Jour. Hattori Bot. Lab. 43: 107-126.

—. 1977b. The genus Meteoridium (C.Müll.) Manuel, stat. nov. Lindbergia 4: 45-55.

Margadant, W. D. 1959. Typification and conservation of generic names in Musci. Acta Bot. Neerl. 8: 274. 
MEIJER, W. 1952. The genus Orthodontium. Acta Bot. Neerl. 1: 3-80.

Messmer, L. W. \& T. C. FrYe. 1947. The Polytrichum juniperinum group between South America and the United States. THE Bryologist 50: 259-268.

MiLler, H. A. 1971. An overview of the Hookeriales. Phytologia 21: 243-252.

MrtTen, W. 1869. Musci Austro-Americani. Jour. Linn. Soc., Bot. 12: 1-659.

Mullen, D. \& T. C. Frye. 1947. Middle and South American species of Oligotrichum. The BRYOLOGIST 50: 67-79.

MüLlER, C. 1847. Beiträge zu einer Flora der Aequinoctial-Gegenden der neuen Welt. Columbische Laubmoose. Linnaea 19: 193-220.

- 1848. Uber die Laubmoose der, von den Herren Funck und Schlim in Columbien veranstalteten, käuflichen Sammlung des Herren J. Linden in Luxemburg. Bot. Zeit. (Berlin) 6: 761-768; 779-781.

1849. Synopsis Muscorum Frondosorum I. Berlin.

- 1852. Plantae Wagnerianae Columbicae. Musci frondosi. Linnaea 25: 744-745.

- 1857. Manipulus muscorum e Flora Novae Granadae. Bot. Zeit. (Berlin) 15: 577-583.

620 .

1874. Novitates Bryothecae Müllerianae 2. Musci Novo-Granatenses. Linnaea 38: 572

- 1875. Musci Novo-Granatenses nonnulli novi praesertim. Flora 58: 529-536; 545-555.

NyнOLM. E. 1971. Studies in the genus Atrichum P. Beauv. Lindbergia 1: 1-33.

OCHI, H. 1971. What is true Bryum truncorum. THE BRYOLOGIST 74: 503-506.

Ortiz, L. C. 1963. Carta Geográfico de la Corporación Autónoma Regional de la Sabana de Bogotá y de los valles de Ubate y Chiquinquirá. Rapa CAR 1: 100.000 .

Parus, E. G. 1906. Muscinées des Andes de la Nouvelle Grenade. Rev. Bryol. Lichénol. 33: 102-105.

Purseli, R. A. 1973. Un Censo de los Musgos de Venezuela. The Bryologist 76: 473-500; 559.

ReESE, W. D. 1961. The genus Calymperes in the Americas. The Bryologist 64: 89-140.

- 1974. Syrrhopodon quintasii in the Americas. THE BRYologist 77: 242-243.

1977. The genus Syrrhopodon in the Americas I. The Elimbate species. THE BRYOLOGIST 80: 2-31.

- 1978. The genus Syrrhopodon in the Americas II. The Limbate Species. THE BrYOLOGIST 81: 189-225.

RoBinson, H. 1967. Preliminary studies on the Bryophytes of Colombia. The Bryologist 70: $1-43$.

-1970. A revision of the moss genus Trichostomopsis. Phytologia 20: 184-191.

1975. The mosses of Juan Fernandez Islands. Smithsonian Contr. Bot. 27: 1-88.

- L. B. Holm-NiELSEN \& S. JEPPESEN. 1971. Mosses of Ecuador I. Lindbergia 1: 66-74.

- 1 \& B. LqJTNANT. 1977. Mosses of Ecuador II. Lindbergia 4: 105-116.

\& C. F. ReEd. 1966. The status of the Moss genus Heterophyllium. The BryologisT 69: 317-323.

SALMON, E. S. 1903. A monograph of the genus Streptopogon Wils. Ann. Bot. (London) 17: $107-150$.

SCHUlze-Motel, W. 1970. Monographie der Laubmoosgattung Andreaea. Willdenowia 6: 25110.

SMITH, G. L. 1971. A conspectus of the Genera of Polytrichaceae. Mem. N. Y. Bot. Gard. 21: 183.

- 1975. Neotropical Polytrichaceae I. THE BRYOLOGIST 78: 201-202.

- 1976. Neotropical Polytrichaceae IV. The Bryologist 79: 93-95.

SPRUCE, R. 1908. Notes of a Botanist on the Amazon \& Andes I. A. R. Wallace (ed.). London.

STEARN, W. T. 1968. Humboldt, Bonpland, Kunth and Tropical American Botany. Lehre.

STEERE, W. C. 1936. Mosses of the Allan Hancock expedition of 1934. Hancock Pacific expeditions 3 (1). Univ. S. Califormia Press.

-1948. Contribution to the Bryogeography of Ecuador. I. A review of the species of Musci previously reported. THE BRYOLOGIST 51: 65-167.

Thériot, I. 1906. Mousses récoltés aux environs de Bogotá (Colombie). Bull. Acad. Int. Géogr. Bot. 197/198: 78-80.

- 1928. Etude sur Campylopus concolor (Hook.) Mitt. et C. jamesonii (Hook.) Jaeg. Arch. Bot. (Paris) 2: 185-188.

ㄴ. 1936. Mousses de l'Equateur. Rev. Bryol. Lichénol. 9: 5-36.

- 1937. Additions á la flore bryologique de la Colombie. Rev. Bryol. Lichénol. 10: 11-18. 
1939. Complément á la flore bryologique de la Bolivie et de la Colombie. Rev. Bryol. Lichénol. 11: 40-66.

VitT, D. H. \& H. CRUM. 1970. Groutiella tomentosa new to the United States. The BryologisT 73: $145-149$.

1973. A revisionary study of the genus Macrocoma. Rev. Bryol. Lichenol. 39: 205-220.

WELCH, W. H. 1960. A monograph of the Fontinalaceae. Nijhoff, The Hague.

. 1966. The Hookeriaceae of Mexico. THE BRYOLOGIST 69: 1-68.

1969. The Hookeriaceae of Cuba. The Bryologist 72: 93-136.

1971. The Hookeriaceae of Jamaica, Hispaniola and Puerto Rico. The Bryolocist 74:

77-130.

1974. The Hookeriaceae of Central America. ThE BRYologist 77: 328-404.

1976. Hookeriaceae. N. Amer. Flora II, 9: 1-133.

WijK, R. van DER, W. D. Margadant \& P. A. Florschütz. 1959-69. Index Muscorum I-V. Regnum Veg. 17, 26, 33, 48, 65.

Williams, R. S. 1908. Mosses from tropical America. Bull. Torrey Bot. Club 34: 569-571.

1925. Some undescribed mosses from Colombia. THE BRYOLOGIST 28: 59-64.

1930. Colombian mosses, apparently undescribed. THE BRYOLOGIST 33: 73-82.

WILSON, W. 1847. Brief characters of some New Mosses, collected in New Granada by Mr. W. Purdie. London Jour. Bot. 6: 289-292.

ZANDER, R. H. 1968. Barbula inaequalifolia Tayl. new to North America. THE Bryologist 71: $41-44$.

. 1972. Revision of the Genus Leptodontium in the New World. THE Bryologist 75: 213-280.

. 1977a. Rhabdoweisia crenulata and Erythrophyllopsis andina from Colombia. THE BRYOLOGIST 80: 158-160.

1977b. The Tribe Pleuroweisieae (Pottiaceae) in Middle America. The Bryolocist 80: 233-269. 